Supporting Information

\title{
Triarylporphyrin meso-Oxy Radicals: Remarkable Chemical Stabilities and Oxidation to Oxophlorin $\pi$-Cations
}

Daiki Shimizu, ${ }^{\dagger}$ Juwon Oh, ${ }^{\ddagger}$ Ko Furukawa, ${ }^{\circledR *}$ Dongho Kim,${ }^{\ddagger *}$ and Atsuhiro Osuka ${ }^{\dagger *}$ ${ }^{\dagger}$ Department of Chemistry, Graduate School of Science, Kyoto University, Sakyo-ku, Kyoto 606-8502, Japan. ${ }^{\ddagger}$ Spectroscopy Laboratory for Functional $\pi$-Electronic Systems and Department of Chemistry, Yonsei University, Seoul 120-749, South Korea.

§Center for Instrumental Analysis, Niigata University, Nishi-ku, Niigata 950-2181, Japan.

\section{Contents}

1. NMR Spectra and NMR Experiments

2. ESR Spectra

3. Mass Spectra

4. Absorption and Emission Spectra

5. X-Ray Crystallographic Analysis

6. Electrochemical Analysis

7. Temperature Dependent Magnetic Susceptibility

8. Theoretical Calculations

9. Time-resolved TA Spectra and Fluorescence Decay

10. Supporting References

11. Optimized Molecular Geometries 


\section{NMR Spectra and NMR Experiments}
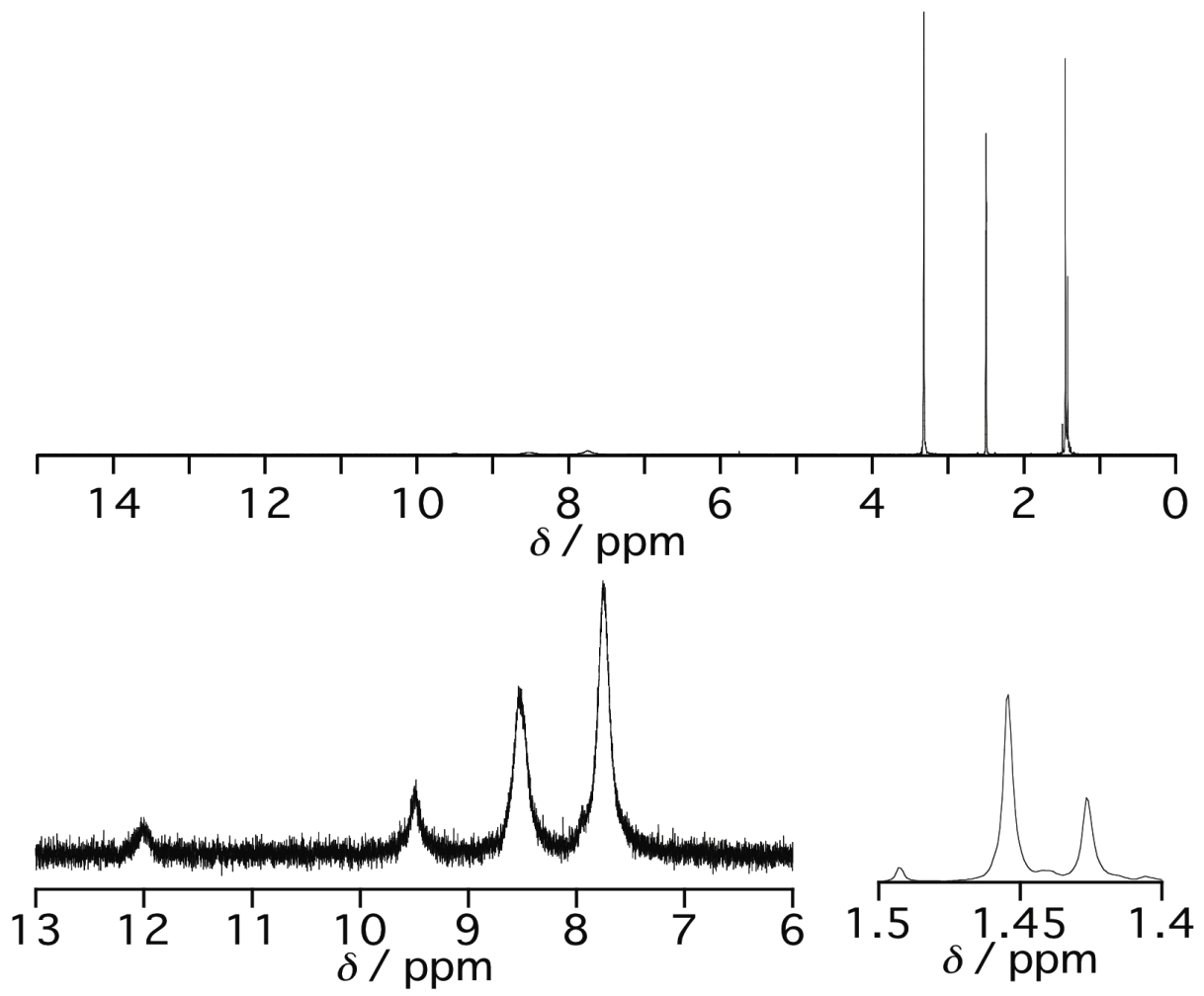

Figure S1-1. ${ }^{1} \mathrm{H}$ NMR spectrum of $7 \mathrm{Ni}$ in $\mathrm{DMSO}-d_{6}$ at room temperature.

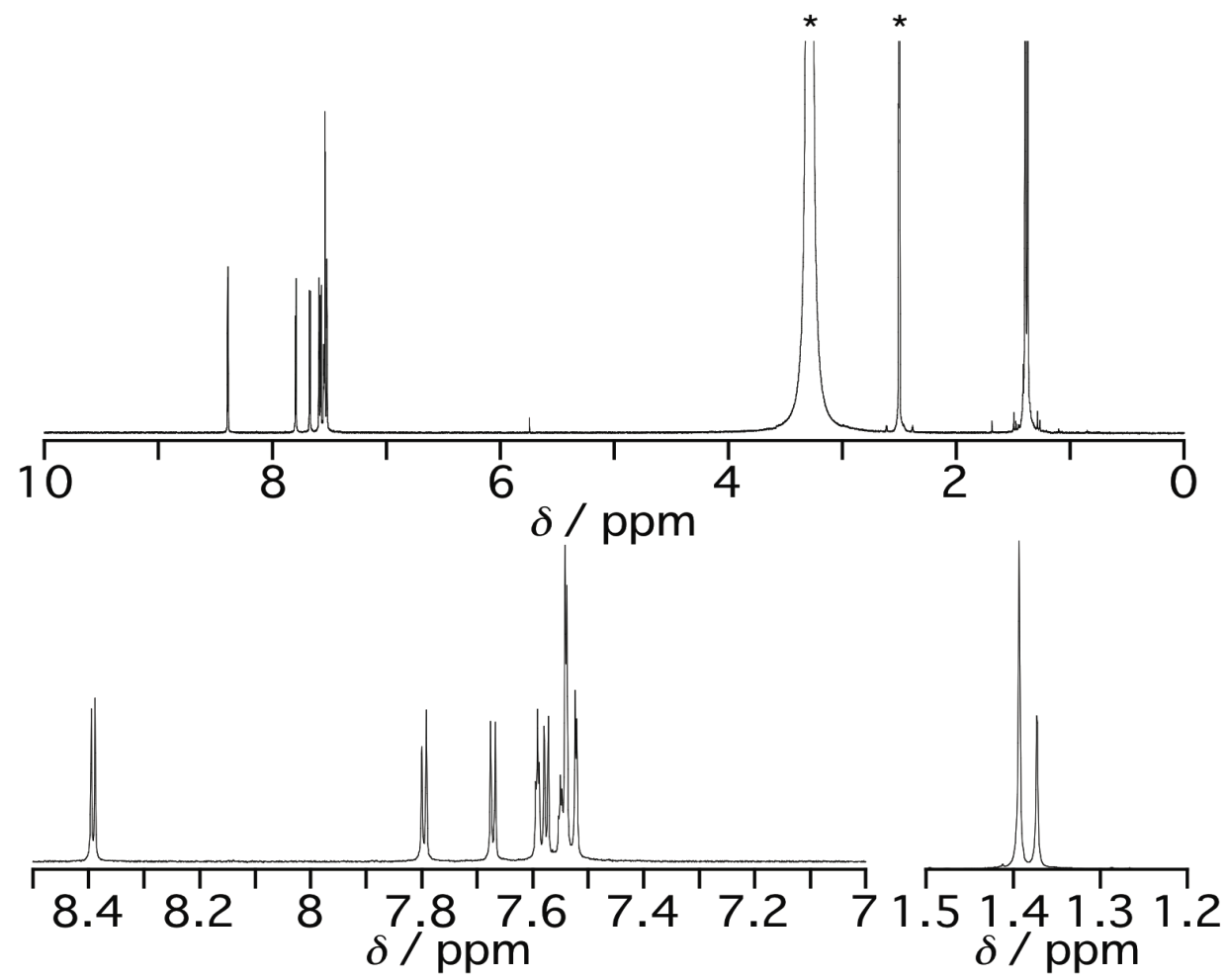

Figure S1-2. ${ }^{1} \mathrm{H}$ NMR spectrum of $7 \mathbf{N i}$ in $\mathrm{DMSO}-d_{6}$ with hydrazine monohydrate at room temperature ( ${ }^{*}$ indicates residual solvents and hydrazine). 


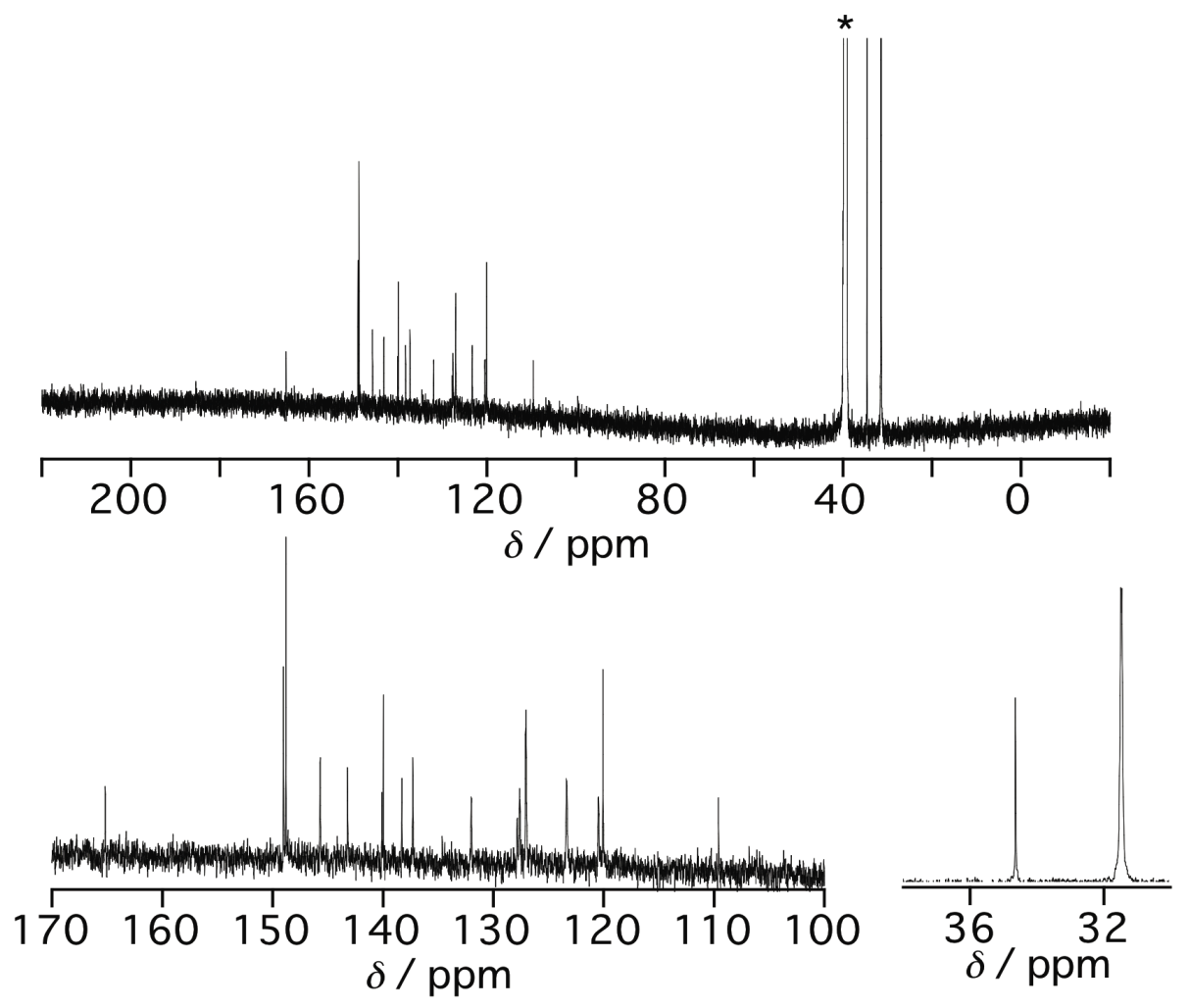

Figure S1-3. ${ }^{13} \mathrm{C}$ NMR spectrum of $7 \mathbf{N i}$ in DMSO- $d_{6}$ with hydrazine monohydrate at room temperature $(*$ indicates residual solvents).
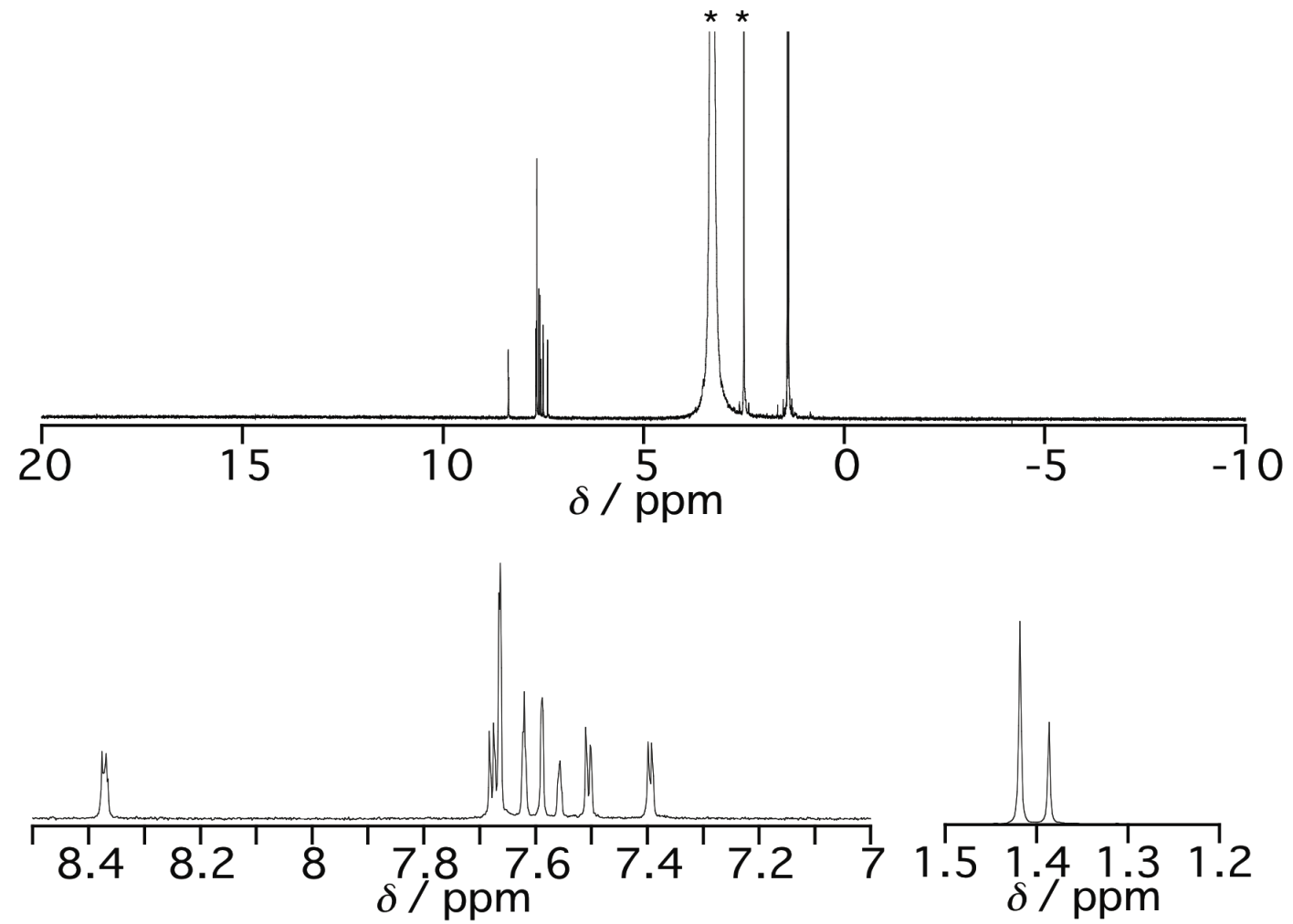

Figure S1-4. ${ }^{1} \mathrm{H}$ NMR spectrum of $7 \mathbf{H}_{2}$ in DMSO- $d_{6}$ with hydrazine monohydrate at room temperature $(*$ indicates residual solvents and hydrazine). 

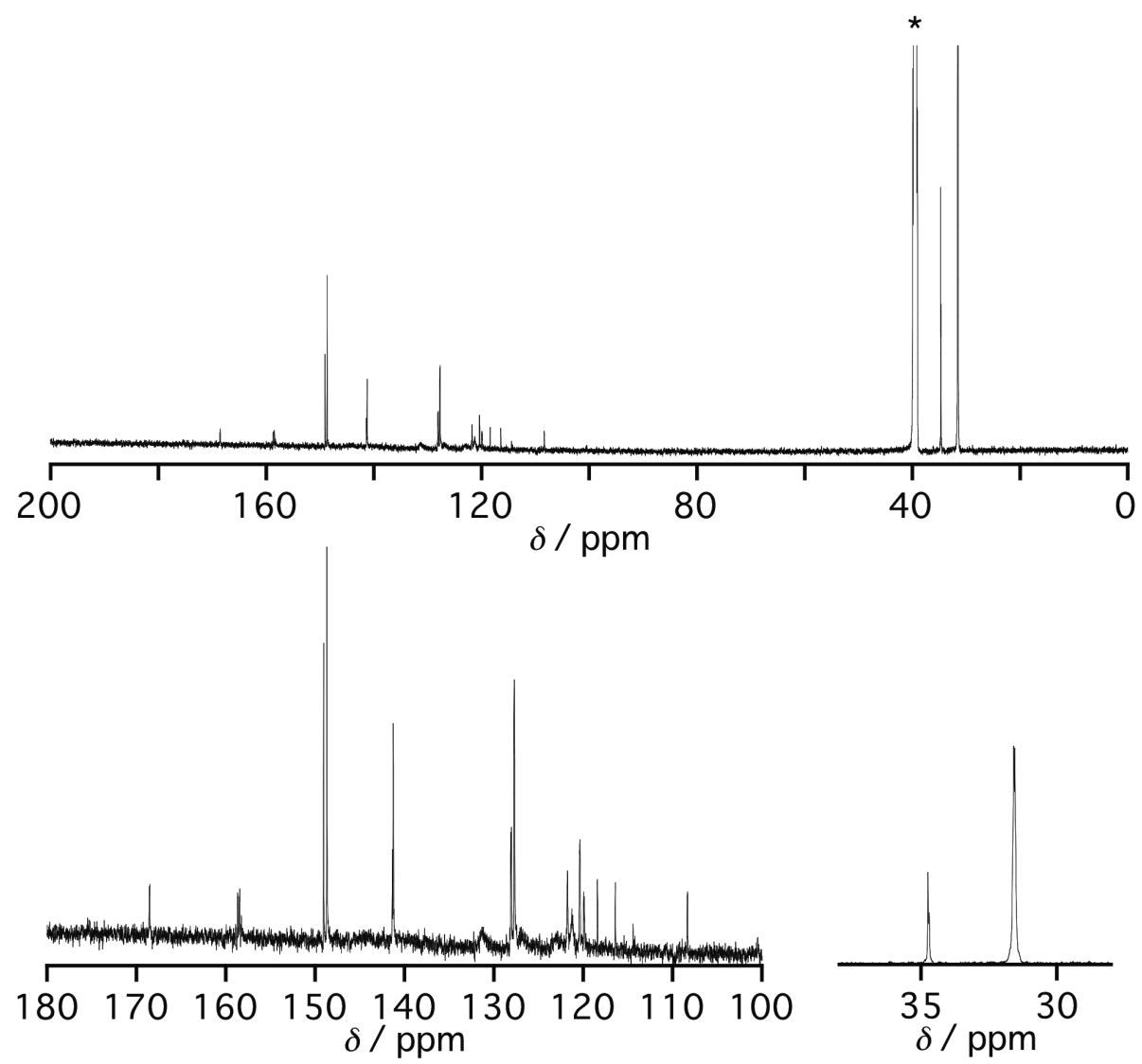

Figure S1-5. ${ }^{13} \mathrm{C}$ NMR spectrum of $\mathbf{7} \mathbf{H}_{\mathbf{2}}$ in DMSO with hydrazine monohydrate at room temperature (* indicates residual solvents).
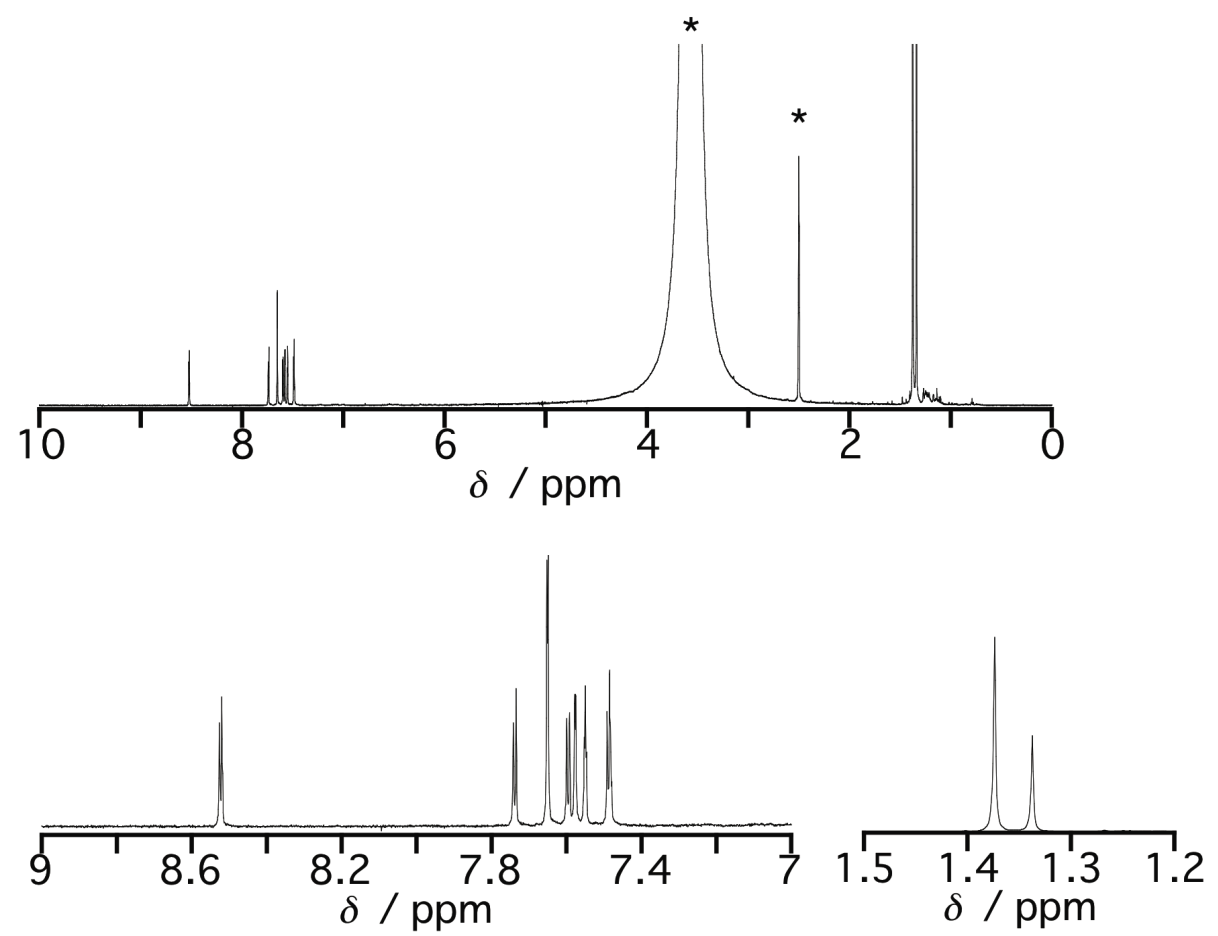

Figure S1-6. ${ }^{1} \mathrm{H}$ NMR spectrum of $7 \mathbf{Z n}$ in DMSO- $d_{6}$ with hydrazine monohydrate at room temperature (* indicates residual solvents and hydrazine). 


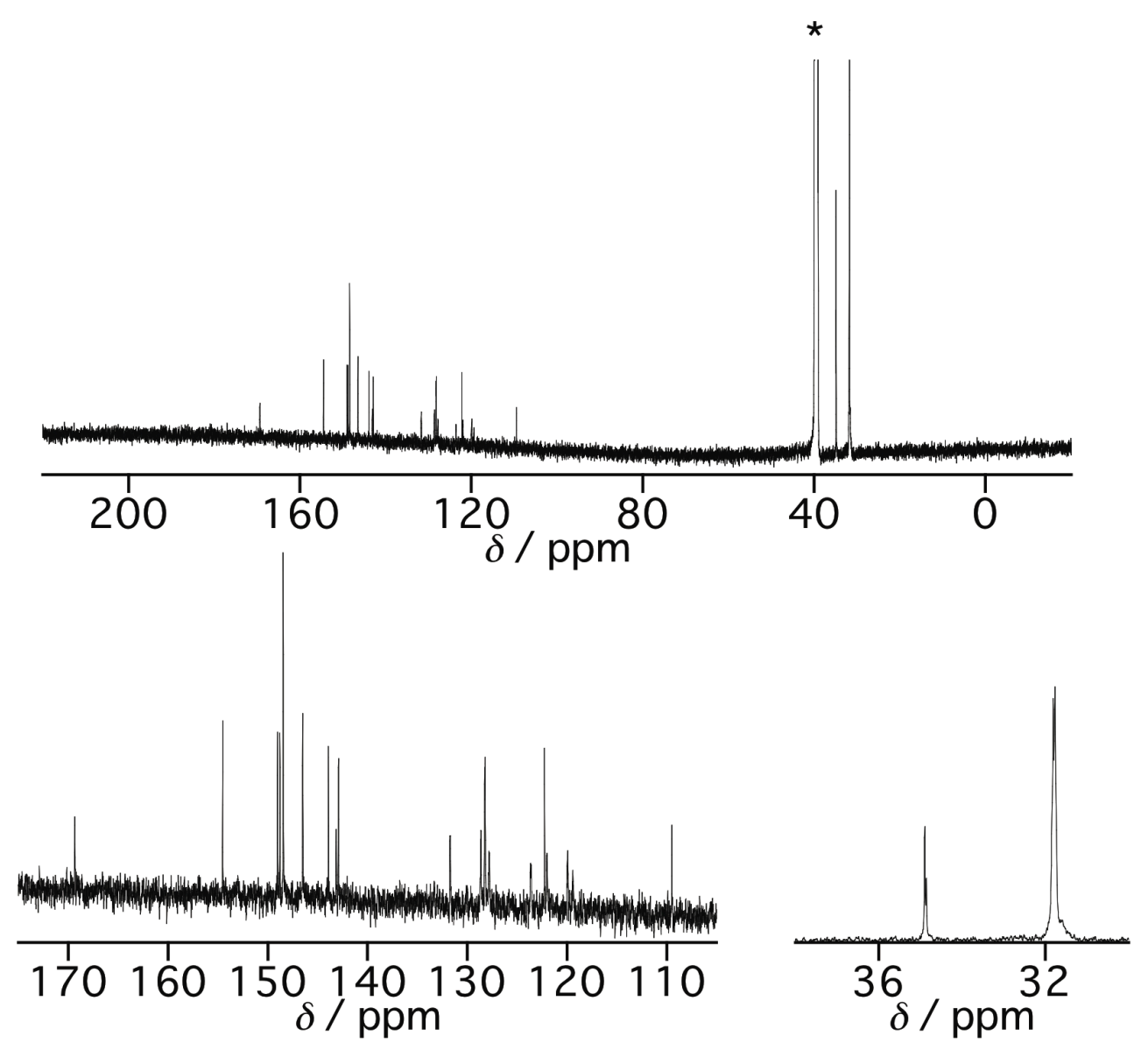

Figure S1-7. ${ }^{13 \mathrm{C}}$ NMR spectrum of $7 \mathbf{Z n}$ in DMSO- $d_{6}$ with hydrazine monohydrate at room temperature $(*$ indicates residual solvents).

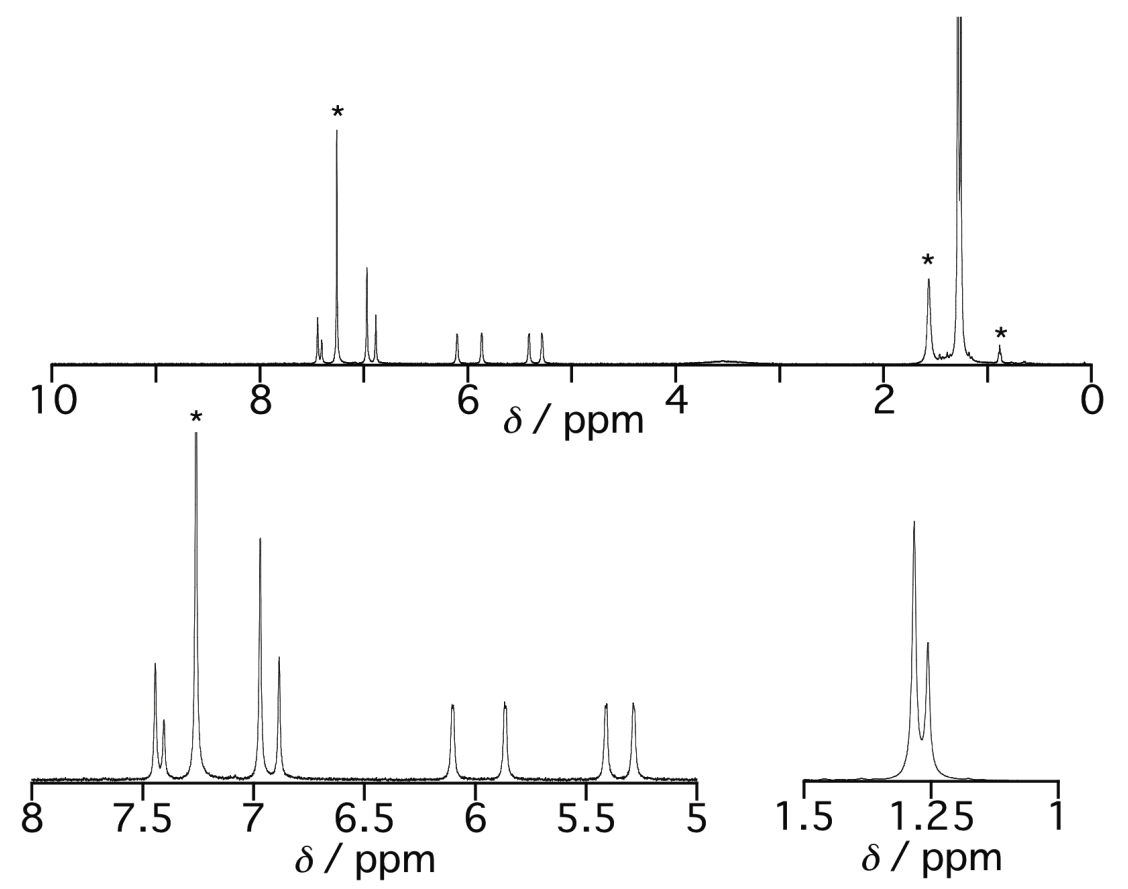

Figure S1-8. ${ }^{1} \mathrm{H}$ NMR spectrum of $\mathbf{1 0 N i}$ in $\mathrm{CDCl}_{3}$ at room temperature $(*$ indicates residual solvents and impurities). 

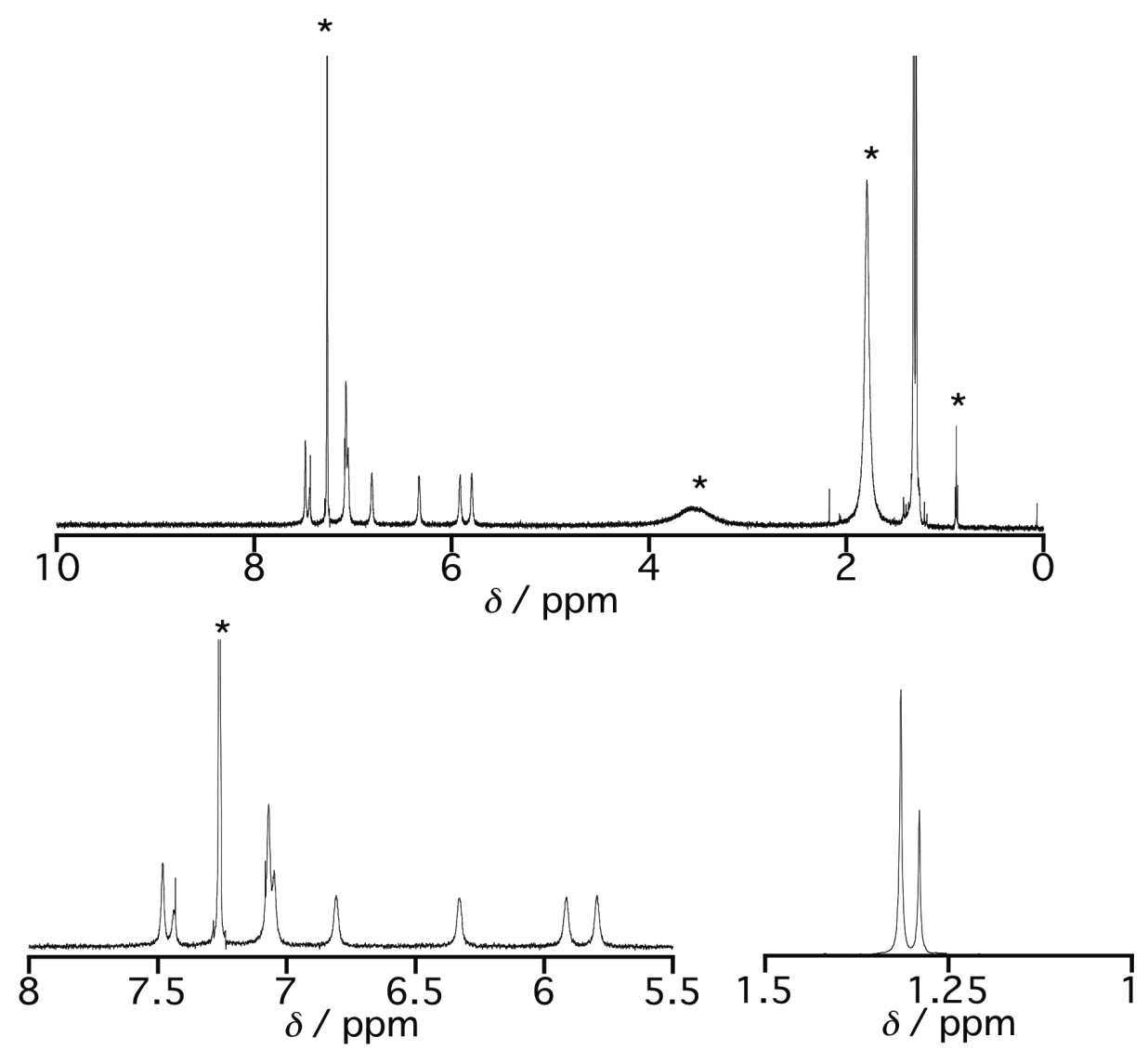

Figure S1-9. ${ }^{1} \mathrm{H}$ NMR spectrum of $\mathbf{1 0 Z n}$ in $\mathrm{CDCl}_{3}$ at room temperature $\left({ }^{*}\right.$ indicates residual solvents and impurities). 


\section{ESR Spectra}

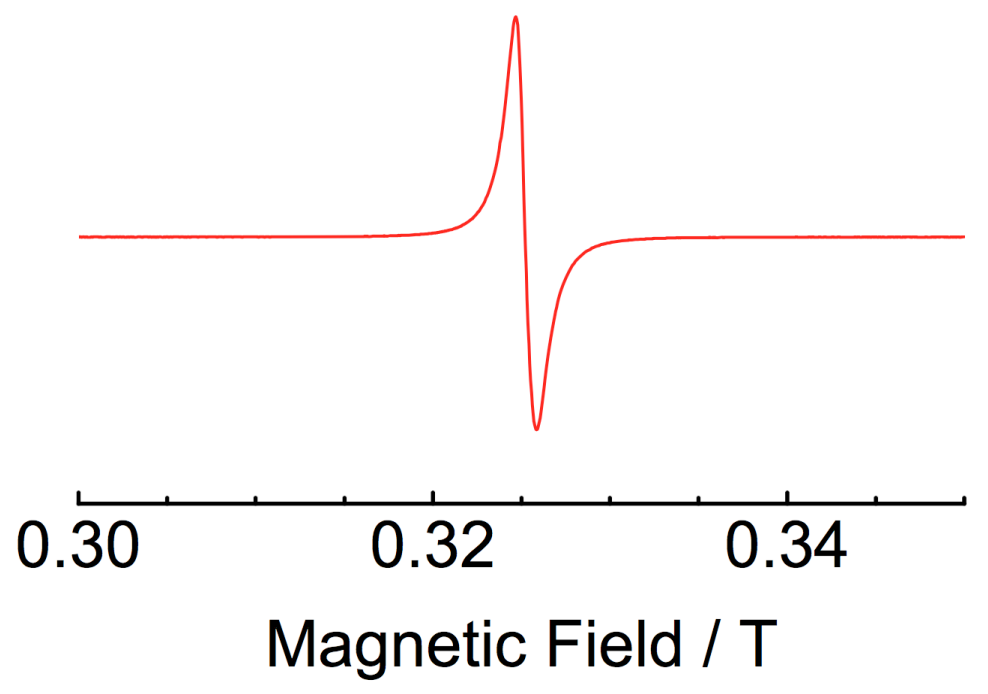

Figure S2-1. Observed ESR spectrum of $\mathbf{8 H}_{2}$ (1.0 $\mathrm{mM}$ in toluene) at at room temperature $(g=$ 2.0028).
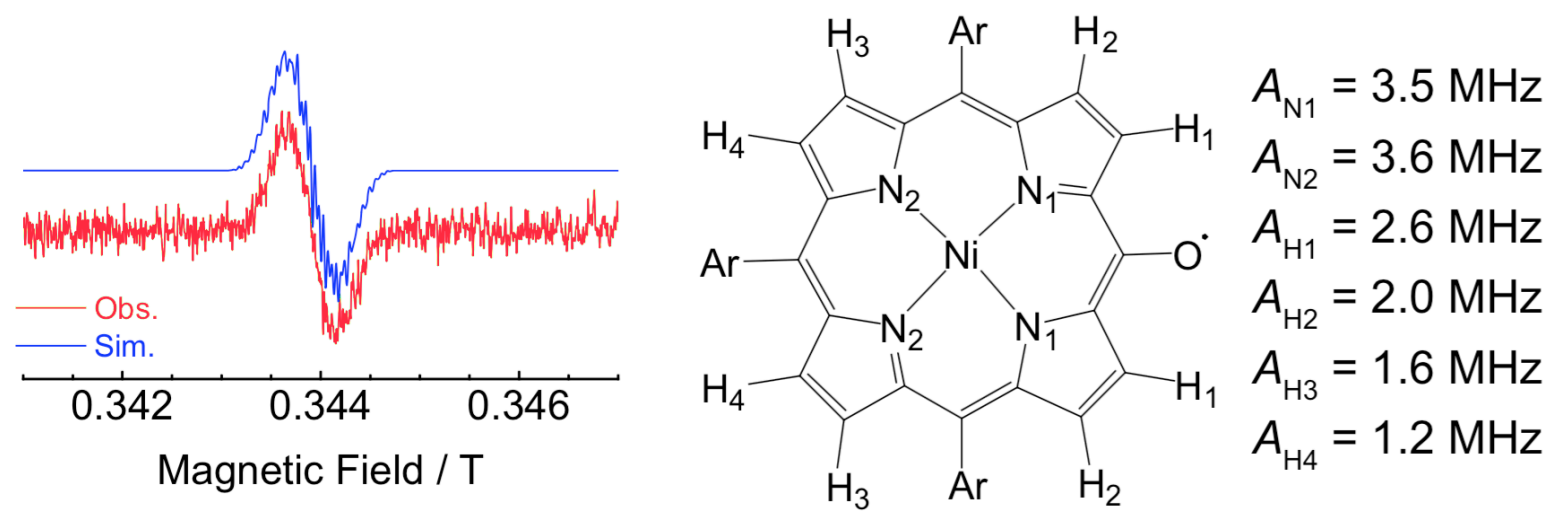

Figure S2-2. Observed and simulated ESR spectra of $\mathbf{8 N i}$ (o.10 mM in toluene) at room temperature $(g=2.0035)$.
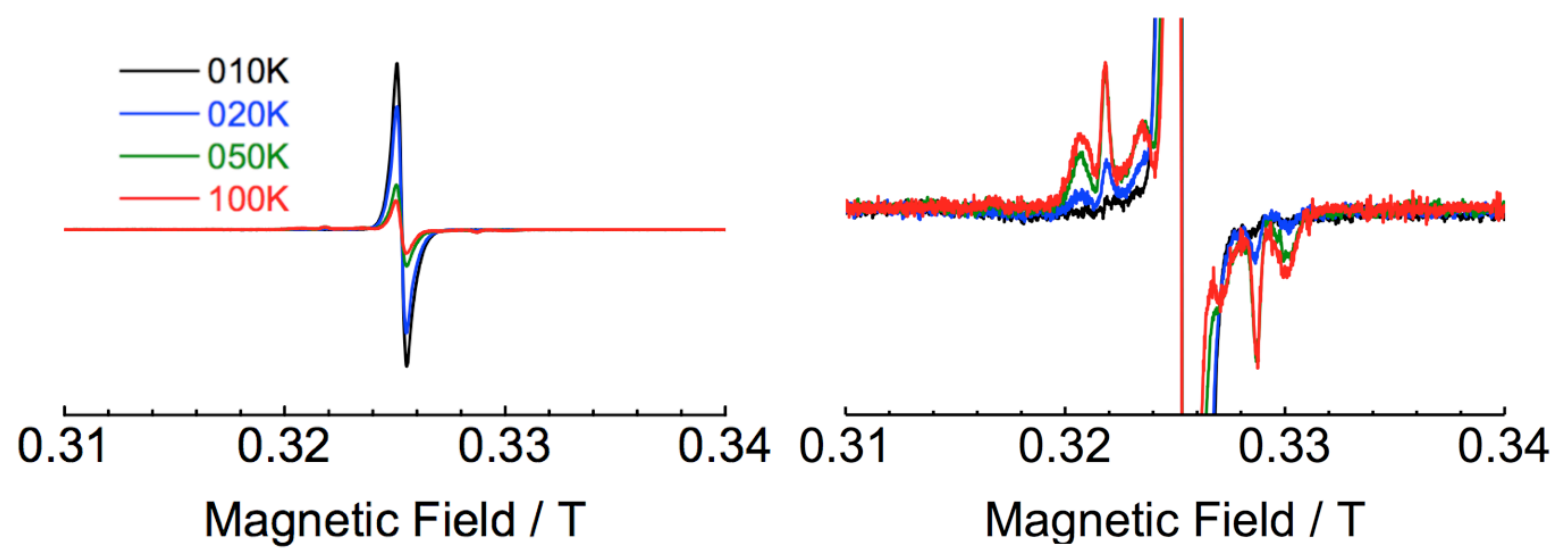

Figure S2-3. Observed VT-ESR spectra of $\mathbf{8 Z n}$ and $9 \mathrm{Zn}$ (1.15 $\mathrm{mM}$ based on monomer in toluene, $g$ $=2.0022$ ). 

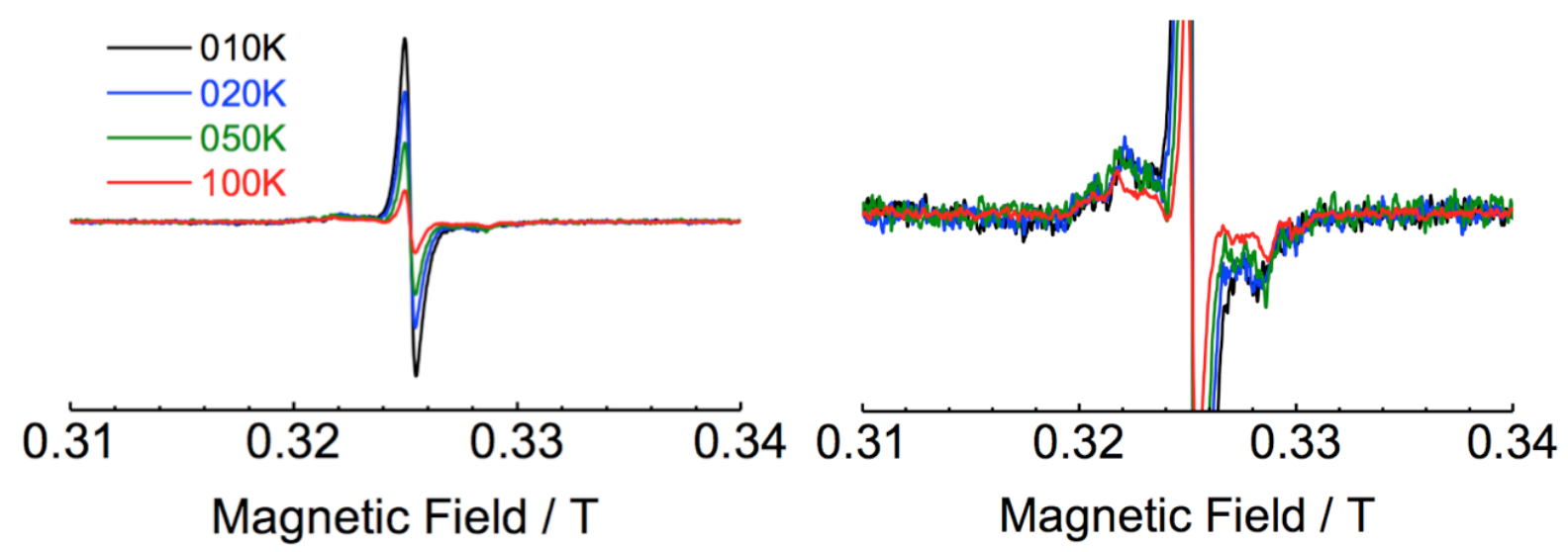

Figure S2-4. Observed VT-ESR spectra of $\mathbf{8 Z n}$ and $9 Z \mathbf{Z n}$ (o.115 mM based on monomer in toluene, $g$ $=2.0022$ ).

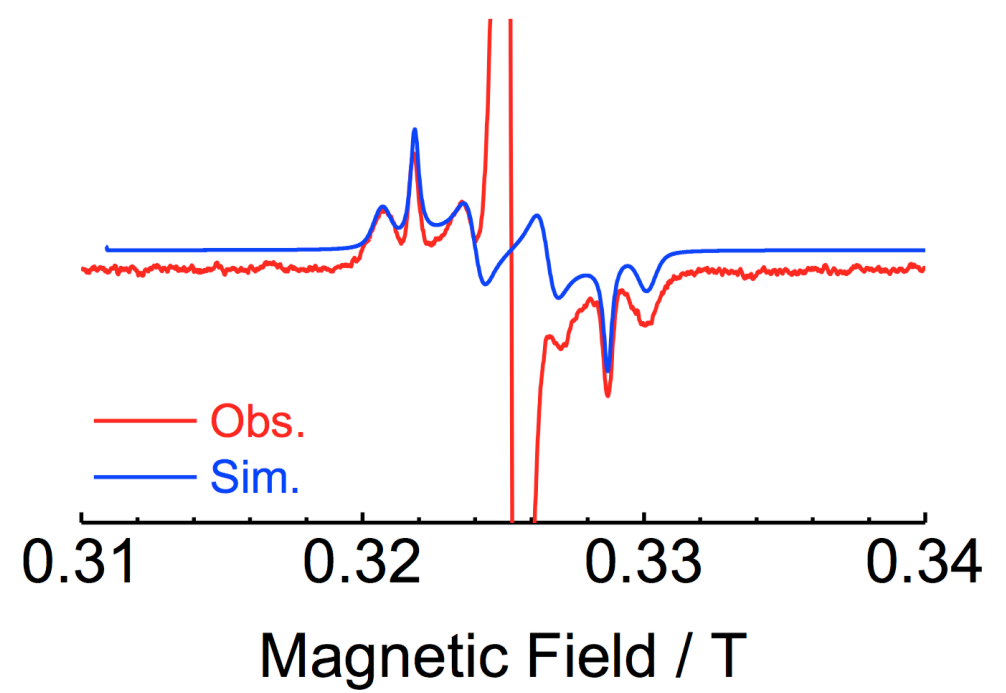

Figure S2-5. Comparison of the observed ESR spectrum of $\mathbf{8 Z n}$ and $9 \mathbf{Z n}$ at $100 \mathrm{~K}$ with the simulated ESR spectrum of $\mathbf{9 Z n}$ at $100 \mathrm{~K}$.
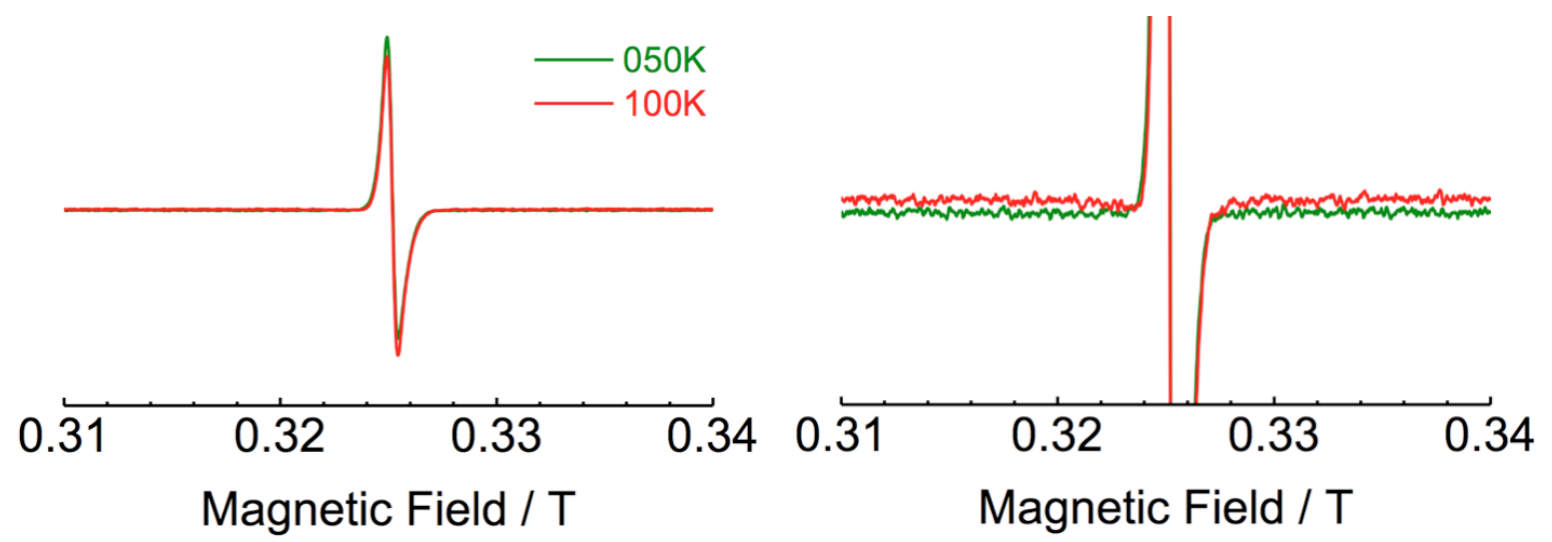

Figure S2-6. Observed VT-ESR spectra of 8Zn-py (o.115 mM in toluene/pyridine, $g=2.0020$ ). 


\section{Mass Spectra}

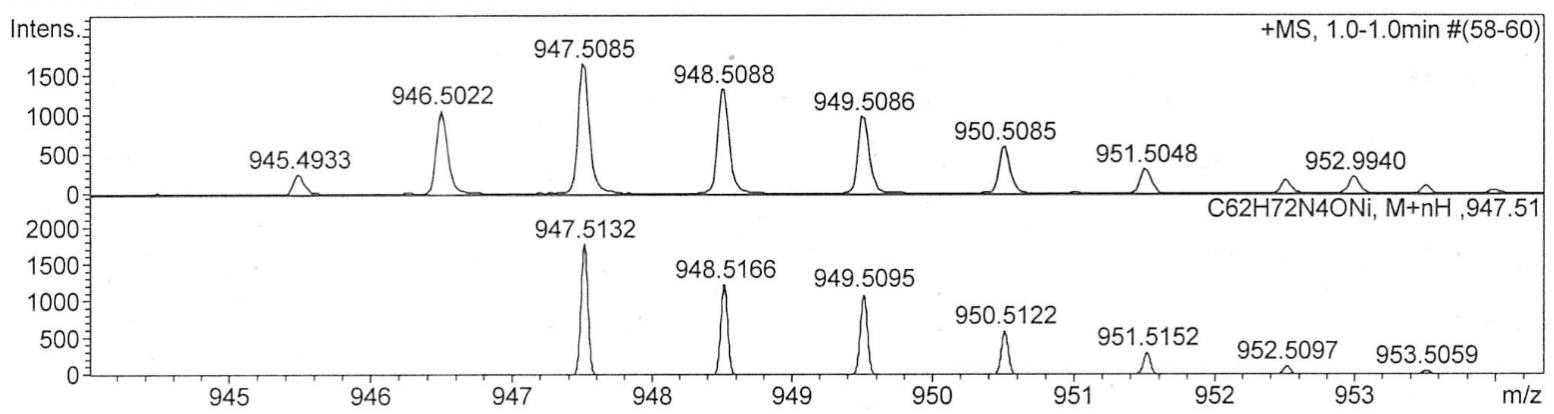

Figure S3-1. HR-APCI-TOF MS spectrum of $7 \mathbf{N i}$ (top: observed, bottom: simulated).

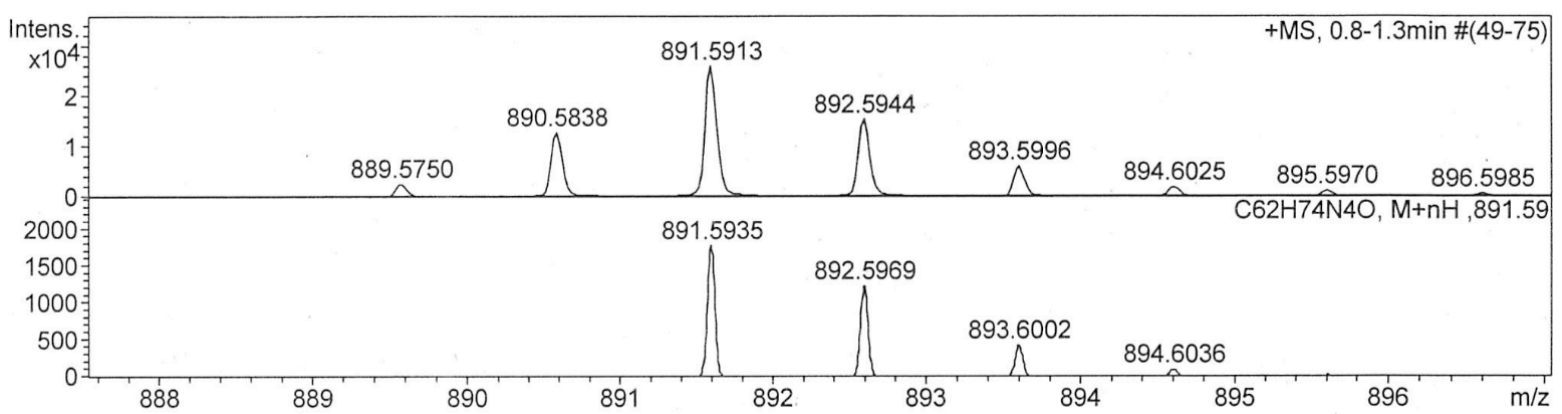

Figure S3-2. HR-APCI-TOF MS spectrum of $7 \mathbf{H}_{\mathbf{2}}$ (top: observed, bottom: simulated).

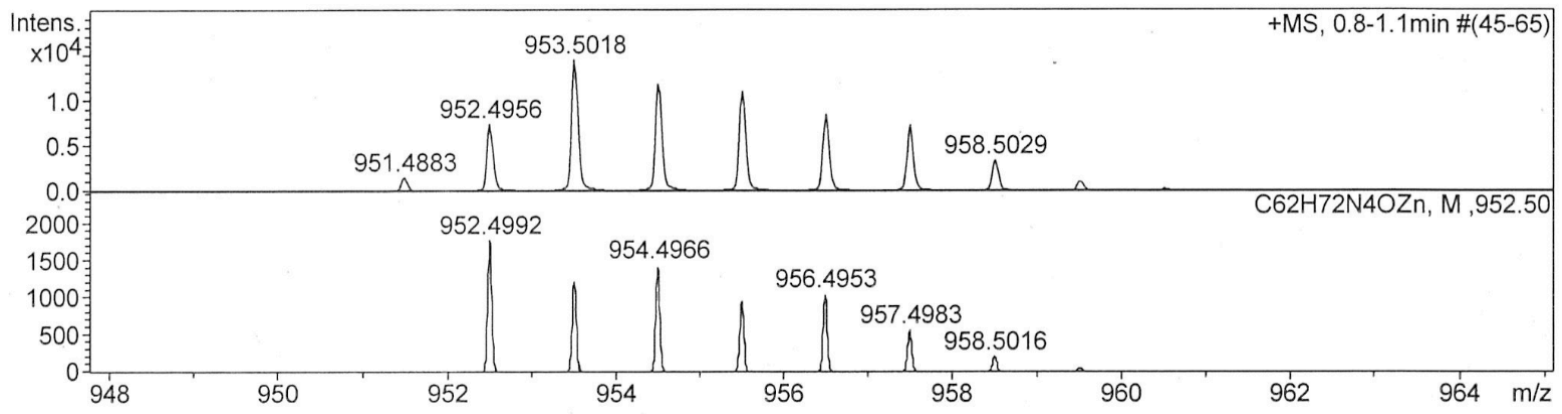

Figure S3-3. HR-APCI-TOF MS spectrum of 7Zn (top: observed, bottom: simulated).

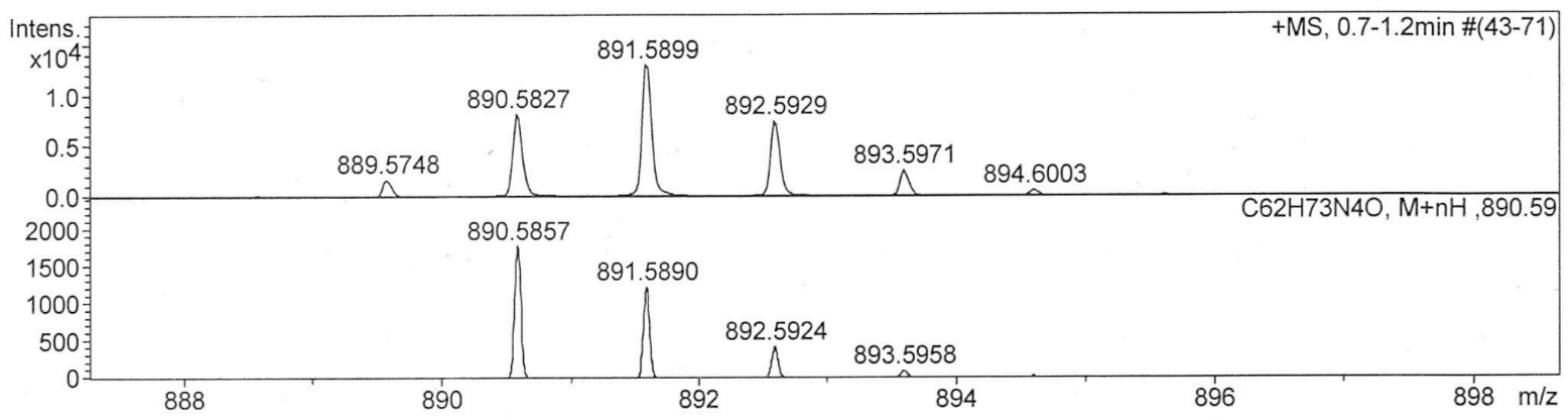

Figure S3-4. HR-APCI-TOF MS spectrum of $\mathbf{8} \mathbf{H}_{2}$ (top: observed, bottom: simulated). 


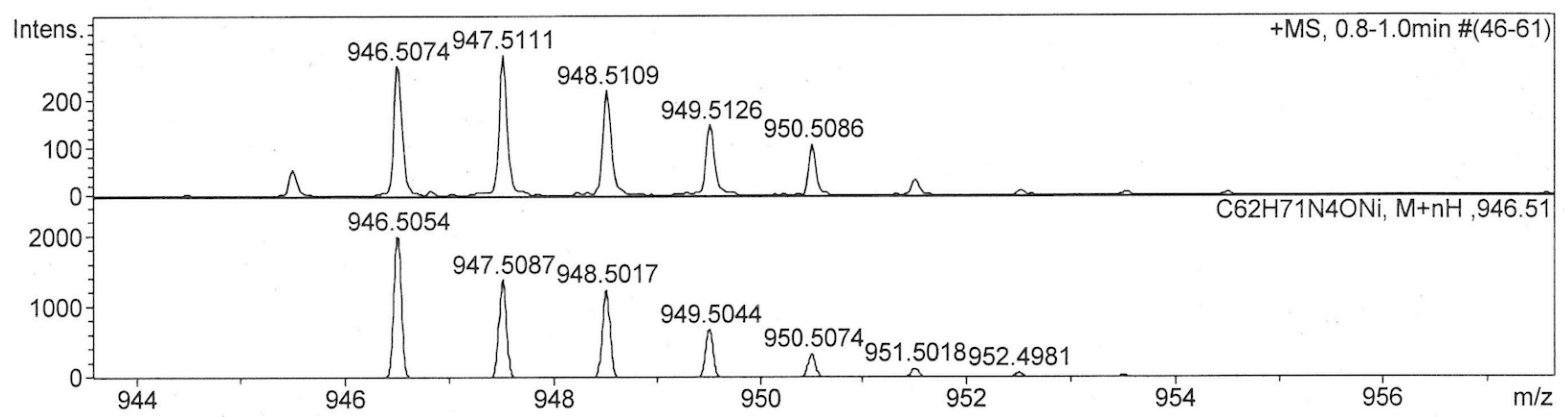

Figure S3-5. HR-APCI-TOF MS spectrum of 8Ni (top: observed, bottom: simulated).

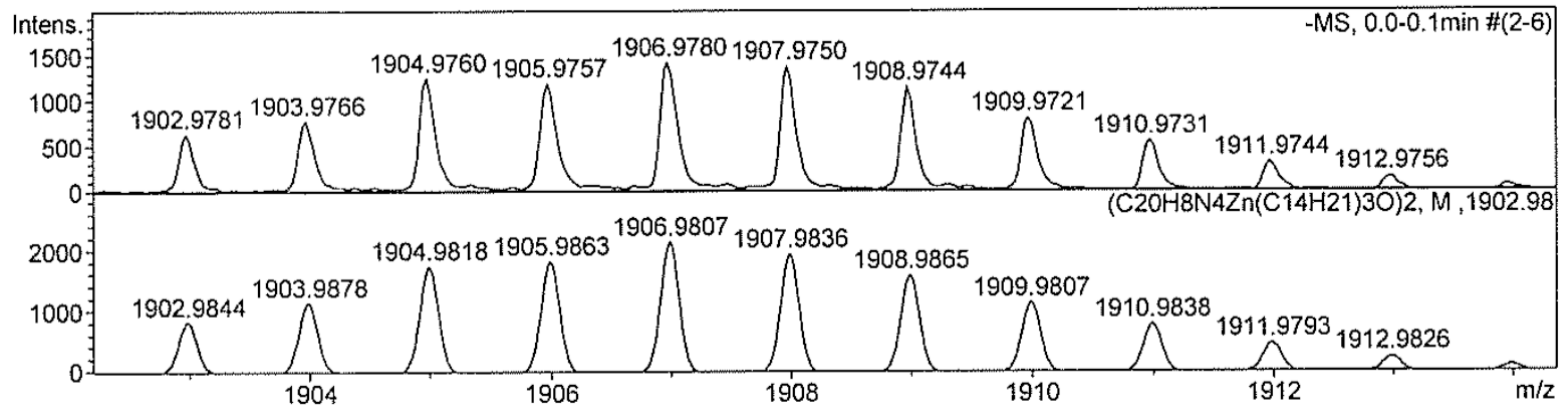

Figure S3-6. HR-ESI-TOF MS spectrum of $\mathbf{9 Z n}$ (top: observed, bottom: simulated).

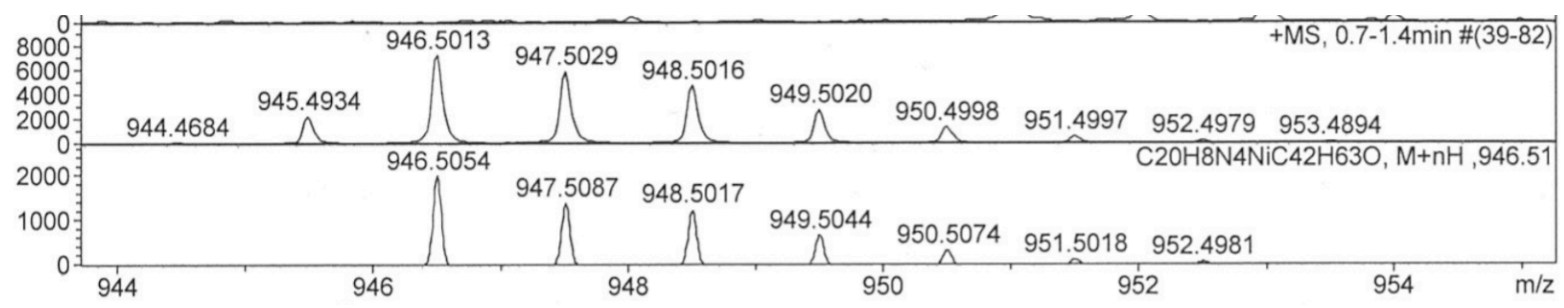

Figure S3-7. HR-APCI-TOF MS spectrum of 1oNi (top: observed, bottom: simulated).

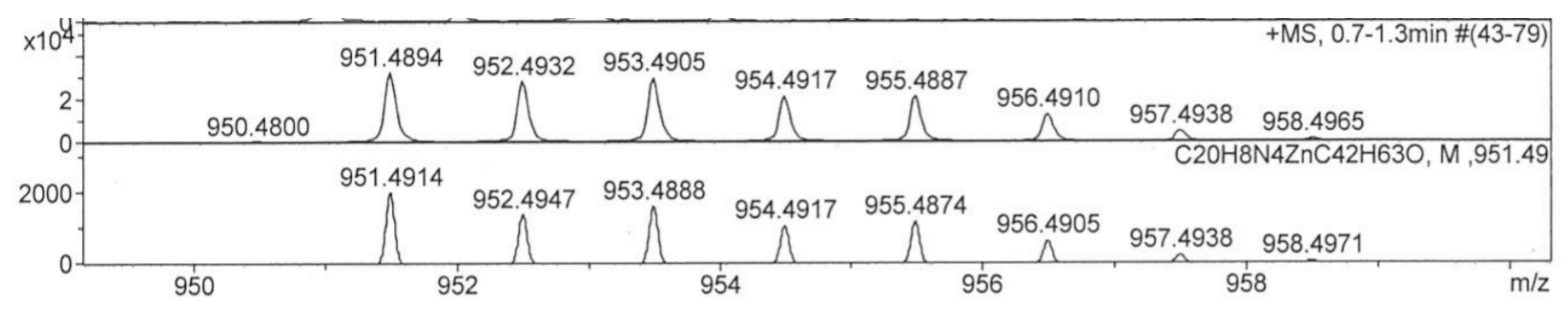

Figure S3-8. HR-APCI-TOF MS spectrum of 1oZn (top: observed, bottom: simulated). 


\section{Absorption and Emission Spectra}

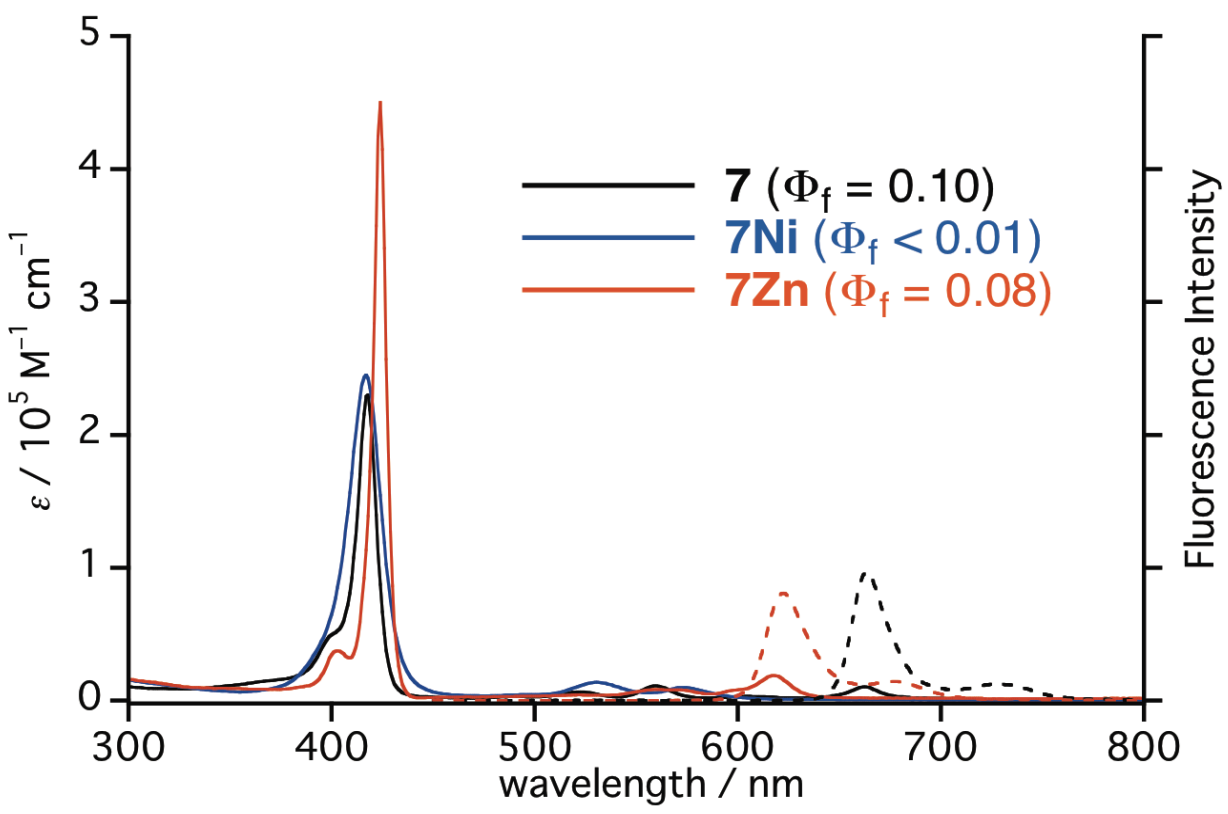

Figure S4-1. UV/Vis absorption (solid) and fluorescence (dashed) spectra of $7 \mathbf{H}_{2}, 7 \mathbf{N i}$, and $7 \mathbf{Z n}$ in THF.

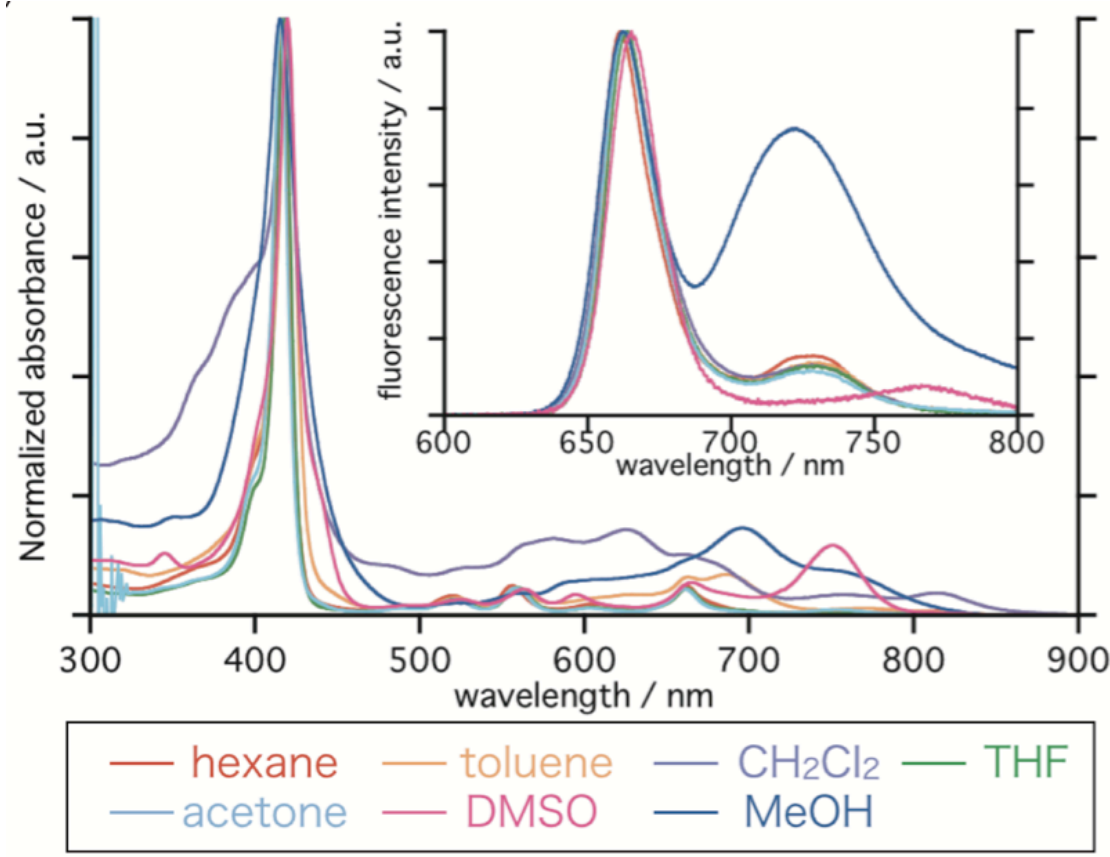

Figure S4-2. UV/Vis absorption and fluorescence spectra of $\mathbf{7} \mathbf{H}_{\mathbf{2}}$ in various solvents. 


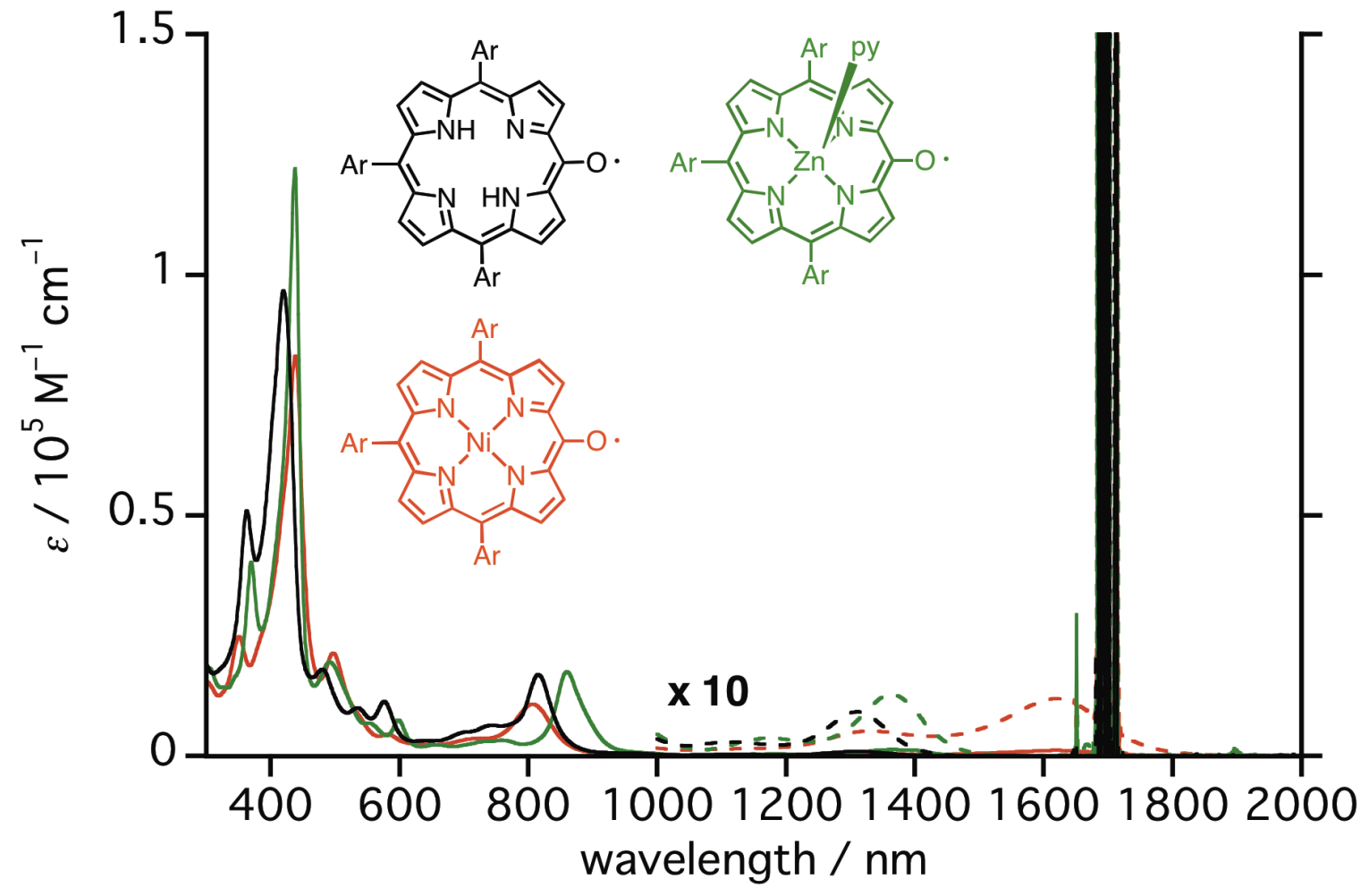

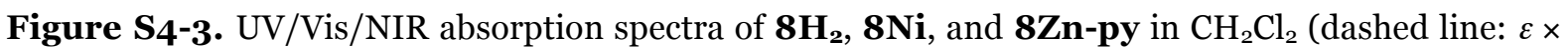
10) 

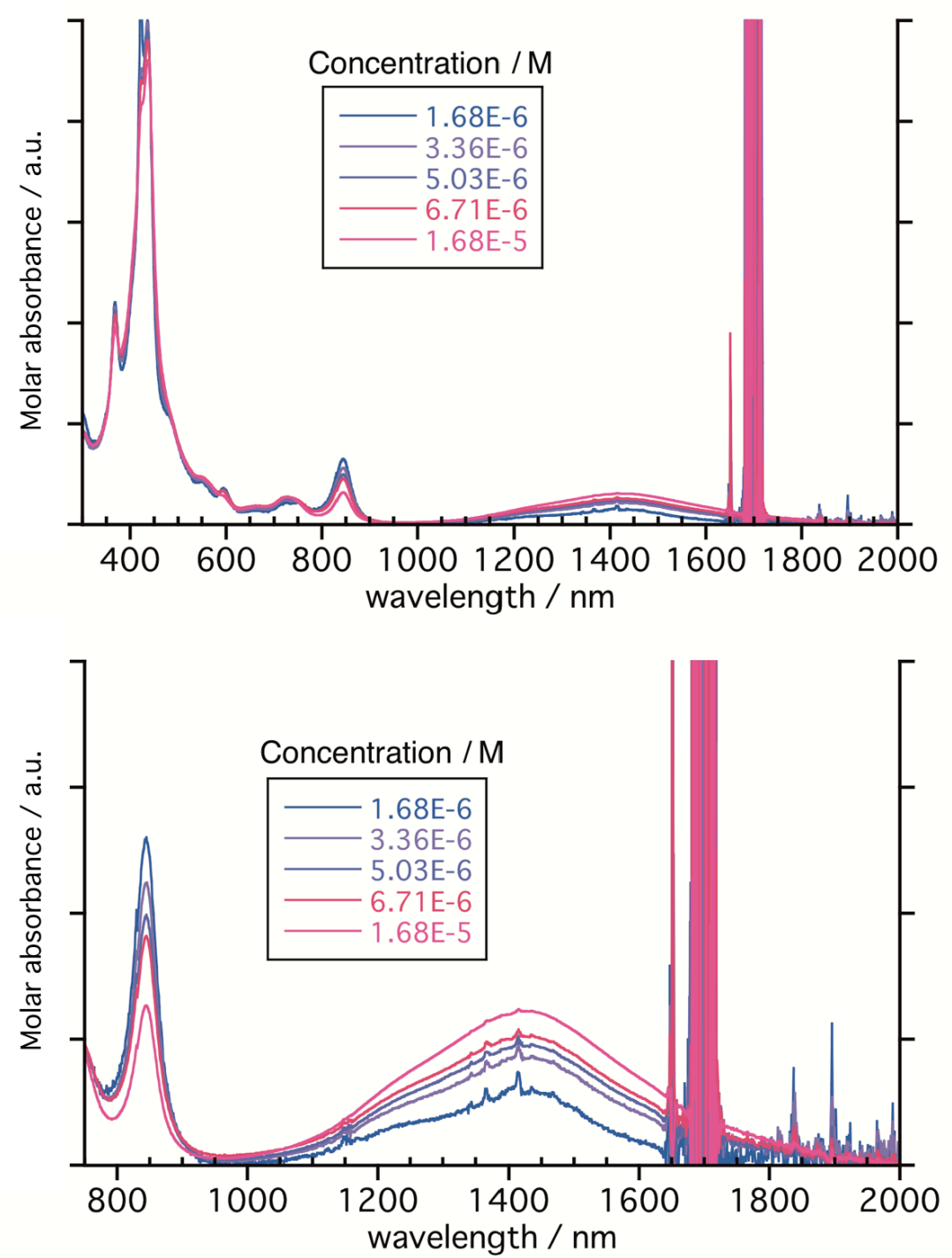

Figure S4-4. UV/Vis absorption spectra of $8 \mathrm{Zn}$ at various concentrations in $\mathrm{CH}_{2} \mathrm{Cl}_{2}$.
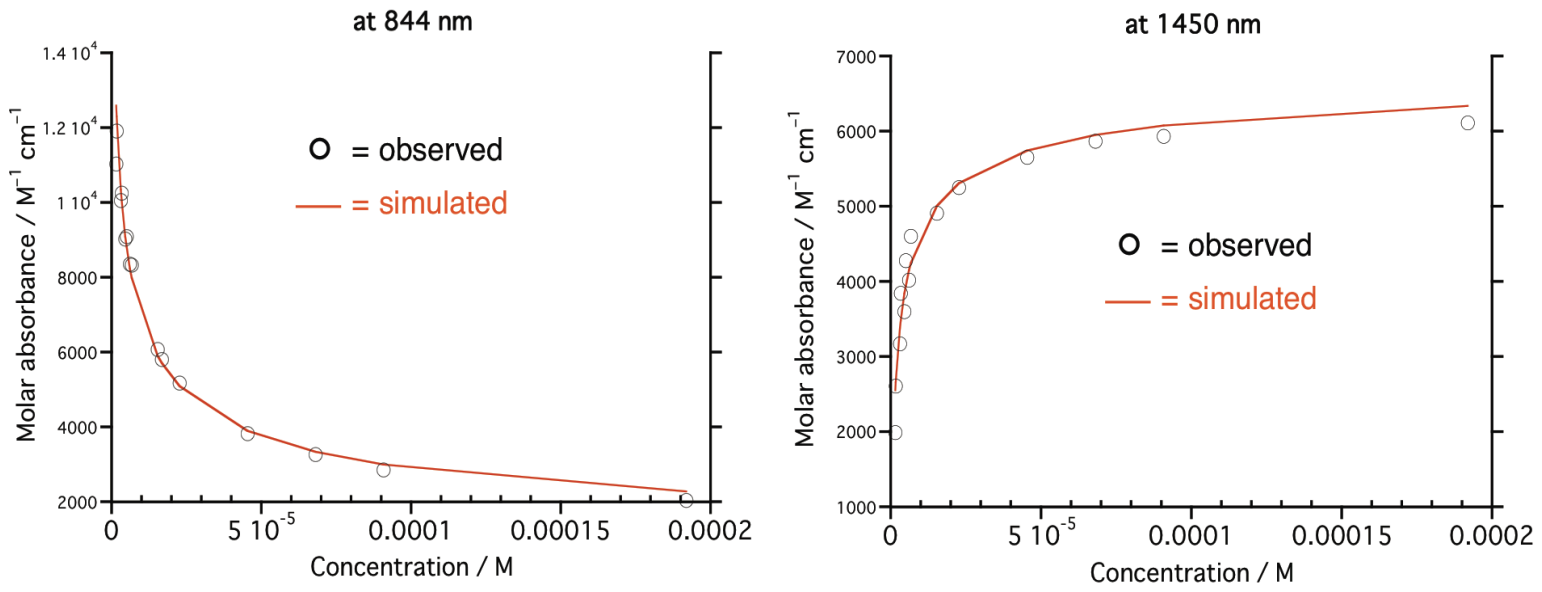

Figure S4-5. Observed and simulated molar absorption of $\mathbf{9 Z n}$ at 844 and $1450 \mathrm{~nm}$ in $\mathrm{CH}_{2} \mathrm{Cl}_{2}$. Simulation was conducted with fitted values; association constant $K_{\mathrm{D}}=3.0 \times 10^{5} \mathrm{M}^{-1}$, molar absorption coefficient $5.8 \times 10^{2} \mathrm{M}^{-1} \mathrm{~cm}^{-1}(844 \mathrm{~nm})$ and $7.0 \times 10^{3} \mathrm{M}^{-1} \mathrm{~cm}^{-1}(1450 \mathrm{~nm})$ for the dimer; molar absorption coefficient $2.0 \times 10^{4} \mathrm{M}^{-1} \mathrm{~cm}^{-1}(844 \mathrm{~nm})$ and $\mathrm{o} \mathrm{M}^{-1} \mathrm{~cm}^{-1}(1450 \mathrm{~nm})$ for the monomer. 


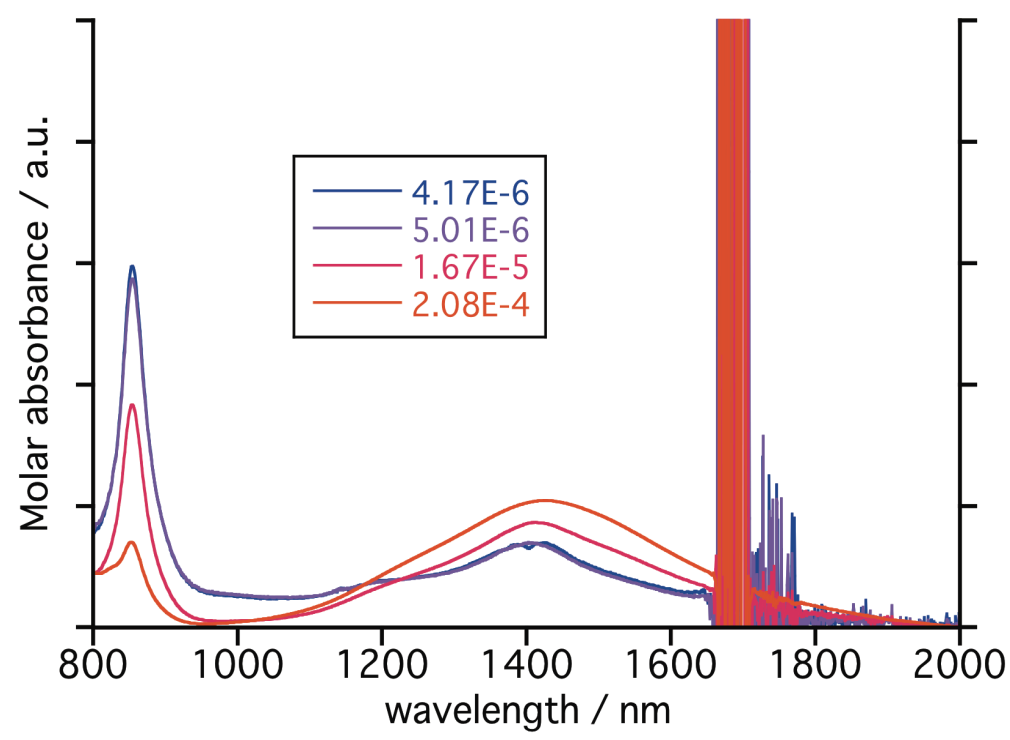

Figure S4-6. UV/Vis absorption spectra of $8 \mathrm{Zn}$ at various concentrations in toluene.
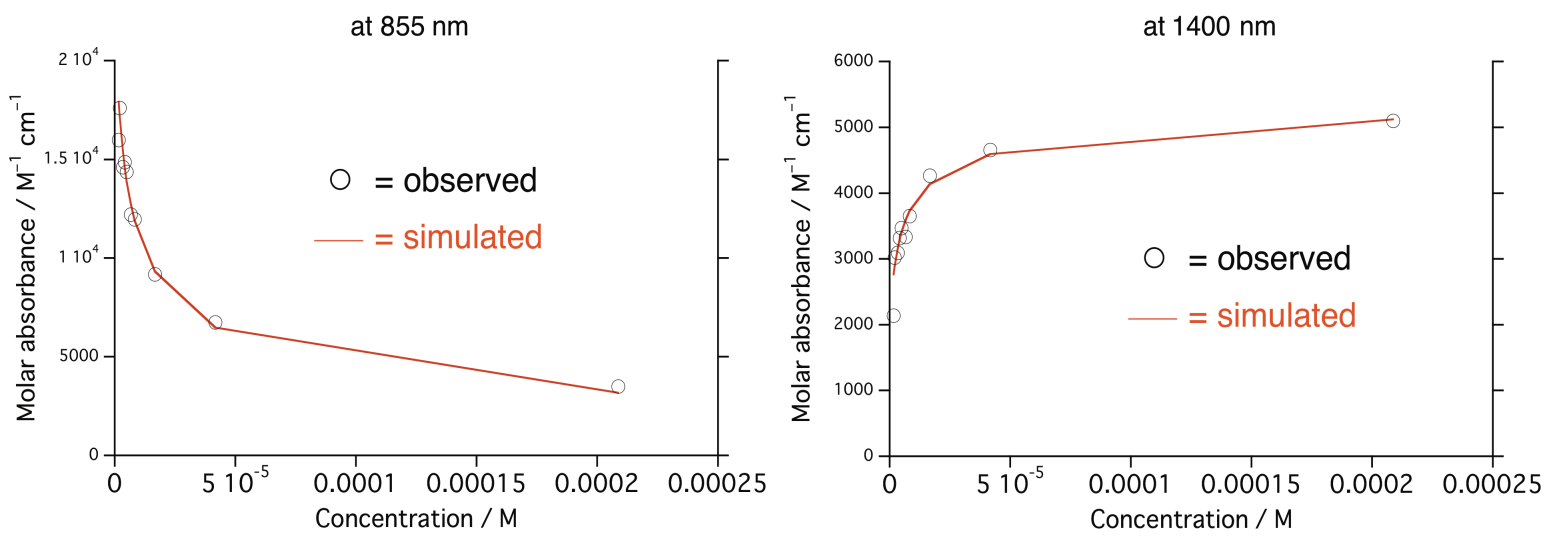

Figure S4-7. Observed and simulated molar absorption of $\mathbf{8 Z n}$ at 855 and $1400 \mathrm{~nm}$ in toluene. Simulation was conducted with fitted values; association constant $K_{\mathrm{D}}=1.1 \times 10^{5} \mathrm{M}^{-1}$, molar absorption coefficient $\mathrm{O} \mathrm{M}^{-1} \mathrm{~cm}^{-1}(855 \mathrm{~nm})$ and $5.6 \times 10^{3} \mathrm{M}^{-1} \mathrm{~cm}^{-1}(1400 \mathrm{~nm})$ for the dimer (9Zn); molar absorption coefficient $2.3 \times 10^{4} \mathrm{M}^{-1} \mathrm{~cm}^{-1}(855 \mathrm{~nm})$ and $2.0 \times 10^{3} \mathrm{M}^{-1} \mathrm{~cm}^{-1}(1400 \mathrm{~nm})$ for the monomer $(\mathbf{8 Z n})$. 


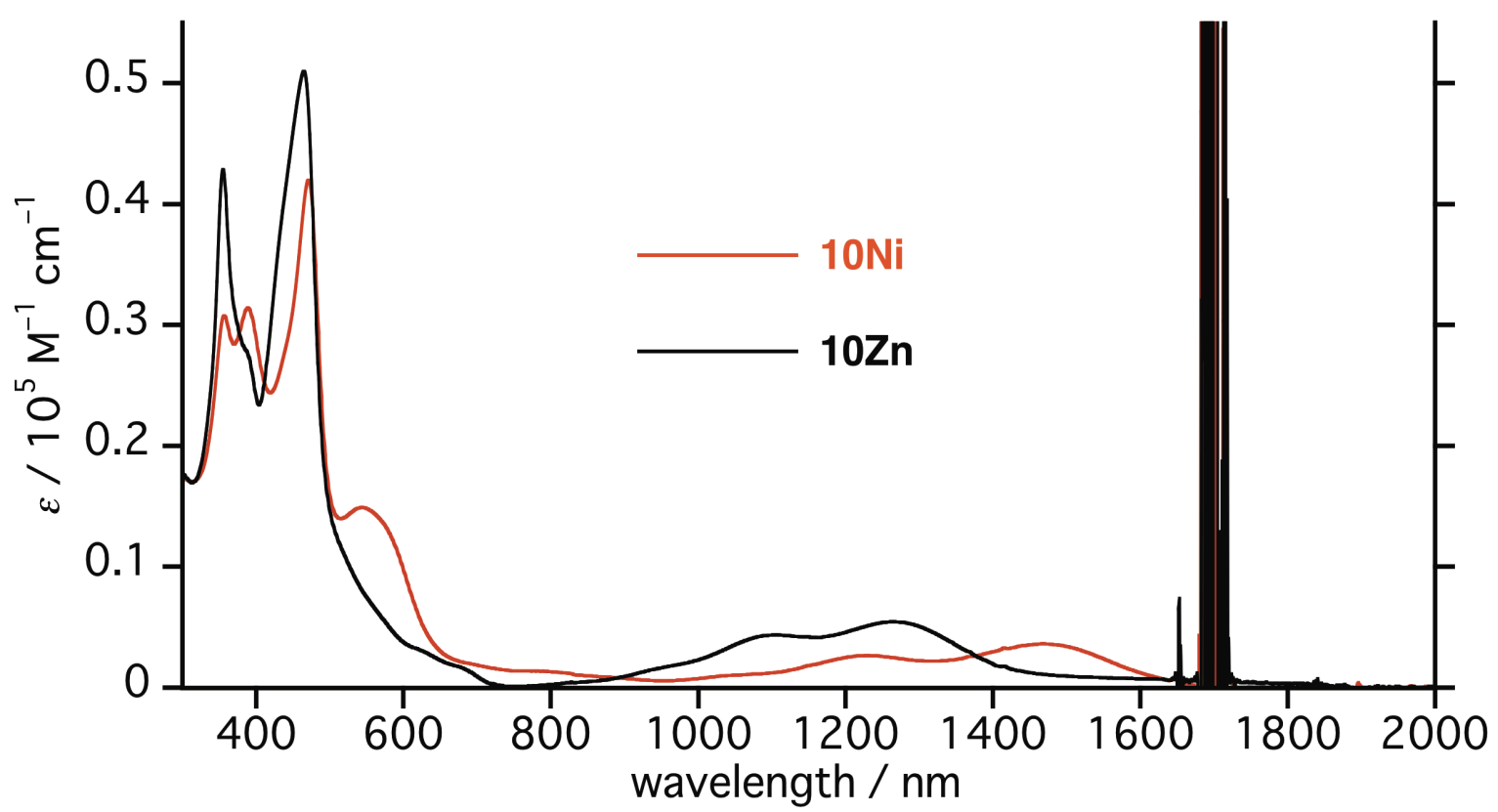

Figure S4-8. UV/Vis/NIR absorption spectra of $10 \mathrm{Ni}$ and $10 \mathrm{Zn}$ in $\mathrm{CH}_{2} \mathrm{Cl}_{2}$. 


\section{X-Ray Crystallographic Analysis}

X-Ray crystallographic data were recorded by using a Rigaku XtaLAB P-20o system. The structures were solved by using direct methods (SIR-97 ${ }^{\mathrm{S} 1}$ or SHELX-97 $7^{\mathrm{S} 2}$ ). Structure refinements were carried out by using SHELXL-98. CCDC numbers 1408586 (7Ni), $1408590\left(\mathbf{8 H} \mathbf{H}_{\mathbf{2}}\right), 1408587(\mathbf{8 N i})$, 1408588 (8Zn-py), 1423041 (9Zn), and 1408589 (10Ni) contain supplementary crystallographic data for this paper. These data can be obtained free of charge from the Cambridge Crystallographic Data Centre via www.ccdc.cam.ac.uk/data_request/cif.

Table S5-1. Crystal data of $7 \mathbf{N i}, 8 \mathrm{H}_{2}, 8 \mathrm{Ni}$, and $8 \mathrm{Zn}-\mathbf{p y}$.

\begin{tabular}{|c|c|c|c|c|}
\hline Compound & $7 \mathrm{Ni}$ & $\mathbf{8} \mathbf{H}_{2}$ & $8 \mathbf{N i}$ & 8Zn-py \\
\hline sum formula & $\mathrm{C}_{63} \mathrm{H}_{76} \mathrm{~N}_{4} \mathrm{NiO}_{2}$ & $\mathrm{C}_{63} \mathrm{H}_{75} \mathrm{Cl}_{2} \mathrm{~N}_{4} \mathrm{O}$ & $\mathrm{C}_{62} \mathrm{H}_{71} \mathrm{~N}_{4} \mathrm{NiO}$ & $\mathrm{C}_{69} \mathrm{H}_{77} \mathrm{~N}_{5} \mathrm{O}_{1.5} \mathrm{Zn}$ \\
\hline formula weight $/ \mathrm{mol}^{-1}$ & 979.97 & 975.14 & 946.92 & 1065.75 \\
\hline crystal dimensions / $\mathbf{m m} 3$ & $0.05 \times 0.03 \times 0.01$ & $0.20 \times 0.08 \times 0.04$ & $0.10 \times 0.08 \times 0.04$ & $0.07 \times 0.07 \times 0.01$ \\
\hline crystal description & orange & brawn & brown & brown \\
\hline crystal system & triclinic & triclinic & triclinic & triclinic \\
\hline space group & $P-1$ & $P-1$ & $P-1$ & $P-1$ \\
\hline$a / \AA$ & $9.011(6)$ & $11.665(2)$ & $11.8998(18)$ & $13.2483(7)$ \\
\hline b / $\mathbf{A}$ & $15.824(8)$ & $15.633(3)$ & $16.0028(5)$ & $14.6134(10)$ \\
\hline c/ / & $20.375(8)$ & $15.927(4)$ & $16.0810(6)$ & $18.0800(18)$ \\
\hline$\alpha /^{\circ}$ & 70.31(4) & $104.962(7)$ & 64.42(3) & $93.09(3)$ \\
\hline$\beta /{ }^{\circ}$ & $86.42(5)$ & $91.926(9)$ & $80.99(3)$ & $104.28(4)$ \\
\hline$\gamma /{ }^{\circ}$ & $87.73(5)$ & 95.774(5) & $70.34(2)$ & $116.22(2)$ \\
\hline$V / \AA^{3}$ & $2730(3)$ & 2786.4(10) & $2600.9(8)$ & 2989.4(4) \\
\hline$Z$ & 2 & 2 & 2 & 2 \\
\hline$\rho_{\text {calcd. }} / \mathrm{g} \mathrm{cm}^{-3}$ & 1.192 & 1.162 & 1.209 & 1.184 \\
\hline$\mu / \mathbf{m m}^{-1}$ & 0.863 & 1.377 & 0.875 & 0.928 \\
\hline$F(\mathbf{0 0 0})$ & 1052 & 1046 & 1014 & 1136 \\
\hline $\mathbf{T} / \mathbf{K}$ & $93(2)$ & $93(2)$ & $93(2)$ & $93(2)$ \\
\hline$\theta$ range & $6.51-62.50$ & $6.66-67.50$ & $6.80-62.99$ & $6.58-62.50$ \\
\hline index ranges & $-10<h<10$ & $-13<\mathrm{h}<13$ & $-13<h<13$ & $-14<h<15$ \\
\hline & $-15<k<18$ & $-18<\mathrm{k}<18$ & $-18<k<17$ & $-16<k<16$ \\
\hline & $-23<l<23$ & $-19<1<16$ & $-18<l<17$ & $-20<l<19$ \\
\hline reflections measured & 32121 & 35656 & 31392 & 35041 \\
\hline reflections independent & 8507 & 9729 & 8221 & 9284 \\
\hline reflections unique & 4523 & 7752 & 7039 & 6932 \\
\hline $\boldsymbol{R}_{\text {int }}$ & 0.1463 & 0.0484 & 0.0282 & 0.0580 \\
\hline$R_{1}[I>2 \sigma(I)]$ & 0.0984 & 0.0906 & 0.0409 & 0.0842 \\
\hline$R_{\mathrm{w}}$ (all data) & 0.3013 & 0.2763 & 0.1171 & 0.2458 \\
\hline data/restrains/parameters & $8507 / 0 / 652$ & $9729 / 20 / 650$ & $8221 / 18 / 658$ & $9284 / 63 / 730$ \\
\hline GOF & 1.040 & 1.010 & 1.013 & 1.032 \\
\hline residual electron density & $-0.503 / 1.600$ & $-1.469 / 1.547$ & $-0.373 / 0.593$ & $-0.754 / 1.706$ \\
\hline
\end{tabular}


Table S5-2. Crystal data of $9 \mathrm{Zn}$, and $10 \mathrm{Ni}$.

\begin{tabular}{|c|c|c|}
\hline Compound & 9Zn & 10Ni \\
\hline sum formula & $\mathrm{C}_{66} \mathrm{H}_{79} \mathrm{~N}_{4} \mathrm{O}_{2} \mathrm{Zn}$ & $\mathrm{C}_{62.2} \mathrm{H}_{71.4} \mathrm{Cl}_{16.4} \mathrm{~N}_{4} \mathrm{NiOSb}$ \\
\hline formula weight $/ \mathbf{m o l}^{-1}$ & $1025 \cdot 72$ & 1298.36 \\
\hline crystal dimensions / $/ \mathbf{m m}^{3}$ & $0.30 \times 0.04 \times 0.02$ & $0.13 \times 0.03 \times 0.01$ \\
\hline crystal description & brown & brown \\
\hline crystal system & triclinic & triclinic \\
\hline space group & $P-1$ & $P-1$ \\
\hline$a / \AA$ & $12.5135(16)$ & $10.130(2)$ \\
\hline b / $\AA \AA$ & $13.6400(11)$ & $16.127(4)$ \\
\hline c/ $/ \AA$ & $18.3923(15)$ & $20.150(8)$ \\
\hline$\alpha /^{\circ}$ & 110.787(9) & 92.98(4) \\
\hline$\beta /{ }^{\circ}$ & 99.471(14) & $97.03(6)$ \\
\hline$\gamma /{ }^{\circ}$ & $95.73(2)$ & $105.76(3)$ \\
\hline$V / \AA^{3}$ & $2852.0(6)$ & $3131.7(16)$ \\
\hline$Z$ & 2 & 2 \\
\hline$\rho_{\text {calcd. }} / \mathrm{g} \mathrm{cm}^{-3}$ & 1.194 & 1.377 \\
\hline$\mu / \mathbf{m m}^{-1}$ & 0.952 & 6.631 \\
\hline$F(000)$ & 1098 & 1336.8 \\
\hline $\mathbf{T} / \mathbf{K}$ & $93(2)$ & $93(2)$ \\
\hline$\theta$ range & $6.52-63.00$ & $6.59-67.50$ \\
\hline \multirow[t]{3}{*}{ index ranges } & $-13<h<14$ & $-12<\mathrm{h}<12$ \\
\hline & $-15<k<15$ & $-19<\mathrm{k}<16$ \\
\hline & $-21<l<21$ & $-24<1<24$ \\
\hline reflections measured & 34523 & 40046 \\
\hline reflections independent & 9231 & 10946 \\
\hline reflections unique & 7303 & 6901 \\
\hline $\boldsymbol{R}_{\text {int }}$ & 0.0633 & 0.1336 \\
\hline$R_{1}[I>2 \sigma(I)]$ & 0.0453 & 0.0570 \\
\hline$R_{\mathrm{w}}$ (all data) & 0.1210 & 0.1506 \\
\hline data/restrains/parameters & $9051 / 0 / 721$ & $10946 / 56 / 752$ \\
\hline GOF & 1.026 & 1.003 \\
\hline residual electron density & $-0.509 / 1.061$ & $-1.168 / 1.237$ \\
\hline
\end{tabular}




\section{Electrochemical Analysis}

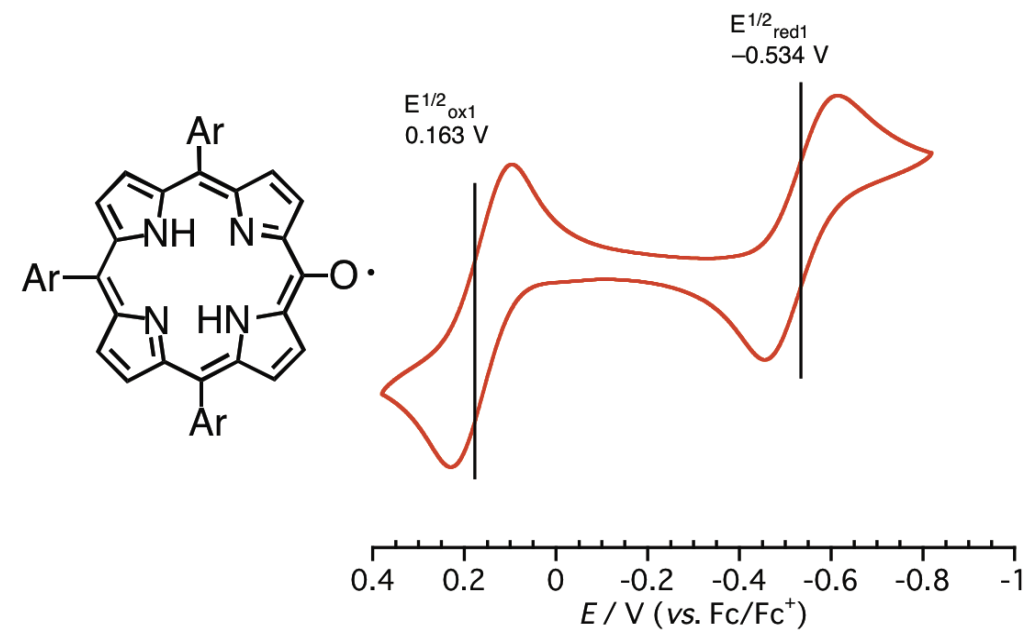

Figure S6-1. Cyclic voltammogram of $\mathbf{8} \mathbf{H}_{2}$ in $\mathrm{CH}_{2} \mathrm{Cl}_{2}$ (scan rate: $0.05 \mathrm{~V} / \mathrm{s}$, supporting electrolyte: 0.1 $\mathrm{M} n \mathrm{Bu}_{4} \mathrm{NPF}_{6}$, working electrode: Pt, counter electrode: Pt wire, reference electrode: $\mathrm{Ag} / \mathrm{o.01} \mathrm{M}$ $\left.\mathrm{AgClO}_{4}\right)$.

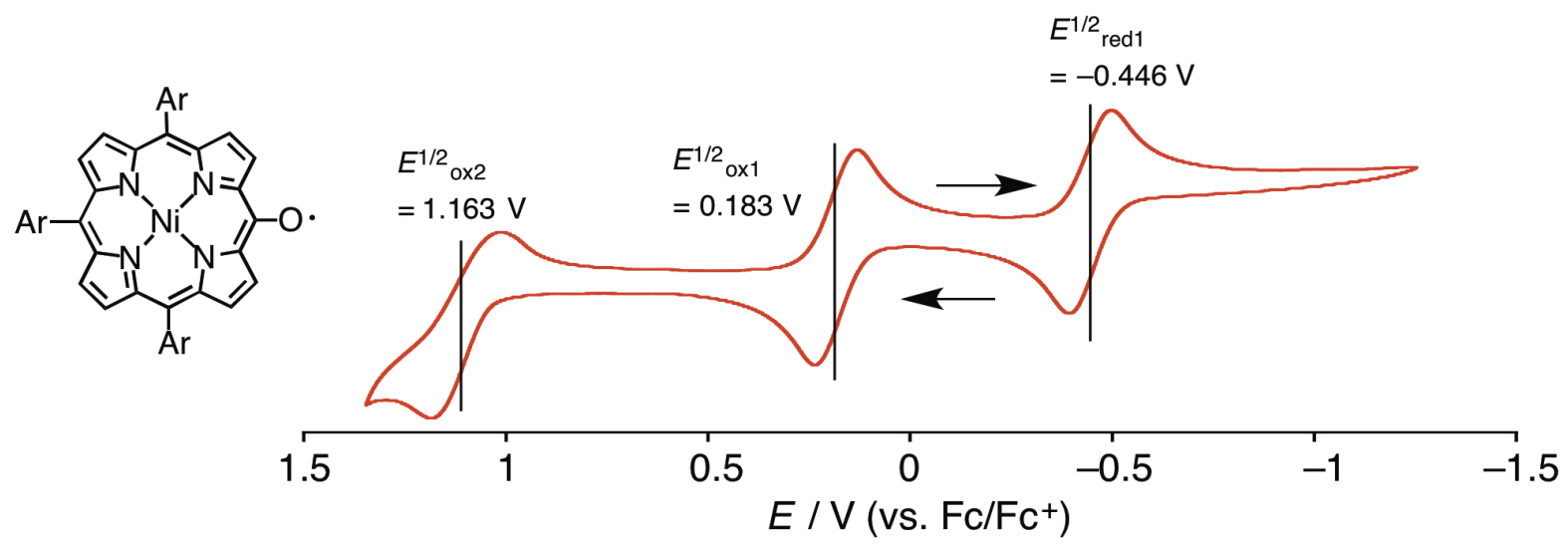

Figure S6-2. Cyclic voltammogram of $\mathbf{8 N i}$ in $\mathrm{CH}_{2} \mathrm{Cl}_{2}$ (scan rate: $0.05 \mathrm{~V} / \mathrm{s}$, supporting electrolyte: 0.1 $\mathrm{M} n \mathrm{Bu}_{4} \mathrm{NPF}_{6}$, working electrode: Pt, counter electrode: Pt wire, reference electrode: Ag/o.01 M $\left.\mathrm{AgClO}_{4}\right)$. 


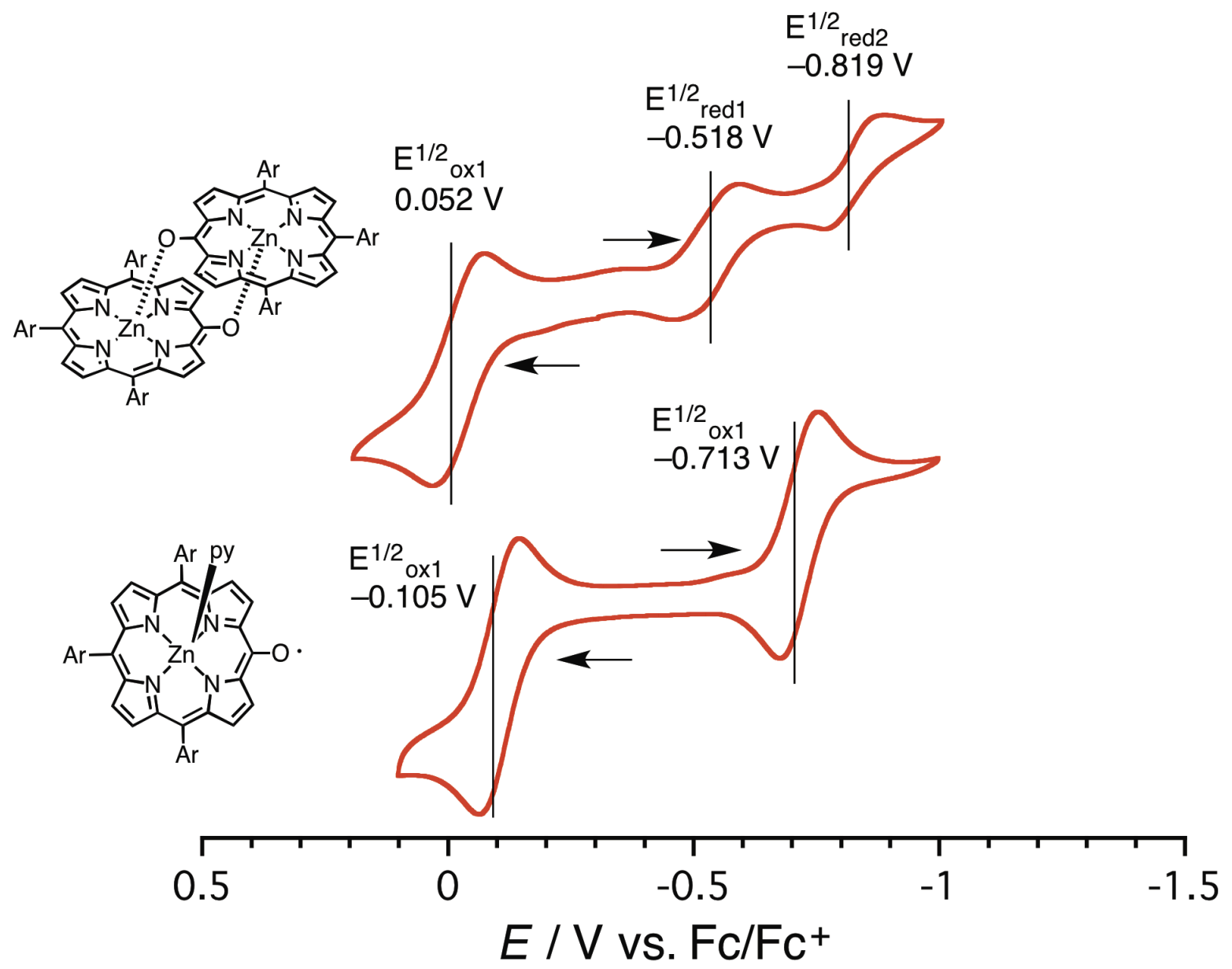

Figure S6-3. Cyclic voltammograms of $\mathbf{9 Z n}$ and 8Zn-py in $\mathrm{CH}_{2} \mathrm{Cl}_{2}$ (scan rate: $0.05 \mathrm{~V} / \mathrm{s}$, supporting electrolyte: $0.1 \mathrm{M} n \mathrm{Bu}_{4} \mathrm{NPF}_{6}$, working electrode: Pt, counter electrode: Pt wire, reference electrode: $\left.\mathrm{Ag} / \mathrm{o.01} \mathrm{M} \mathrm{AgClO}_{4}\right)$. 


\section{Temperature Dependent Magnetic Susceptibility}

Magnetic susceptibility was measured for the powder sample with the temperature range from 2 to $300 \mathrm{~K}$ at $0.5 \mathrm{~T}$ magnetic fields by a Quantum Design MPMS-2S instrument. Observed $\chi \mathrm{T}$ values were reproduced by using Bleaney-Bowers singlet-triplet model and Curie-Weiss model.

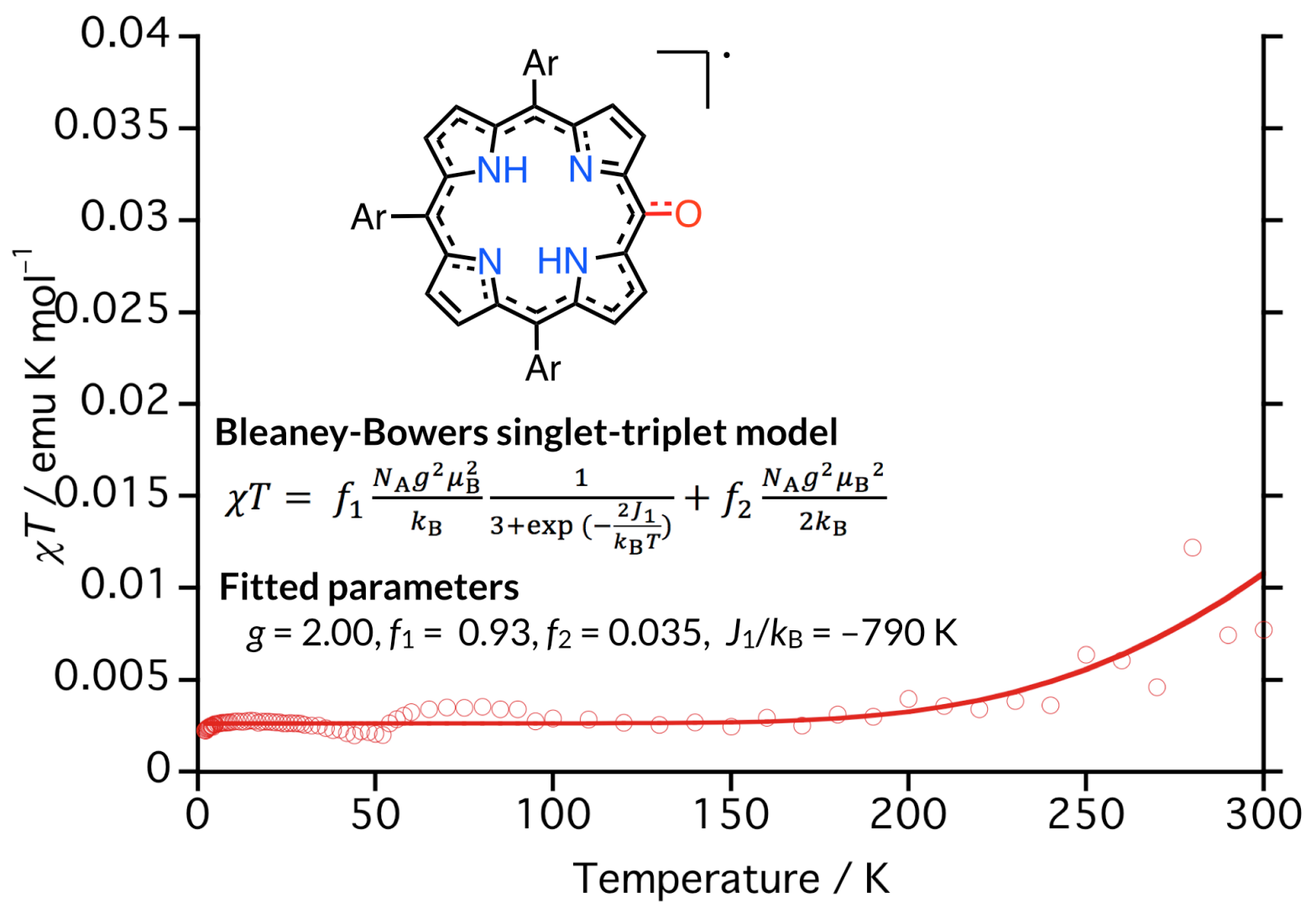

Figure S7-1. Observed (circles) and simulated (solid line) $\chi \mathrm{T}$ values of $\mathbf{8} \mathbf{H}_{\mathbf{2}}$. 


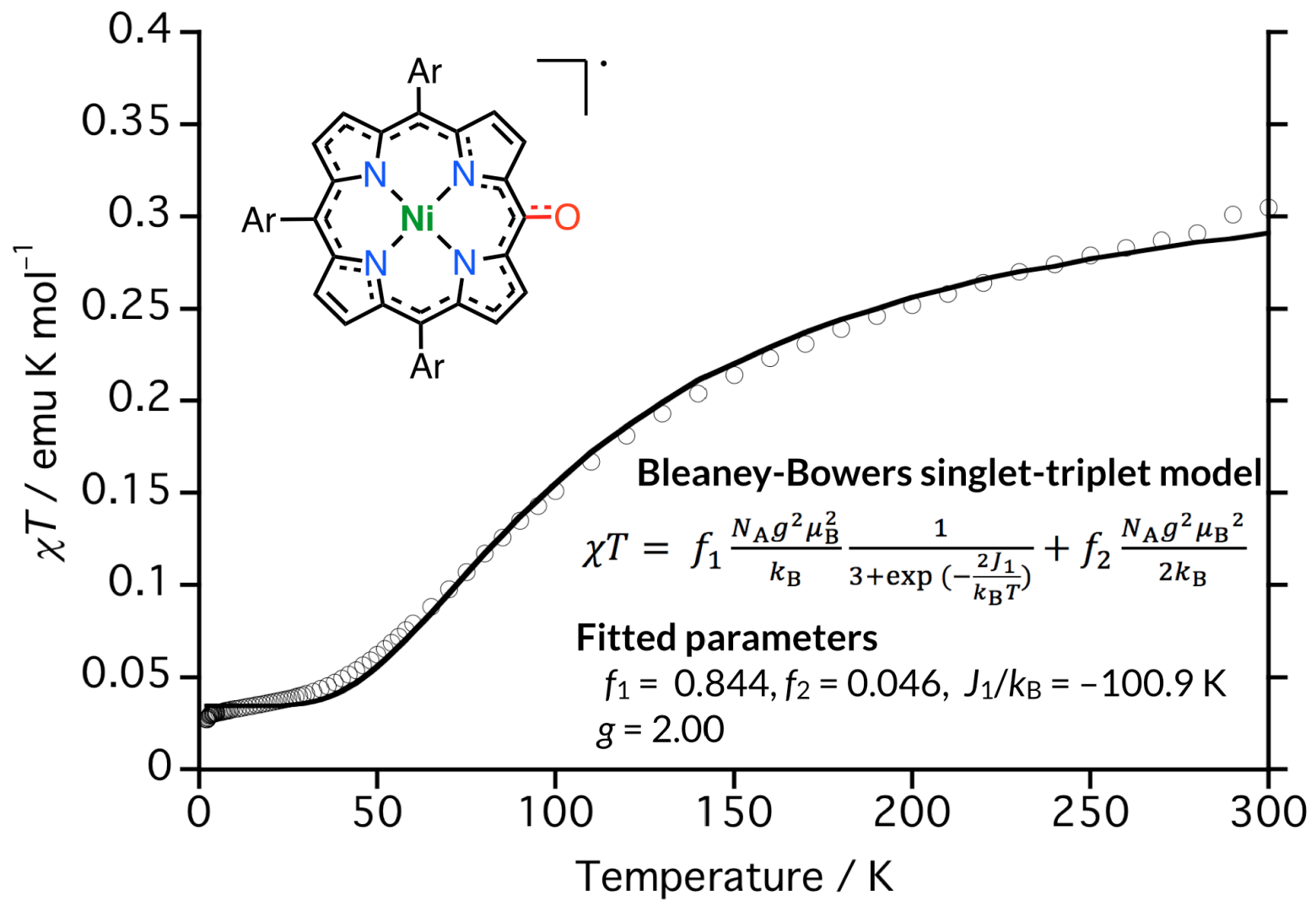

Figure S7-2. Observed (circles) and simulated (solid line) $\chi \mathrm{T}$ values of $\mathbf{8 N i}$.

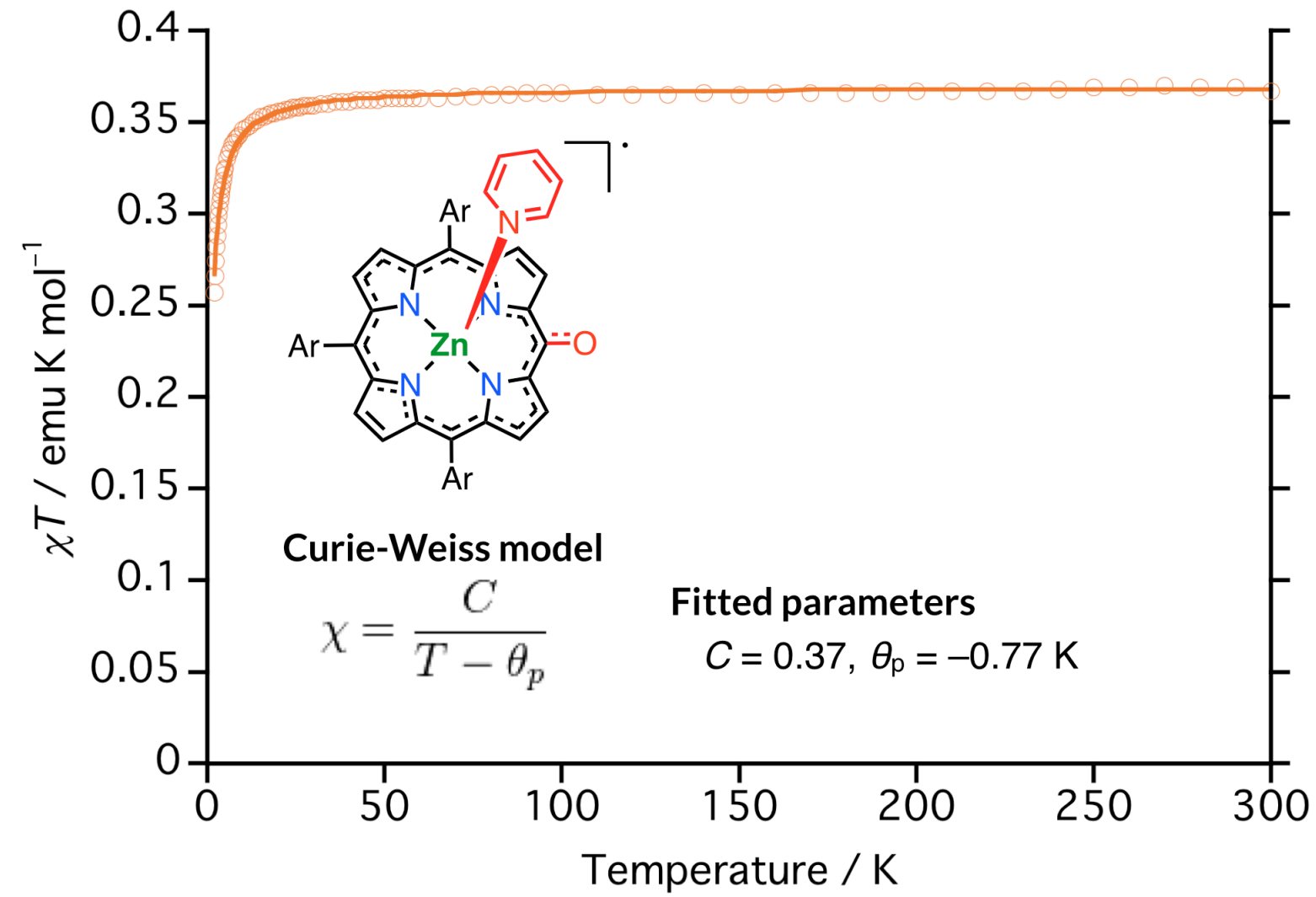

Figure S7-3. Observed (circles) and simulated (solid line) $\chi$ T values of 8Zn-py. 


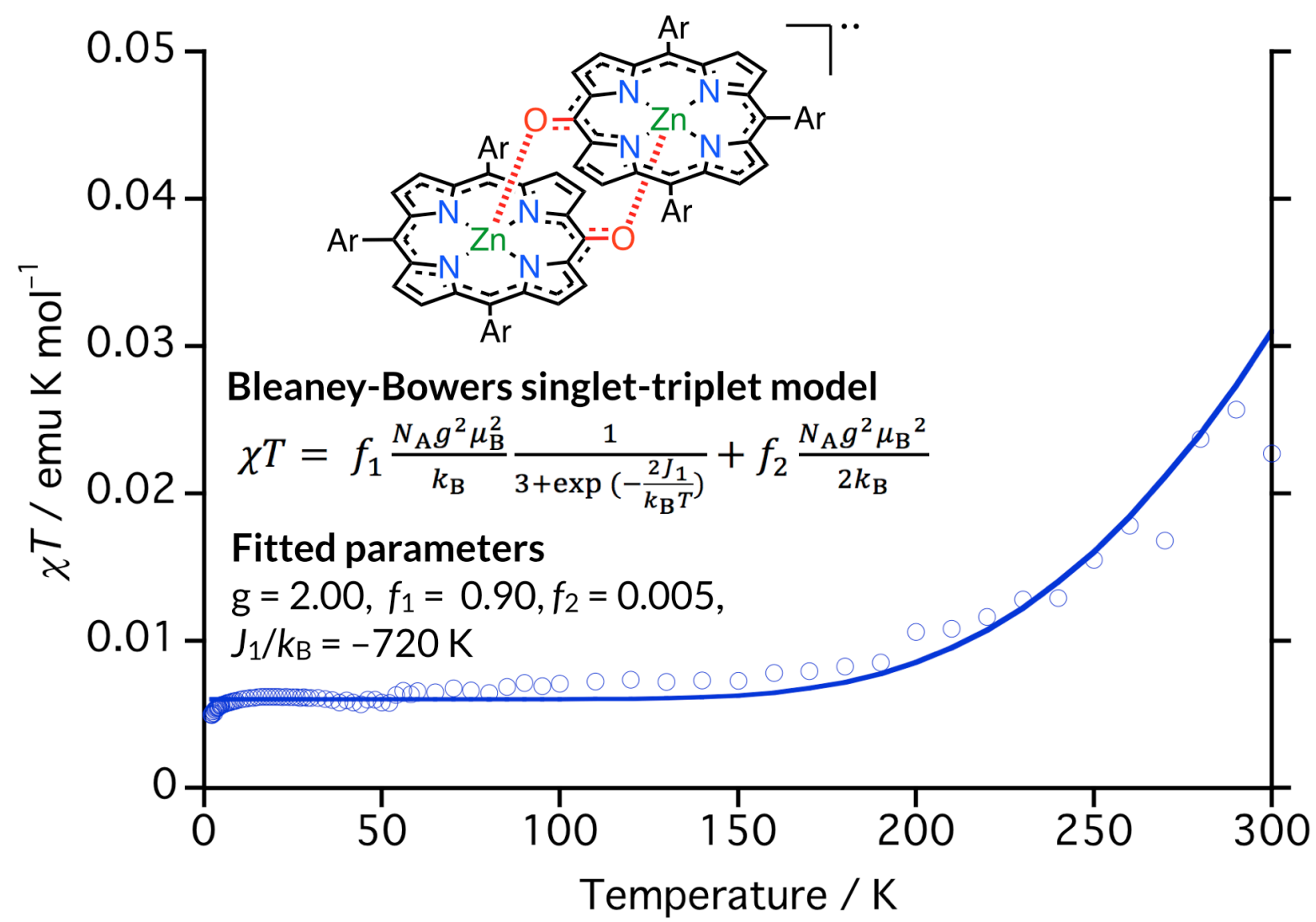

Figure S7-4. Observed (circles) and simulated (solid line) $\chi \mathrm{T}$ values of $\mathbf{9 Z \mathbf { Z n }}$. 


\section{Theoretical Calculations}

For DFT calculations, molecular geometries were optimized on the basis of X-ray crystal structures except for $10 Z \mathbf{Z}$. The structure of $\mathbf{1 0 Z n}$ was optimized on the basis of X-ray crystal structure of 10Ni. All tert-butyl groups were displaced with hydrogen atoms for simplicity. The optimized molecular geometries are shown in section 11.

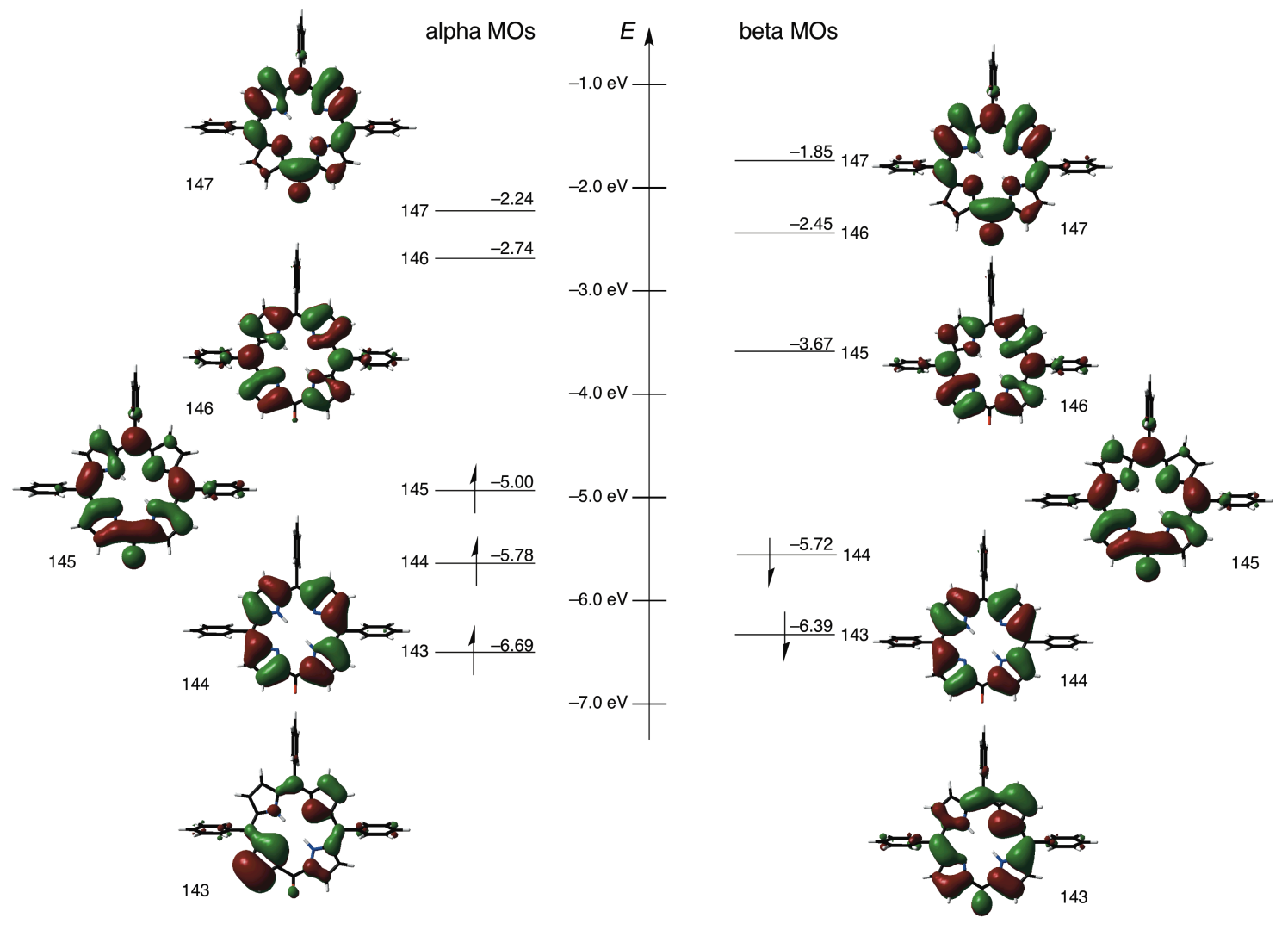

Figure S8-1. Selected molecular orbitals of $\mathbf{8} \mathbf{H}_{2}$ (top view) calculated at UB3LYP/6-311G(d) level.

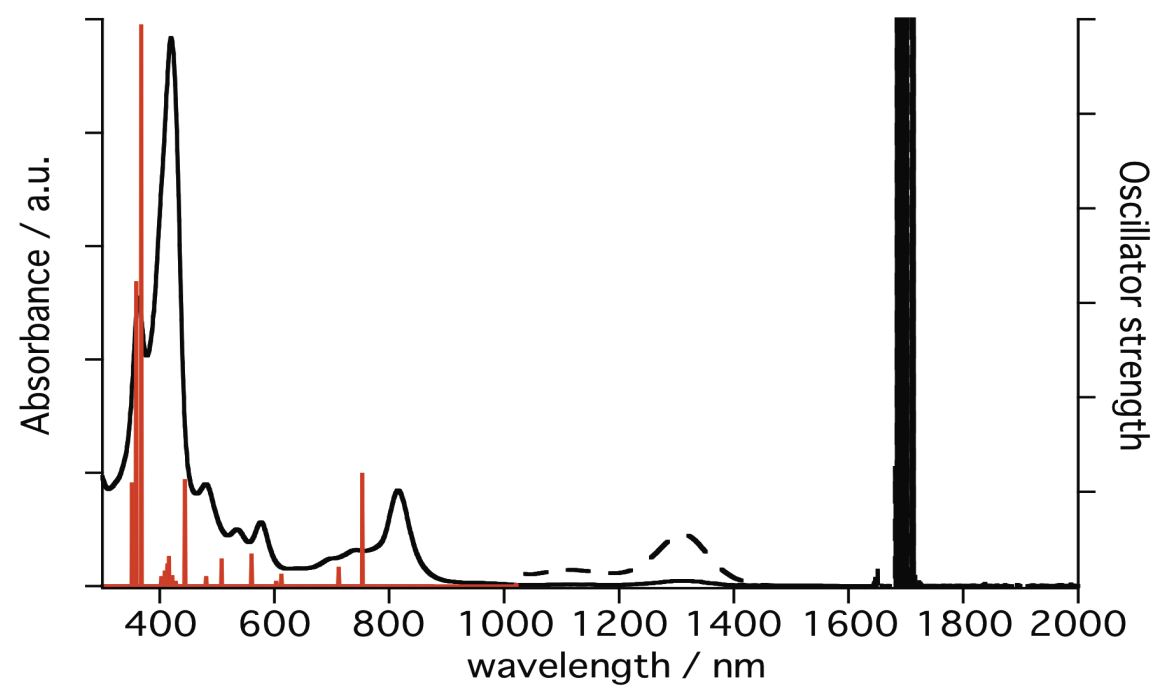

Figure S8-2. UV/Vis/NIR absorption spectrum (black) and oscillator strengths (red) of $\mathbf{8 H}_{\mathbf{2}}$

calculated at UB3LYP/6-311G(d) level. Selected oscillator strengths: $f=0.0015(1021 \mathrm{~nm}), 0.1182(753$ $\mathrm{nm}), 0.1113(444 \mathrm{~nm}), 0.5927(368 \mathrm{~nm}), 0.3208(359 \mathrm{~nm})$. 

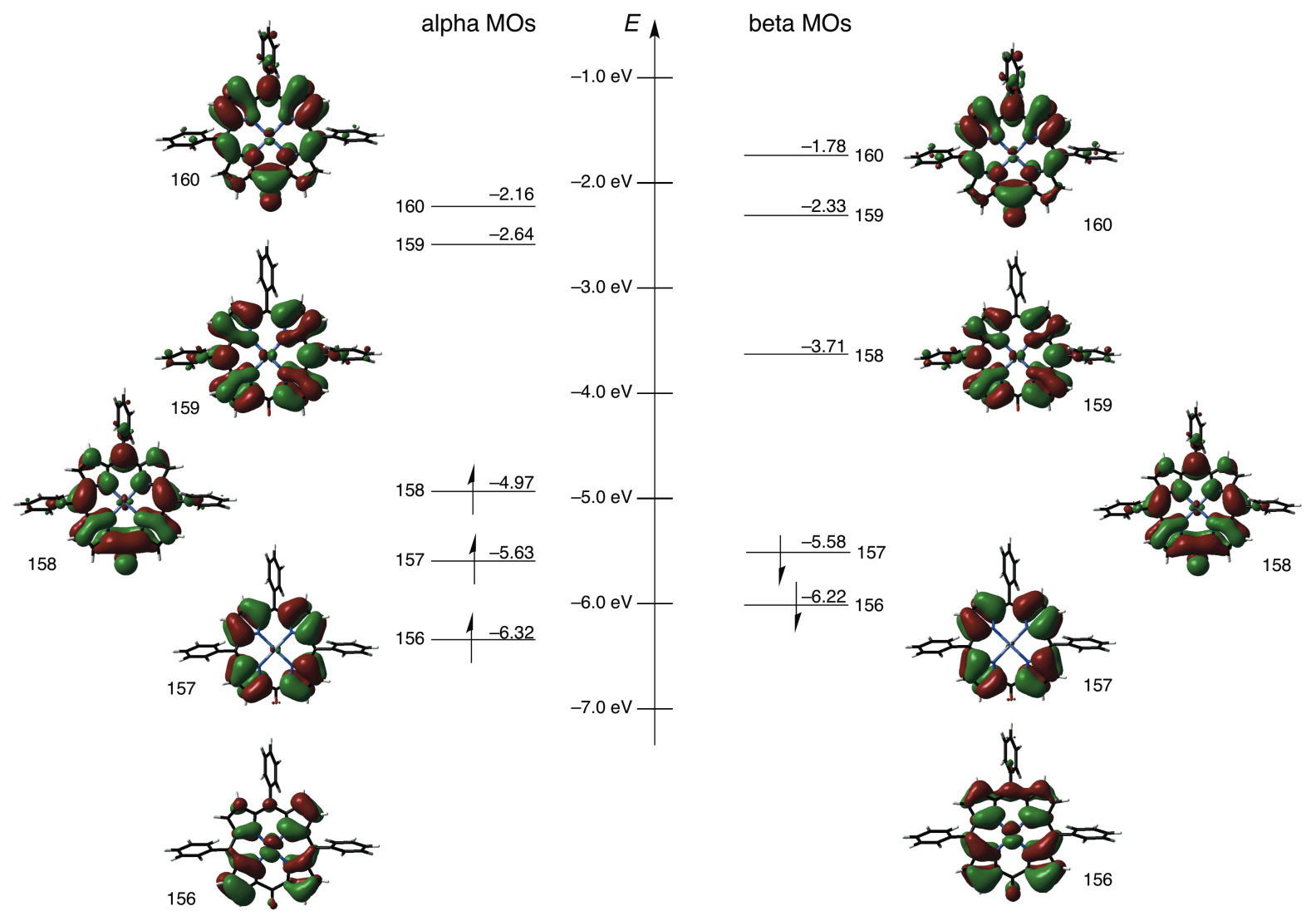

Figure S8-3. Selected molecular orbitals of 8Ni (top view) calculated at UB3LYP/6-311G*(C,H,N,O)-cc-pVQZ(Ni) level..

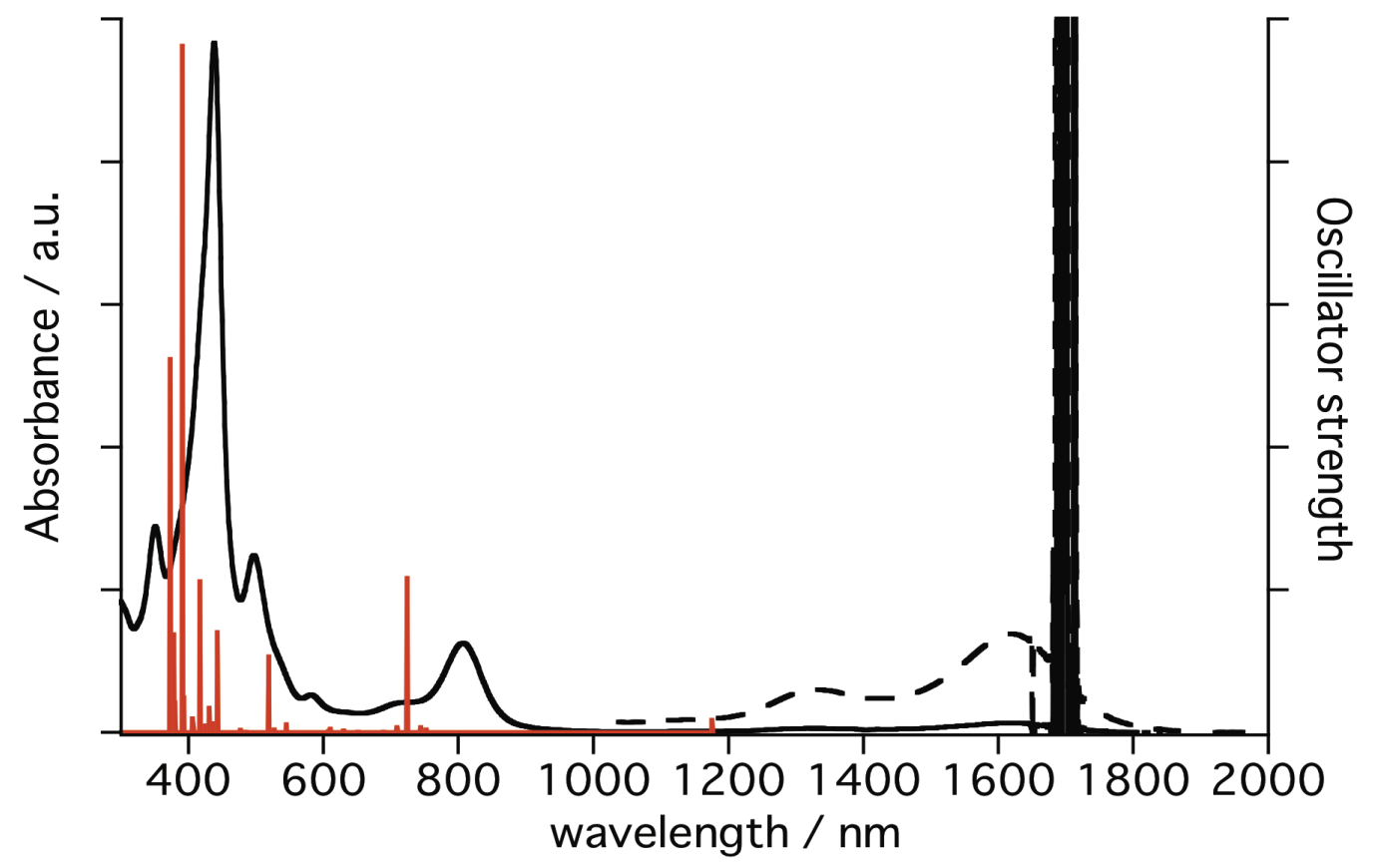

Figure S8-4. UV/Vis/NIR absorption spectrum (black) and oscillator strengths (red) of $\mathbf{8 N i}$ calculated at UB3LYP/6-311G(d) (C,H,N,O)-cc-pVQZ(Ni) level. Selected oscillator strengths: $f=$ $0.0074(1176 \mathrm{~nm}), 0.0952(724 \mathrm{~nm}), 0.0467(519 \mathrm{~nm}), 0,4233(391 \mathrm{~nm}), 0.2300(373 \mathrm{~nm})$. 


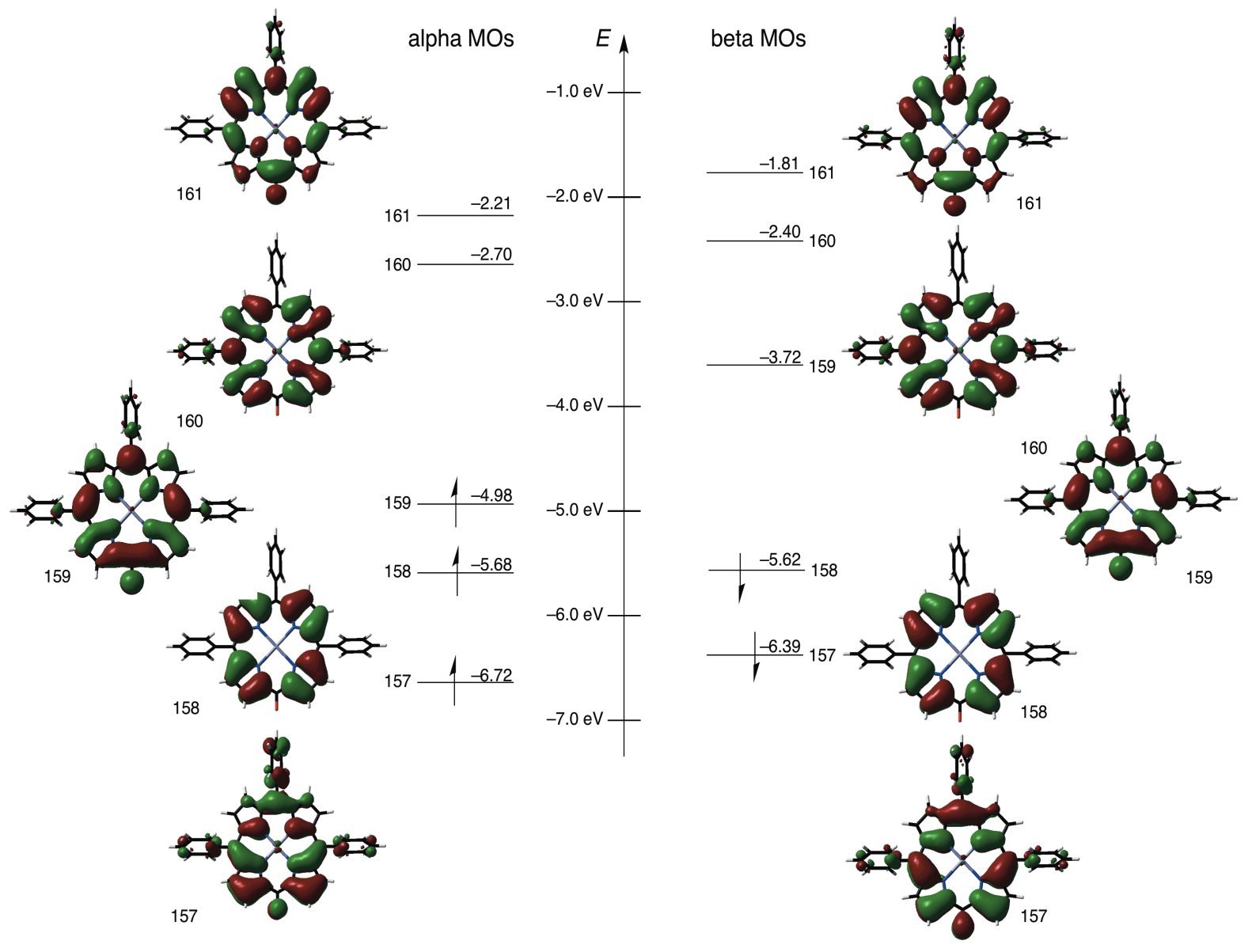

Figure S8-5. Selected molecular orbitals of $\mathbf{8 Z n}$ (top view) calculated at UB3LYP/6-311G*(C,H,N,O)-cc-pVQZ(Zn) level..

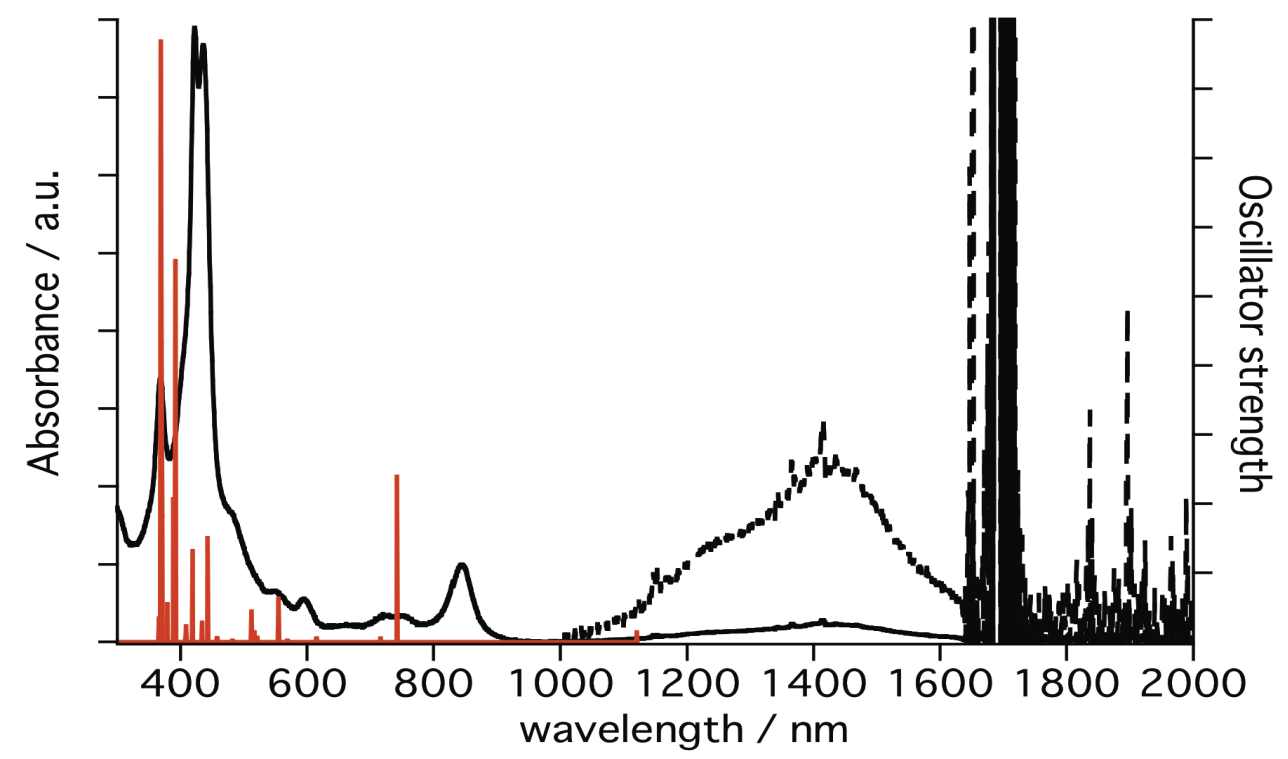

Figure S8-6. UV/Vis/NIR absorption spectrum (at $1.68 \mu \mathrm{M}$, black) and oscillator strengths (red) of 8Zn calculated at UB3LYP/6-311G(d) $(\mathrm{C}, \mathrm{H}, \mathrm{N}, \mathrm{O})-\mathrm{cc}-\mathrm{pVQZ}(\mathrm{Ni})$ level. Selected oscillator strengths: $f=$ 0.0071 (1121 nm), 0.1196 (742 nm), 0.0314 (555 nm), 0.2754 (392 nm), 0.4342 (369 nm). 


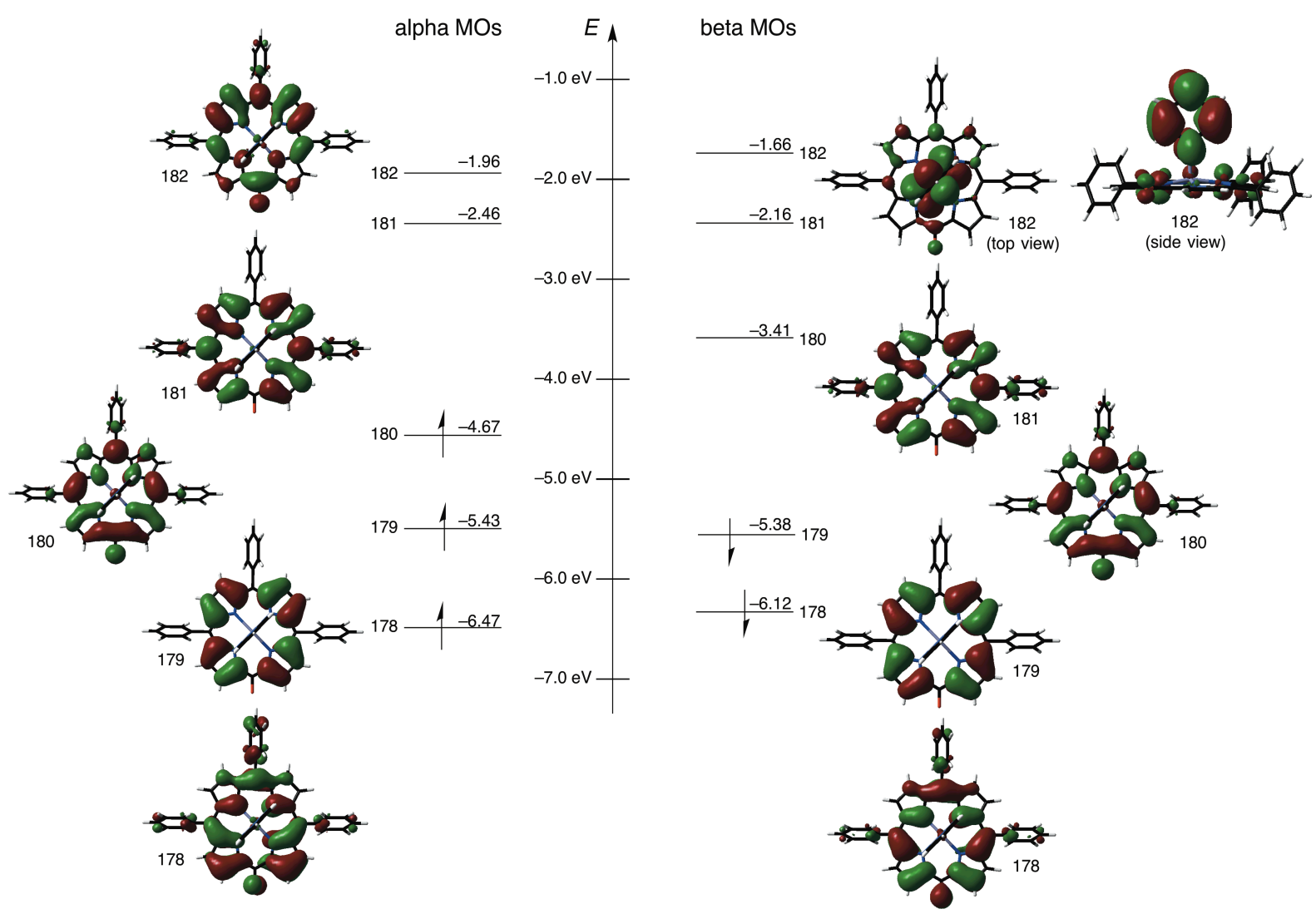

Figure S8-7. Selected molecular orbitals of 8Zn-py (top view) calculated at UB3LYP/6-311G(d) (C,H,N,O)-cc-pVQZ (Zn) level.

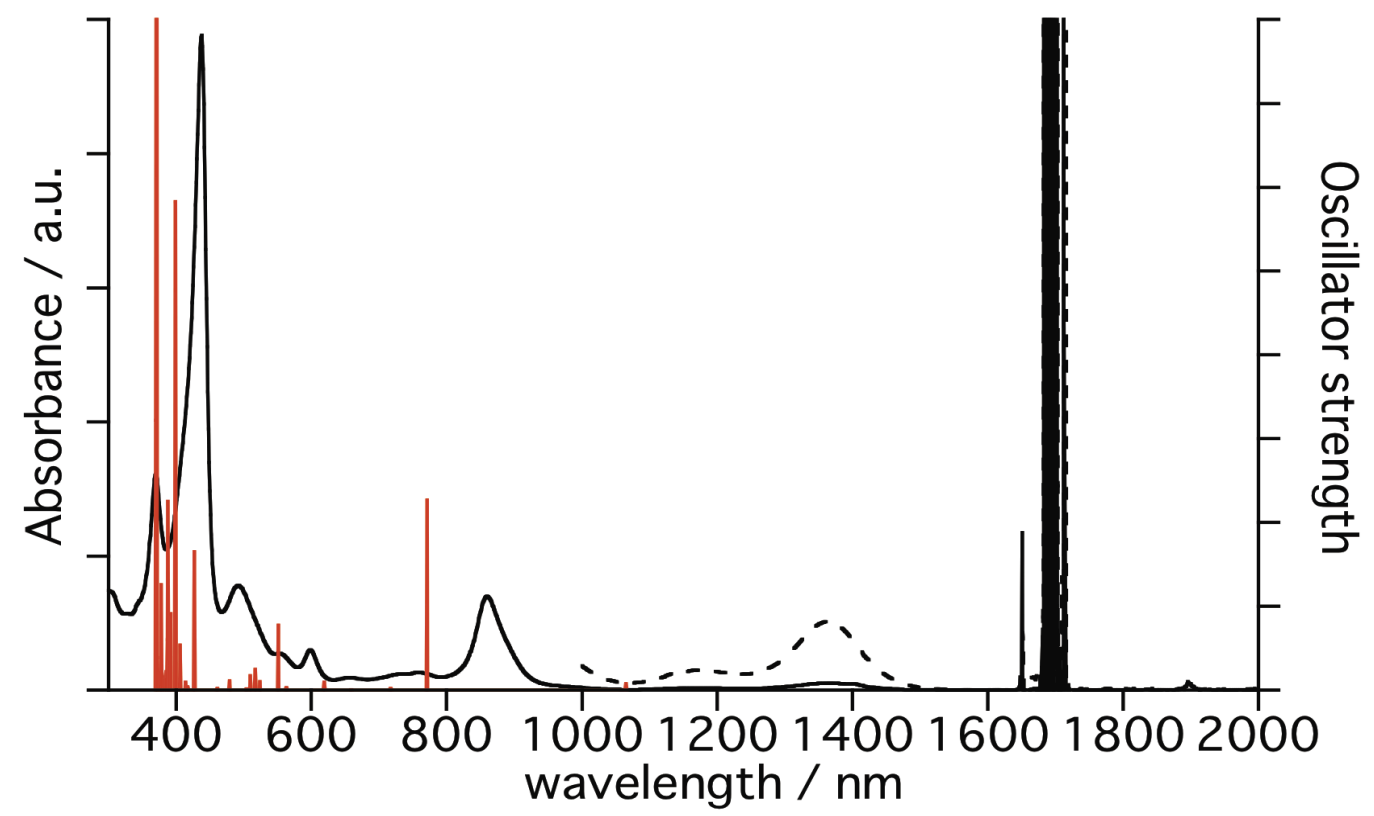

Figure S8-8. UV/Vis/NIR absorption spectrum (black) and oscillator strengths (red) of 8Zn-py calculated at UB3LYP/6-311G(d) (C,H,N,O)-cc-pVQZ (Zn) level. Selected oscillator strengths: $f=$ 0.0038 (1065 nm), 0.1133 (771 nm), 551 (0.0387), 0.2914 (399 nm), 0.1126 (388 nm), 371 (0.4057). 


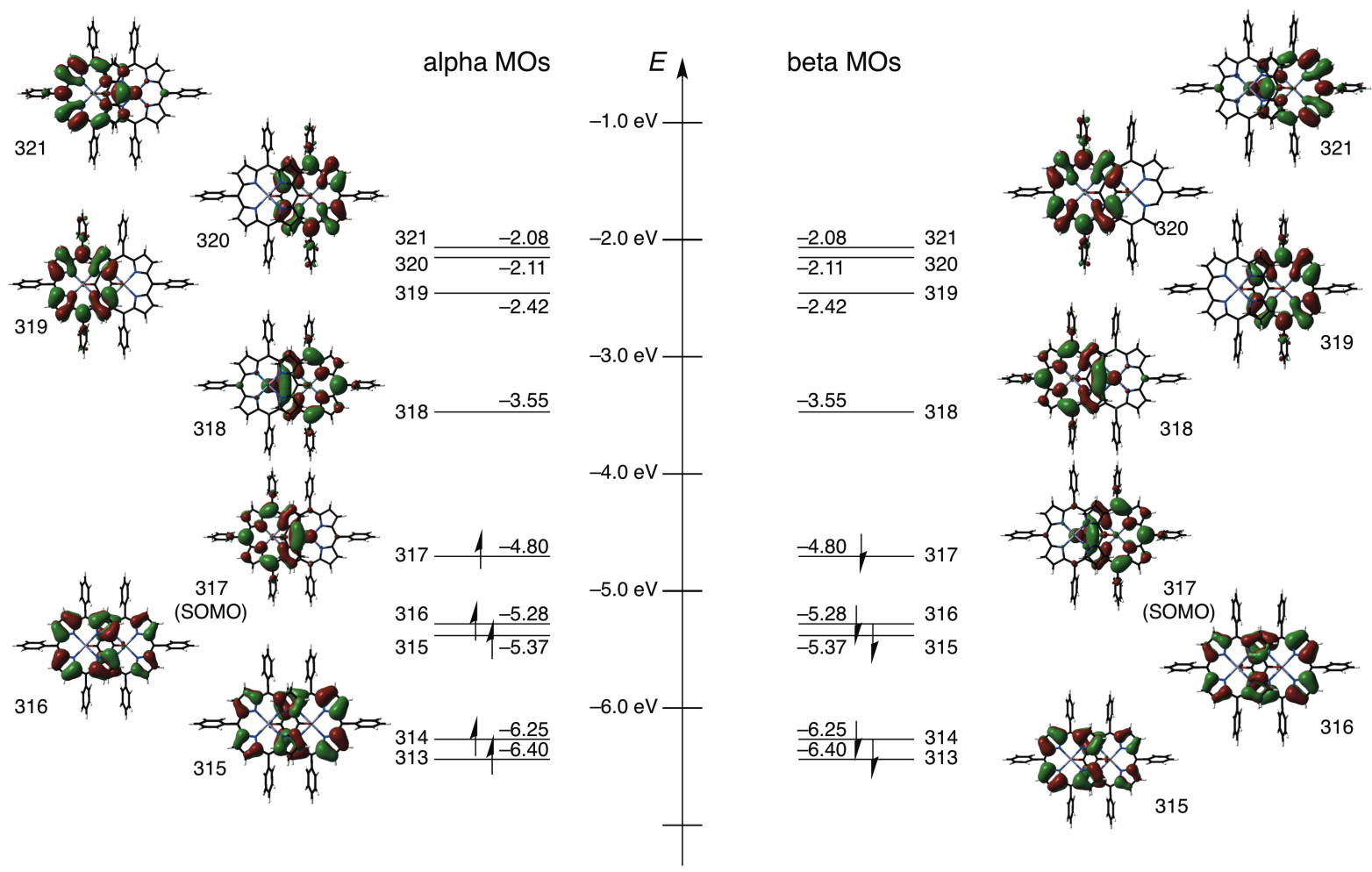

Figure S8-9. Selected molecular orbitals of 9Zn (top view) calculated at UB3LYP/6-311G(d) level.

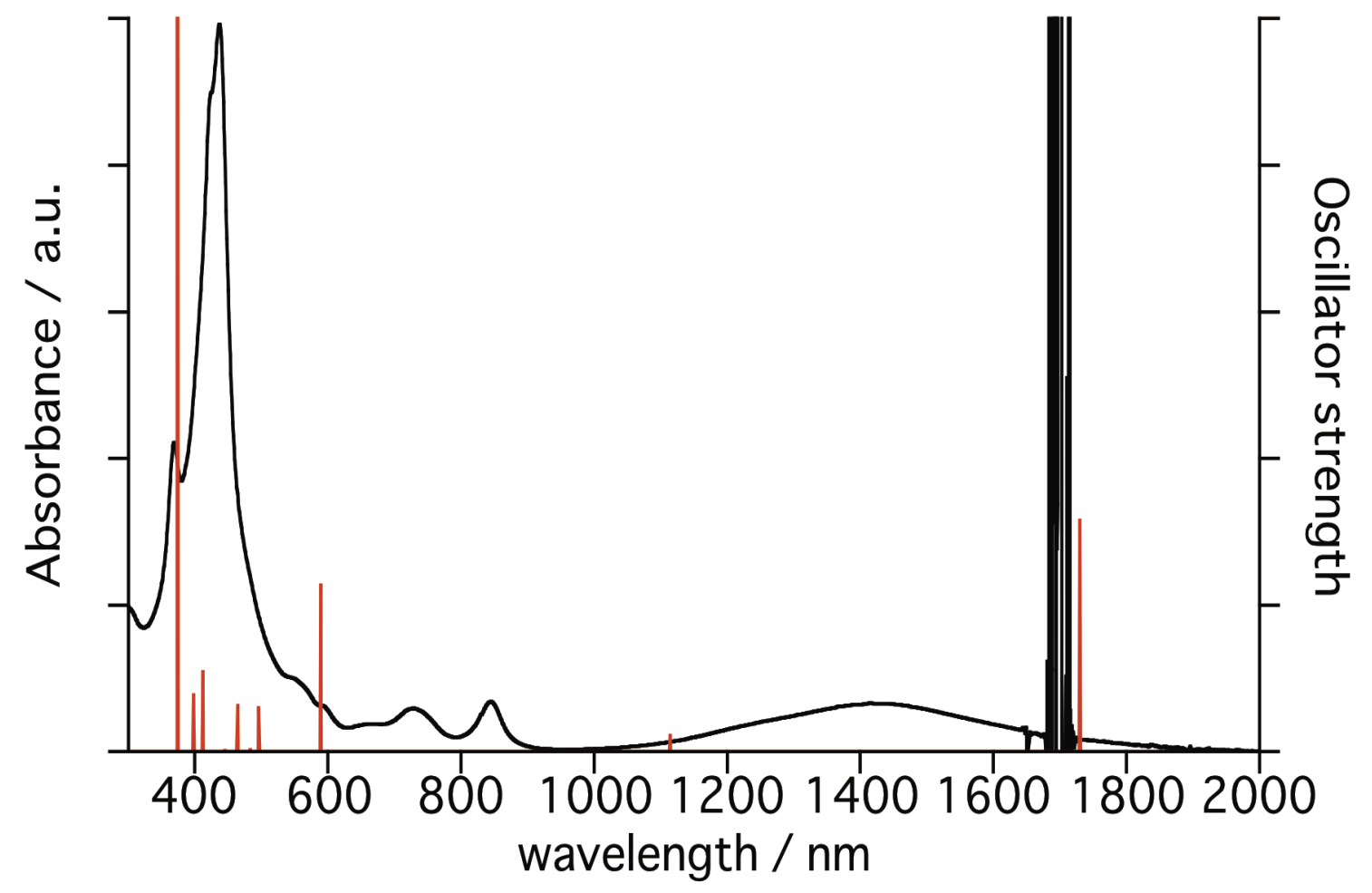

Figure S8-10. UV/Vis/NIR absorption spectrum (black) and oscillator strengths (red) of 9Zn calculated at UCAM-B3LYP/6-311G(d) (C,H,N,O)-cc-pVQZ (Zn) level. Selected oscillator strengths: $f$ $=0.2623(1730 \mathrm{~nm}), 0.0187(1114 \mathrm{~nm}), 0.1891(589 \mathrm{~nm}), 1.3687(374 \mathrm{~nm})$. 


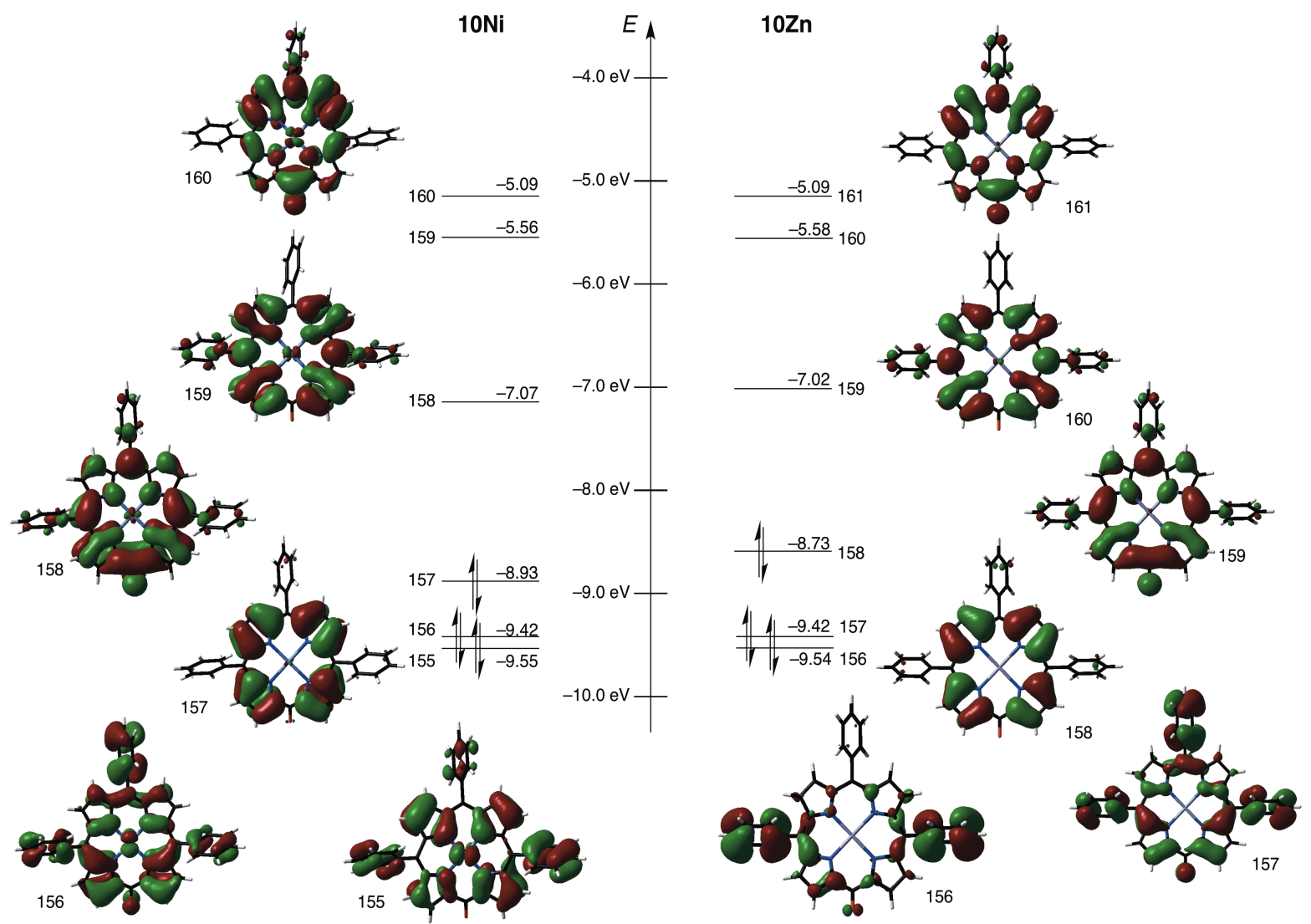

Figure S8-11. Selected molecular orbitals of 10Ni and 10Zn (top view) calculated at UB3LYP/6-311G(d)-cc-pVQZ (Ni, Zn) level. 


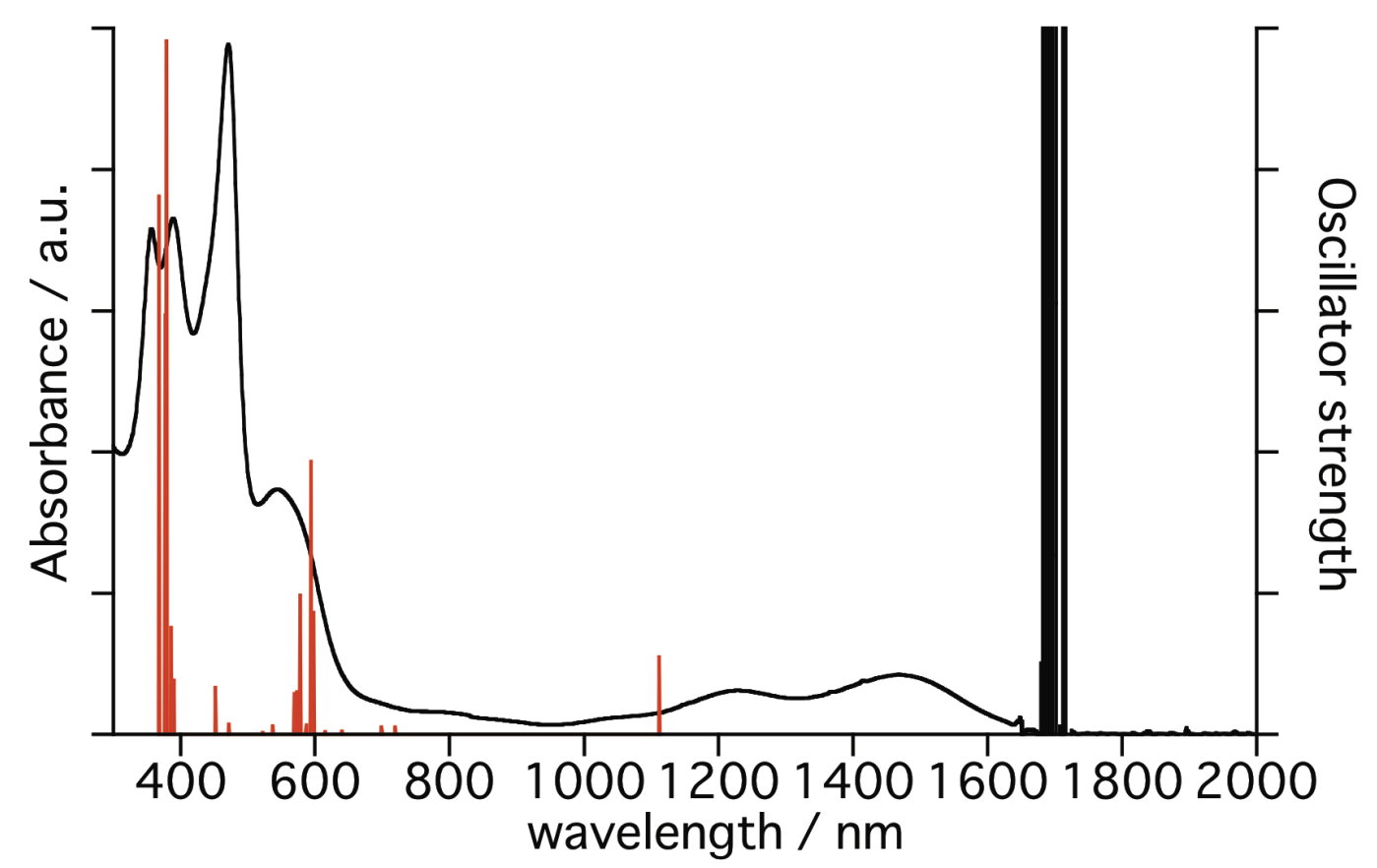

Figure S8-12. UV/Vis/NIR absorption spectrum (black) and oscillator strengths (red) of 10Ni calculated at UB3LYP/6-311G(d) (C,H,N,O)-cc-pVQZ (Zn) level. Selected oscillator strengths: $f=$ $0.0374(1113 \mathrm{~nm}), 0.1314$ (594 nm), 0.3338 (379 nm), 0.2590 (368 nm).

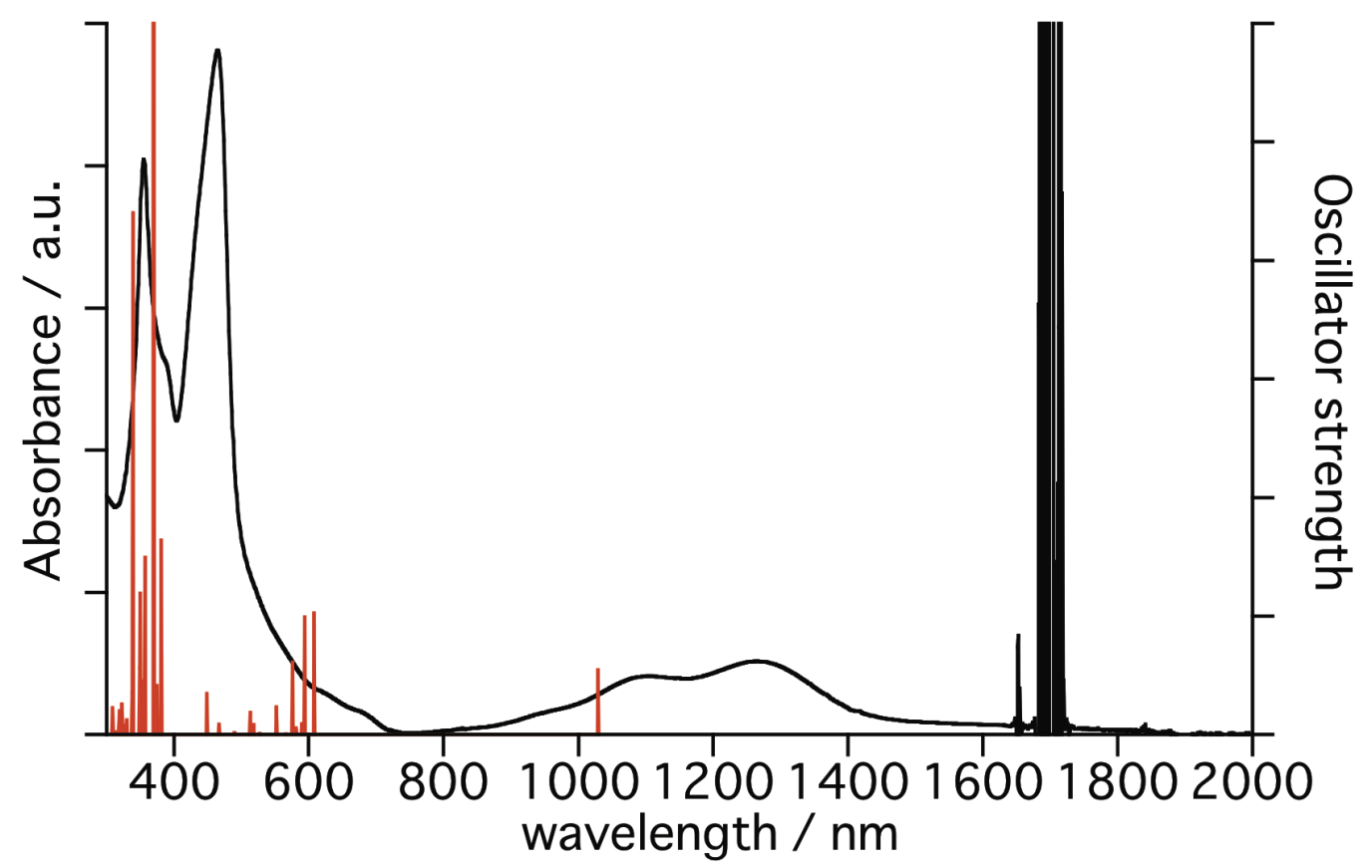

Figure S8-13. UV/Vis/NIR absorption spectrum (black) and oscillator strengths (red) of 1oZn calculated at UB3LYP/6-311G(d) (C,H,N,O)-cc-pVQZ (Zn) level. Selected oscillator strengths: $f=$ $0.0543(1029 \mathrm{~nm}), 0.1025(608 \mathrm{~nm}), 0.0993(594 \mathrm{~nm}), 0.6237(370 \mathrm{~nm}), 0.4401(339 \mathrm{~nm})$. 


\section{Time-resolved TA Spectra and Fluorescence Decay}

The TA spectra were measured by home-made femtosecond time-resolved transient absorption (fs-TA) spectrometer. The spectrometer consists of an optical parametric amplifier (OPA; Palitra, Quantronix) pumped by a Ti:sapphire regenerative amplifier system (Integra-C, Quantronix) operating at $1 \mathrm{kHz}$ repetition rate and an optical detection system. ${ }^{\mathrm{S} 3}$ For time-resolved fluorescence decay profiles, a time-correlated single-photon-counting (TCSPC) system was used for measurements of spontaneous fluorescence decay. $\mathrm{S}_{3}$
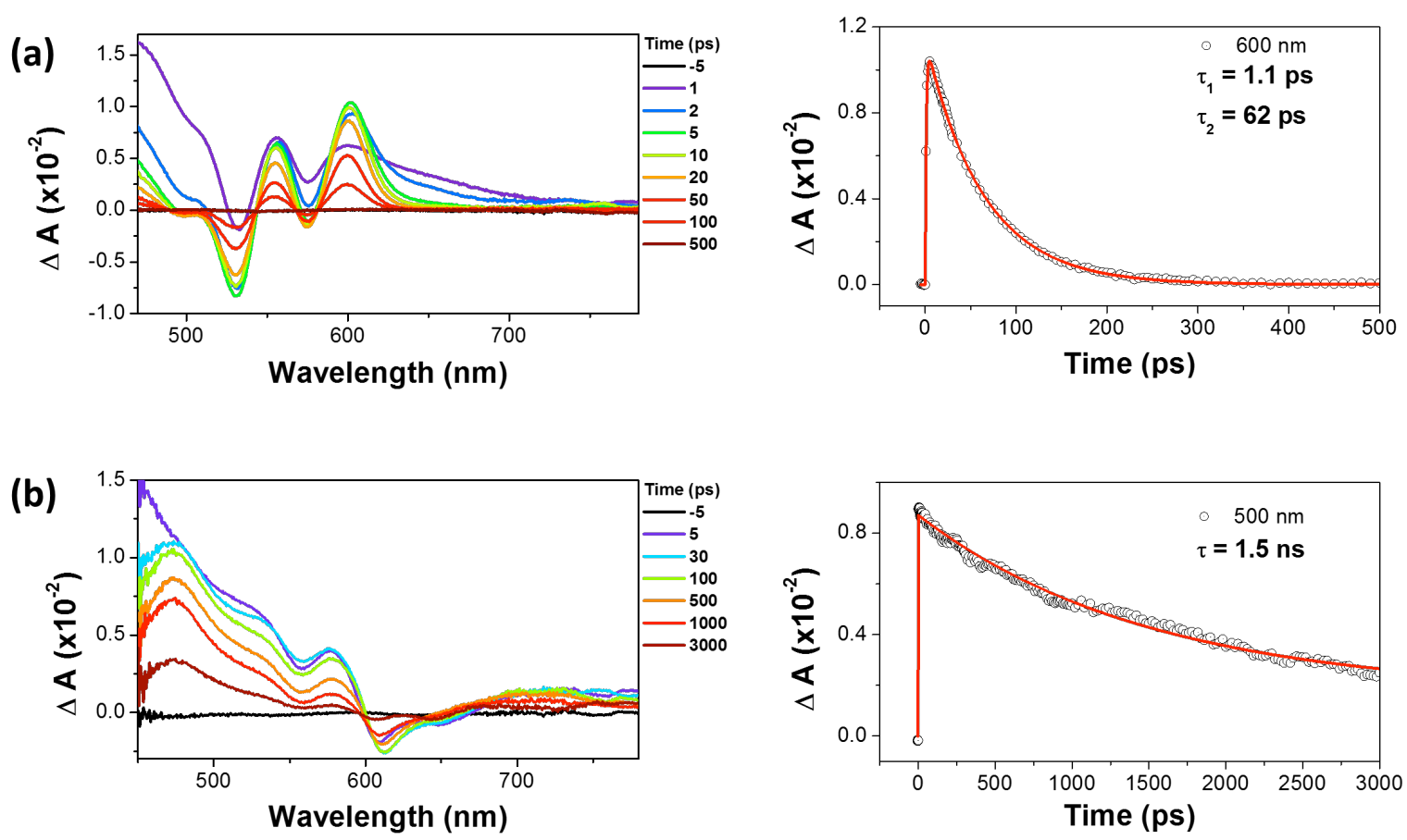

Figure S9-1. TA spectra (left) and decay profiles (right) of (a) $7 \mathbf{N i}$ and (b) $7 \mathbf{Z n}$ in $\mathrm{CH}_{2} \mathrm{Cl}_{2}$ with photoexcitation at $400 \mathrm{~nm}$. 

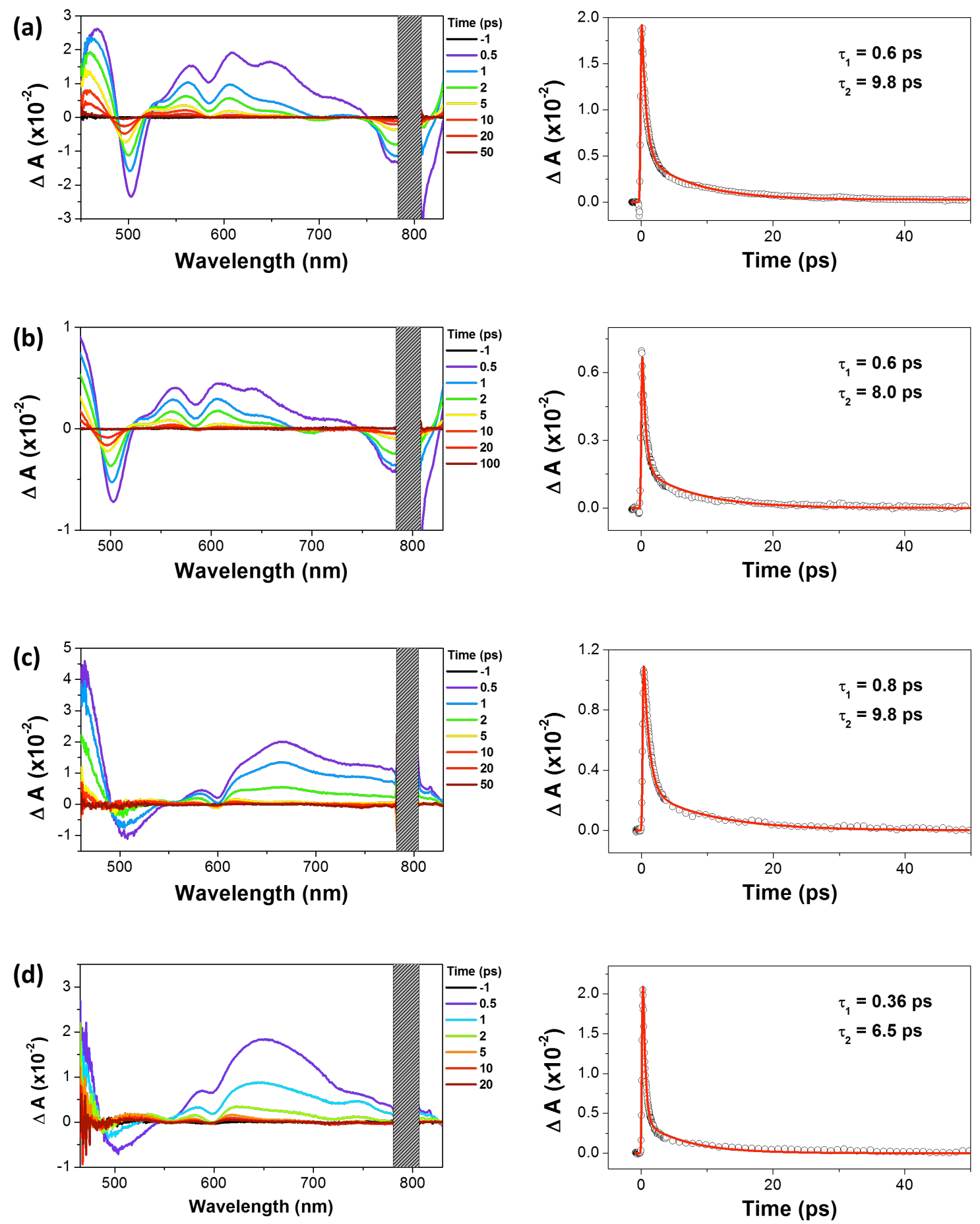

Figure S9-2. TA spectra (left) and decay profiles (right) of (a) $\mathbf{8} \mathbf{H}_{2}$ in $\mathrm{CH}_{2} \mathrm{Cl}_{2}$, (b) $\mathbf{8 N i}$ in $\mathrm{CH}_{2} \mathrm{Cl}_{2}$, (c) 8Zn-py in pyridine and (d) $9 \mathrm{Zn}$ in $\mathrm{CH}_{2} \mathrm{Cl}_{2}$ taken for photoexcitation at $800 \mathrm{~nm}$. TA decay profiles were obtained by monitoring at $570 \mathrm{~nm}$ for $\mathbf{8 H _ { 2 }}, 565 \mathrm{~nm}$ for $\mathbf{8 N i}$, and $615 \mathrm{~nm}$ for $\mathbf{8 Z n}$-py and $\mathbf{9 Z n}$. 

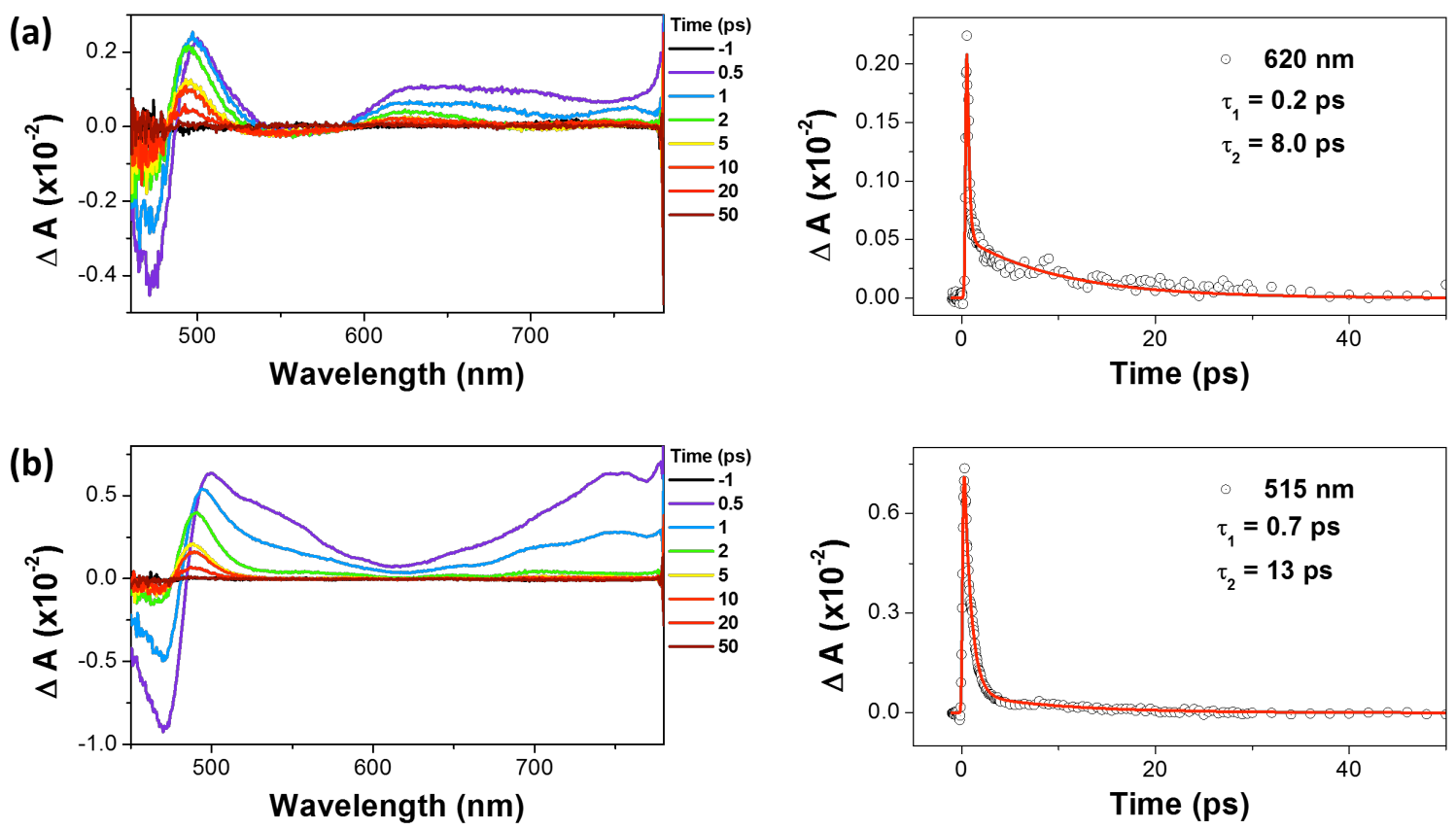

Figure S9-3. TA spectra (left) and decay profiles (right) of (a) $10 \mathrm{Ni}$ and (b) $\mathbf{1 0 Z n}$ in $\mathrm{CH}_{2} \mathrm{Cl}_{2}$ for photoexcitation at $1240 \mathrm{~nm}$. 


\section{Supporting References}

(S1) Altomare, A.; Burla, M. C.; Camalli, M.; Cascarano, G. L.; Gia-co-vazzo, C.; Guagliardi, A.; Moliterni, A. G. G.; Polidori, G.; Spagna, R. J. Appl. Crystallogr. 1999, 32, 115.

(S2) SHELXL-97, Program for the Refinement of Crystal Structures from Diffraction Data, University of Göttingen, Göttingen (Germany); Sheldrick, G. M.; Schneider, T. R.; Methods Enzymol. 1997, 277, 319.

(S3) Shimizu, D.; Oh, J.; Furukawa, K.; Kim, D.; Osuka, A. Angew. Chem., Int. Ed. 2015, 54, 6613. 


\section{Optimized Molecular Geometries}

Cartesian Coordinates of $\mathbf{8} \mathbf{H}_{\mathbf{2}}$ (6-311G(d) Basis set)

UB3LYP (doublet): $E_{\text {total }}=-1757.66887303$ Hartree

\begin{tabular}{|c|c|c|c|c|}
\hline 1 & C & 2.854355 & -2.056187 & -0.007758 \\
\hline 2 & $\mathrm{C}$ & 2.472202 & -4.290606 & -0.059036 \\
\hline 3 & $\mathrm{C}$ & -2.598586 & -4.249376 & -0.043154 \\
\hline 4 & C & 1.239404 & -3.615541 & 0.016769 \\
\hline 5 & $\mathrm{C}$ & 3.4704 & -3.335381 & -0.080266 \\
\hline 6 & $\mathrm{C}$ & 2.82513 & 0.451444 & -0.003659 \\
\hline 7 & $\mathrm{C}$ & 0.044421 & 2.657854 & 0.025257 \\
\hline 8 & $\mathrm{C}$ & -1.227951 & 2.052337 & 0.007682 \\
\hline 9 & $\mathrm{C}$ & 0.090746 & 6.962074 & 0.139485 \\
\hline 10 & $\mathrm{C}$ & -2.853711 & 0.486378 & -0.021244 \\
\hline 11 & $\mathrm{C}$ & -2.871547 & -2.015183 & -0.016906 \\
\hline 12 & $\mathrm{C}$ & 0.058984 & 4.157868 & 0.065627 \\
\hline 13 & $\mathrm{C}$ & -1.338051 & -3.540108 & 0.012534 \\
\hline 14 & $\mathrm{C}$ & -3.560543 & -3.293579 & -0.067273 \\
\hline 15 & $\mathrm{C}$ & -2.487058 & 2.720235 & 0.044781 \\
\hline 16 & $\mathrm{C}$ & 7.786938 & -0.817306 & -0.068874 \\
\hline 17 & $\mathrm{C}$ & -0.064832 & -4.28188 & 0.032539 \\
\hline 18 & $\mathrm{C}$ & -7.085196 & -0.298834 & -1.2035 \\
\hline 19 & $\mathrm{C}$ & 2.56303 & 2.686116 & 0.076337 \\
\hline 20 & $\mathrm{C}$ & 3.48472 & -0.7915 & -0.013404 \\
\hline 21 & $\mathrm{C}$ & 0.187305 & 6.292677 & -1.07874 \\
\hline 22 & $\mathrm{C}$ & -5.731306 & -1.1294 & 1.082436 \\
\hline 23 & $\mathrm{C}$ & 7.074829 & -0.360439 & -1.176052 \\
\hline 24 & $\mathrm{C}$ & 1.285011 & 1.988886 & 0.017738 \\
\hline 25 & $\mathrm{C}$ & -5.692652 & -0.323986 & -1.188917 \\
\hline 26 & $\mathrm{C}$ & -0.022351 & 6.23185 & 1.320811 \\
\hline 27 & $\mathrm{C}$ & -7.804555 & -0.682707 & -0.073397 \\
\hline 28 & $\mathrm{C}$ & 0.172157 & 4.899672 & -1.115206 \\
\hline 29 & $\mathrm{C}$ & -3.475861 & 1.765571 & 0.032426 \\
\hline 30 & $\mathrm{C}$ & $5 \cdot 705737$ & -1.262515 & 1.072881 \\
\hline 31 & $\mathrm{C}$ & -3.502319 & -0.774512 & -0.030697 \\
\hline
\end{tabular}




\begin{tabular}{|c|c|c|c|c|}
\hline 32 & $\mathrm{C}$ & $3 \cdot 519747$ & 1.730832 & 0.066462 \\
\hline 33 & $\mathrm{C}$ & 7.098308 & -1.266181 & 1.05634 \\
\hline 34 & $\mathrm{C}$ & 5.682293 & -0.347595 & -1.156688 \\
\hline 35 & $\mathrm{C}$ & -7.12385 & -1.096252 & 1.069833 \\
\hline 36 & $\mathrm{C}$ & -0.039783 & 4.838873 & 1.283939 \\
\hline 37 & $\mathrm{C}$ & -4.999698 & -0.742568 & -0.046604 \\
\hline 38 & $\mathrm{C}$ & 4.979999 & -0.799084 & -0.032042 \\
\hline 39 & $\mathrm{H}$ & 2.567781 & $-5 \cdot 363758$ & -0.102131 \\
\hline 40 & $\mathrm{H}$ & -2.701374 & $-5 \cdot 322966$ & -0.068719 \\
\hline 41 & $\mathrm{H}$ & 4.532833 & -3.50428 & -0.15341 \\
\hline 42 & $\mathrm{H}$ & 0.104022 & 8.046852 & 0.16819 \\
\hline 43 & $\mathrm{H}$ & -4.629166 & -3.43217 & -0.126217 \\
\hline 44 & $\mathrm{H}$ & -2.613508 & 3.790375 & 0.083574 \\
\hline 45 & $\mathrm{H}$ & 8.872025 & -0.824148 & -0.083033 \\
\hline 46 & $\mathrm{H}$ & 2.697479 & 3.755663 & 0.129819 \\
\hline 47 & $\mathrm{H}$ & -5.203163 & -1.451226 & 1.973651 \\
\hline 48 & $\mathrm{H}$ & -5.135688 & -0.030146 & -2.072538 \\
\hline 49 & $\mathrm{H}$ & -8.889478 & -0.660946 & -0.084242 \\
\hline 50 & $\mathrm{H}$ & 0.248899 & 4.380292 & -2.064926 \\
\hline 51 & $\mathrm{H}$ & -4.540665 & 1.930651 & 0.067754 \\
\hline 52 & $\mathrm{H}$ & 5.172988 & -1.609151 & 1.952041 \\
\hline 53 & $\mathrm{H}$ & 4.588722 & 1.868369 & 0.118559 \\
\hline 54 & $\mathrm{H}$ & 5.130706 & 0.004398 & -2.021992 \\
\hline 55 & $\mathrm{H}$ & -0.125601 & 4.272057 & 2.205358 \\
\hline 56 & $\mathrm{H}$ & -0.800252 & -0.026128 & -0.067657 \\
\hline 57 & $\mathrm{H}$ & 0.799894 & -1.543706 & 0.091339 \\
\hline 58 & $\mathrm{~N}$ & 1.469449 & 0.63809 & -0.026467 \\
\hline 59 & $\mathrm{~N}$ & -1.493009 & -2.216781 & 0.026566 \\
\hline 60 & $\mathrm{~N}$ & -1.498189 & 0.705477 & -0.035038 \\
\hline 61 & $\mathrm{~N}$ & 1.49472 & -2.277753 & 0.047238 \\
\hline 62 & $\mathrm{O}$ & -0.090672 & -5.51551 & 0.041833 \\
\hline 63 & $\mathrm{H}$ & -7.607711 & 0.018057 & -2.100406 \\
\hline 64 & $\mathrm{H}$ & -7.676365 & -1.394655 & 1.954998 \\
\hline 65 & $\mathrm{H}$ & 7.603094 & -0.014712 & -2.058837 \\
\hline
\end{tabular}




$\begin{array}{rrrrr}66 & \mathrm{H} & 7.644987 & -1.619431 & 1.924808 \\ 67 & \mathrm{H} & 0.275357 & 6.854118 & -2.003425 \\ 68 & \mathrm{H} & -0.096426 & 6.745754 & 2.27393\end{array}$

Cartesian Coordinates of 8Ni (6-311G(d)(C,H,N,O)-cc-pVQZ(Ni) Basis sets) UB3LYP (doublet): $E_{\text {total }}=-3265.00134387$ Hartree

\begin{tabular}{|c|c|c|c|c|}
\hline 1 & $\mathrm{~N}$ & 1.374952 & 0.67905 & -0.050454 \\
\hline 2 & $\mathrm{~N}$ & -1.399212 & -2.039957 & -0.439446 \\
\hline 3 & $\mathrm{~N}$ & 1.378712 & -2.045635 & -0.447018 \\
\hline 4 & $\mathrm{O}$ & -0.022094 & -5.040876 & -1.860399 \\
\hline 5 & $\mathrm{C}$ & 2.719398 & -1.94285 & -0.086659 \\
\hline 6 & $\mathrm{~N}$ & -1.381939 & 0.691587 & -0.053904 \\
\hline 7 & $\mathrm{C}$ & 2.704781 & 0.478589 & 0.241516 \\
\hline 8 & $\mathrm{C}$ & 1.221686 & 2.041868 & -0.165036 \\
\hline 9 & $\mathrm{C}$ & 1.233854 & $-3 \cdot 310177$ & -0.910449 \\
\hline 10 & $\mathrm{C}$ & 2.478509 & 2.703549 & 0.043739 \\
\hline 11 & $\mathrm{H}$ & 2.630325 & 3.770974 & 0.0081 \\
\hline 12 & $\mathrm{C}$ & -4.812928 & -0.76037 & 0.657369 \\
\hline 13 & $\mathrm{C}$ & 4.788381 & -0.801886 & 0.698499 \\
\hline 14 & $\mathrm{C}$ & 3.391079 & 1.735399 & 0.326556 \\
\hline 15 & $\mathrm{H}$ & 4.440729 & 1.854607 & 0.545279 \\
\hline 16 & $\mathrm{C}$ & -3.368898 & -0.743659 & 0.274642 \\
\hline 17 & $\mathrm{C}$ & -1.256426 & -3.312604 & -0.879736 \\
\hline 18 & $\mathrm{C}$ & -1.223122 & 2.052811 & -0.20454 \\
\hline 19 & $\mathrm{C}$ & $3 \cdot 34789$ & -0.765717 & 0.299854 \\
\hline 20 & $\mathrm{C}$ & 0.003698 & 2.712361 & -0.32188 \\
\hline 21 & $\mathrm{C}$ & -2.722492 & 0.498864 & 0.183074 \\
\hline 22 & $\mathrm{C}$ & 0.103967 & 6.972782 & -0.905032 \\
\hline 23 & $\mathrm{H}$ & 0.133008 & 8.047176 & -1.055141 \\
\hline 24 & $\mathrm{C}$ & -2.736673 & -1.932795 & -0.066259 \\
\hline 25 & $\mathrm{C}$ & 5.797361 & -1.00309 & -0.250976 \\
\hline 26 & $\mathrm{H}$ & $5 \cdot 529125$ & -1.131585 & -1.294302 \\
\hline 27 & $\mathrm{C}$ & 7.484353 & -0.855939 & 1.471377 \\
\hline 28 & $\mathrm{H}$ & 8.527268 & -0.878334 & 1.770596 \\
\hline
\end{tabular}




\begin{tabular}{|c|c|c|c|c|}
\hline 29 & $\mathrm{C}$ & -0.335706 & 5.068637 & 0.514543 \\
\hline 30 & $\mathrm{H}$ & -0.638928 & 4.663325 & 1.474212 \\
\hline 31 & $\mathrm{C}$ & 5.148546 & -0.622367 & 2.039241 \\
\hline 32 & $\mathrm{H}$ & 4.37361 & -0.466584 & 2.782584 \\
\hline 33 & $\mathrm{C}$ & -0.295607 & 6.44775 & 0.322412 \\
\hline 34 & $\mathrm{C}$ & 0.027432 & 4.193807 & -0.517038 \\
\hline 35 & $\mathrm{C}$ & -5.809421 & -1.071464 & -0.276281 \\
\hline 36 & $\mathrm{H}$ & -5.528659 & -1.301929 & -1.298428 \\
\hline 37 & $\mathrm{C}$ & 0.468515 & 6.111295 & -1.937544 \\
\hline 38 & $\mathrm{C}$ & -0.015315 & -3.940759 & -1.29895 \\
\hline 39 & $\mathrm{C}$ & -2.493237 & 2.714247 & -0.104126 \\
\hline 40 & $\mathrm{H}$ & -2.653018 & $3 \cdot 774733$ & -0.218149 \\
\hline 41 & $\mathrm{C}$ & 7.136189 & -1.027593 & 0.133091 \\
\hline 42 & $\mathrm{C}$ & -6.534587 & -0.453229 & 2.339466 \\
\hline 43 & $\mathrm{C}$ & -7.51831 & -0.765624 & 1.403425 \\
\hline 44 & $\mathrm{H}$ & -8.56445 & -0.76902 & 1.691949 \\
\hline 45 & $\mathrm{C}$ & 6.487229 & -0.653764 & 2.423269 \\
\hline 46 & $\mathrm{C}$ & 0.434628 & 4.732172 & -1.743838 \\
\hline 47 & $\mathrm{H}$ & 0.718579 & 4.063491 & -2.549804 \\
\hline 48 & $\mathrm{C}$ & -3.415806 & 1.754794 & 0.175278 \\
\hline 49 & $\mathrm{H}$ & -4.477347 & 1.877585 & 0.322645 \\
\hline 50 & $\mathrm{C}$ & -5.192271 & -0.44495 & 1.967961 \\
\hline 51 & $\mathrm{H}$ & -4.42806 & $-0.20275^{2}$ & 2.699078 \\
\hline 52 & $\mathrm{C}$ & 2.453498 & -4.039059 & -0.854359 \\
\hline 53 & $\mathrm{H}$ & 2.560937 & -5.064749 & -1.170806 \\
\hline 54 & $\mathrm{C}$ & -7.152158 & -1.071218 & 0.09413 \\
\hline 55 & $\mathrm{C}$ & $-3 \cdot 389918$ & -3.198487 & -0.23873 \\
\hline 56 & $\mathrm{H}$ & -4.419902 & -3.404143 & 0.006708 \\
\hline 57 & $\mathrm{C}$ & $3 \cdot 377211$ & -3.198845 & -0.302727 \\
\hline 58 & $\mathrm{H}$ & 4.412811 & -3.402814 & -0.079938 \\
\hline 59 & $\mathrm{C}$ & -2.470749 & -4.046445 & -0.785608 \\
\hline 60 & $\mathrm{H}$ & -2.578464 & -5.079106 & -1.078389 \\
\hline 61 & $\mathrm{H}$ & 0.779892 & 6.511843 & -2.896969 \\
\hline 62 & $\mathrm{H}$ & -0.573756 & 7.112037 & 1.134436 \\
\hline
\end{tabular}




$\begin{array}{lllll}63 & \mathrm{H} & 6.750042 & -0.521387 & 3.467904 \\ 64 & \mathrm{H} & 7.907286 & -1.179592 & -0.615374 \\ 65 & \mathrm{H} & -6.810801 & -0.216076 & 3.361872 \\ 66 & \mathrm{H} & -7.912652 & -1.307964 & -0.643079 \\ 67 & \mathrm{Ni} & -0.006996 & -0.676937 & -0.253717\end{array}$

Cartesian Coordinates of 8Zn (6-311G(d)(C,H,N,O)-cc-pVQZ(Zn) Basis sets) UB3LYP (doublet): $E_{\text {total }}=-3536.05211477$ Hartree

\begin{tabular}{|c|c|c|c|c|}
\hline 1 & $\mathrm{~N}$ & 1.456281 & 0.739289 & 0.002053 \\
\hline 2 & $\mathrm{~N}$ & -1.461435 & -2.17544 & -0.015287 \\
\hline 3 & $\mathrm{~N}$ & 1.469587 & -2.170586 & -0.013619 \\
\hline 4 & $\mathrm{O}$ & 0.010232 & -5.458986 & -0.004819 \\
\hline 5 & $\mathrm{C}$ & 2.842429 & -1.960593 & 0.008689 \\
\hline 6 & $\mathrm{~N}$ & -1.458422 & 0.73462 & 0.034645 \\
\hline 7 & $\mathrm{C}$ & 2.816151 & 0.542271 & 0.020259 \\
\hline 8 & $\mathrm{C}$ & 1.250498 & 2.098039 & -0.006222 \\
\hline 9 & $\mathrm{C}$ & 1.290795 & -3.511359 & -0.002871 \\
\hline 10 & $\mathrm{C}$ & 2.520898 & 2.776575 & 0.014183 \\
\hline 11 & $\mathrm{H}$ & 2.660044 & 3.846241 & 0.024984 \\
\hline 12 & $\mathrm{C}$ & -4.965859 & -0.711936 & 0.011842 \\
\hline 13 & $\mathrm{C}$ & 4.969249 & -0.69634 & 0.041446 \\
\hline 14 & $\mathrm{C}$ & 3.486571 & 1.815591 & 0.02126 \\
\hline 15 & $\mathrm{H}$ & 4.555599 & 1.960472 & 0.02555 \\
\hline 16 & $\mathrm{C}$ & -3.468829 & -0.721369 & 0.011347 \\
\hline 17 & $\mathrm{C}$ & -1.276489 & -3.515392 & 0.011823 \\
\hline 18 & $\mathrm{C}$ & -1.255726 & 2.092864 & -0.021064 \\
\hline 19 & $\mathrm{C}$ & 3.470952 & -0.709322 & 0.022778 \\
\hline 20 & $\mathrm{C}$ & -0.003743 & 2.741971 & -0.024091 \\
\hline 21 & $\mathrm{C}$ & -2.817152 & $0.53255^{8}$ & -0.004252 \\
\hline 22 & $\mathrm{C}$ & -0.019456 & 7.04661 & -0.113337 \\
\hline 23 & $\mathrm{H}$ & -0.023866 & 8.131593 & -0.135697 \\
\hline 24 & $\mathrm{C}$ & -2.834825 & -1.970782 & 0.019397 \\
\hline 25 & $\mathrm{C}$ & 5.698032 & -1.030437 & -1.105901 \\
\hline 26 & $\mathrm{H}$ & 5.167814 & -1.2958 & -2.01453 \\
\hline
\end{tabular}




\begin{tabular}{|c|c|c|c|c|}
\hline 27 & $\mathrm{C}$ & $7 \cdot 774942$ & -0.676223 & 0.075242 \\
\hline 28 & $\mathrm{H}$ & 8.860049 & -0.669085 & 0.088514 \\
\hline 29 & $\mathrm{C}$ & -0.406754 & 4.975271 & 1.067115 \\
\hline 30 & $\mathrm{H}$ & -0.708023 & 4.450121 & 1.967577 \\
\hline 31 & $\mathrm{C}$ & 5.665374 & -0.352959 & 1.206302 \\
\hline 32 & $\mathrm{H}$ & 5.110125 & -0.0996 & 2.103456 \\
\hline 33 & $\mathrm{C}$ & -0.409324 & 6.368016 & 1.039559 \\
\hline 34 & C & -0.007914 & 4.239093 & -0.055378 \\
\hline 35 & $\mathrm{C}$ & -5.682438 & -1.144342 & -1.110818 \\
\hline 36 & $\mathrm{H}$ & -5.142124 & -1.483296 & -1.988357 \\
\hline 37 & $\mathrm{C}$ & 0.375873 & 6.324253 & -1.237454 \\
\hline 38 & $\mathrm{C}$ & 0.008313 & -4.222543 & -0.00338 \\
\hline 39 & $\mathrm{C}$ & -2.526286 & 2.76521 & -0.10853 \\
\hline 40 & $\mathrm{H}$ & -2.666939 & 3.831916 & -0.186375 \\
\hline 41 & $\mathrm{C}$ & 7.09103 & -1.017011 & -1.089872 \\
\hline 42 & $\mathrm{C}$ & -7.069258 & -0.267629 & 1.137269 \\
\hline 43 & $\mathrm{C}$ & -7.772698 & -0.695372 & 0.012961 \\
\hline 44 & $\mathrm{H}$ & -8.857891 & -0.689551 & 0.013634 \\
\hline 45 & $\mathrm{C}$ & 7.058296 & -0.346119 & 1.223816 \\
\hline 46 & $\mathrm{C}$ & 0.384576 & 4.931581 & -1.207569 \\
\hline 47 & $\mathrm{H}$ & o.689189 & 4.372315 & -2.086091 \\
\hline 48 & $\mathrm{C}$ & -3.489317 & 1.801593 & -0.097837 \\
\hline 49 & $\mathrm{H}$ & -4.556723 & 1.9418 & -0.16635 \\
\hline 50 & $\mathrm{C}$ & -5.676471 & -0.271403 & 1.13521 \\
\hline 51 & $\mathrm{H}$ & -5.131956 & 0.058514 & 2.013767 \\
\hline 52 & $\mathrm{C}$ & 2.54061 & -4.19708 & 0.02691 \\
\hline 53 & $\mathrm{H}$ & 2.648398 & -5.270227 & 0.044364 \\
\hline 54 & $\mathrm{C}$ & -7.075282 & -1.13163 & -1.111671 \\
\hline 55 & $\mathrm{C}$ & -3.496963 & -3.246207 & 0.079689 \\
\hline 56 & $\mathrm{H}$ & -4.564202 & -3.394705 & 0.135286 \\
\hline 57 & $\mathrm{C}$ & $3 \cdot 511016$ & -3.233674 & 0.03965 \\
\hline 58 & $\mathrm{H}$ & 4.579841 & $-3 \cdot 377332$ & 0.075926 \\
\hline 59 & $\mathrm{C}$ & -2.522485 & -4.205606 & 0.069014 \\
\hline 60 & $\mathrm{H}$ & -2.625477 & -5.278803 & 0.103651 \\
\hline
\end{tabular}




$\begin{array}{rlrrr}61 & \mathrm{H} & 0.676385 & 6.84448 & -2.141376 \\ 62 & \mathrm{H} & -0.714655 & 6.922655 & 1.921153 \\ 63 & \mathrm{H} & 7.583083 & -0.085201 & 2.13732 \\ 64 & \mathrm{H} & 7.6413 & -1.273337 & -1.989567 \\ 65 & \mathrm{H} & -7.604543 & 0.067905 & 2.01979 \\ 66 & \mathrm{H} & -7.615082 & -1.463193 & -1.992905 \\ 67 & \mathrm{Zn} & 0.001523 & -0.718939 & 0.00124\end{array}$

Cartesian Coordinates of 8Zn-py (6-311G(d)(C,H,N,O)-cc-pVQZ(Zn) Basis sets) UB3LYP (doublet): $E_{\text {total }}=-3784.41057687$ Hartree

\begin{tabular}{|c|c|c|c|c|}
\hline 1 & $\mathrm{Zn}$ & -0.016093 & -0.6187 & -0.113431 \\
\hline 2 & $\mathrm{O}$ & -0.093512 & $-5 \cdot 328318$ & -0.713504 \\
\hline 3 & $\mathrm{~N}$ & 1.430941 & -2.076703 & -0.553035 \\
\hline 4 & $\mathrm{~N}$ & -1.509089 & -2.025985 & -0.544682 \\
\hline 5 & $\mathrm{~N}$ & 1.478434 & 0.837657 & -0.294189 \\
\hline 6 & $\mathrm{~N}$ & -0.02536 & -0.826397 & 2.109211 \\
\hline 7 & $\mathrm{~N}$ & -1.450635 & 0.890025 & -0.341714 \\
\hline 8 & $\mathrm{C}$ & 4.950066 & -0.656214 & -0.599287 \\
\hline 9 & $\mathrm{C}$ & -4.977455 & -0.483898 & -0.55808 \\
\hline 10 & $\mathrm{C}$ & 2.805194 & -1.890691 & -0.564393 \\
\hline 11 & $\mathrm{C}$ & -2.484533 & 2.942549 & -0.278369 \\
\hline 12 & $\mathrm{H}$ & -2.608411 & 4.013094 & -0.228574 \\
\hline 13 & $\mathrm{C}$ & 0.076831 & $4 \cdot 358545$ & -0.217712 \\
\hline 14 & $\mathrm{C}$ & 1.291707 & 2.196513 & -0.304968 \\
\hline 15 & $\mathrm{C}$ & 3.452903 & -3.176215 & -0.637084 \\
\hline 16 & $\mathrm{H}$ & 4.519189 & $-3 \cdot 341813$ & -0.64864 \\
\hline 17 & $\mathrm{C}$ & 0.045114 & 2.86188 & -0.256859 \\
\hline 18 & $\mathrm{C}$ & 2.826707 & 0.616767 & -0.424702 \\
\hline 19 & $\mathrm{C}$ & -3.463453 & 2.000224 & -0.357298 \\
\hline 20 & $\mathrm{H}$ & -4.529063 & 2.164569 & -0.397414 \\
\hline 21 & $\mathrm{C}$ & 2.464392 & -4.118748 & -0.680471 \\
\hline 22 & $\mathrm{H}$ & 2.55169 & -5.192274 & -0.74205 \\
\hline 23 & $\mathrm{C}$ & -1.222767 & 2.239626 & -0.283915 \\
\hline 24 & $\mathrm{C}$ & 1.225742 & -3.406322 & -0.627616 \\
\hline
\end{tabular}




\begin{tabular}{|c|c|c|c|c|}
\hline 25 & $\mathrm{C}$ & 3.514609 & 1.879986 & -0.511909 \\
\hline 26 & $\mathrm{H}$ & 4.57764 & 2.008846 & -0.644542 \\
\hline 27 & $\mathrm{C}$ & 6.985165 & -1.016967 & -1.87209 \\
\hline 28 & $\mathrm{C}$ & -7.124614 & -0.148621 & 0.52263 \\
\hline 29 & $\mathrm{C}$ & $5 \cdot 594739$ & -1.017777 & -1.788737 \\
\hline 30 & $\mathrm{H}$ & 4.99899 & -1.291859 & -2.652868 \\
\hline 31 & $\mathrm{C}$ & $5 \cdot 73224$ & -0.297698 & 0.505379 \\
\hline 32 & $\mathrm{H}$ & 5.244893 & -0.021073 & 1.434552 \\
\hline 33 & $\mathrm{C}$ & 0.538571 & 6.425091 & 0.972274 \\
\hline 34 & $\mathrm{C}$ & 2.5671 & 2.856783 & -0.436062 \\
\hline 35 & $\mathrm{H}$ & 2.719309 & 3.923325 & -0.495623 \\
\hline 36 & $\mathrm{C}$ & -7.78137 & -0.402075 & -0.679794 \\
\hline 37 & $\mathrm{H}$ & -8.865184 & -0.370608 & -0.726864 \\
\hline 38 & $\mathrm{C}$ & -2.609649 & -4.02634 & -0.738847 \\
\hline 39 & $\mathrm{H}$ & -2.735962 & -5.094104 & -0.828317 \\
\hline 40 & $\mathrm{C}$ & -5.647618 & -0.740614 & -1.760154 \\
\hline 41 & $\mathrm{H}$ & -5.071554 & -0.968585 & -2.65074 \\
\hline 42 & $\mathrm{C}$ & $-5 \cdot 733144$ & -0.186182 & 0.582104 \\
\hline 43 & $\mathrm{H}$ & -5.22502 & 0.011376 & 1.520364 \\
\hline 44 & $\mathrm{C}$ & 0.506215 & 5.033017 & 0.932064 \\
\hline 45 & $\mathrm{H}$ & 0.808924 & 4.459099 & 1.801882 \\
\hline 46 & $\mathrm{C}$ & -0.275643 & 6.507247 & -1.29287 \\
\hline 47 & $\mathrm{C}$ & 0.808994 & -0.094283 & 2.860916 \\
\hline 48 & $\mathrm{H}$ & 1.449391 & 0.593996 & 2.321077 \\
\hline 49 & $\mathrm{C}$ & -0.073513 & -4.093167 & -0.660782 \\
\hline 50 & $\mathrm{C}$ & -1.348521 & -3.363465 & -0.646907 \\
\hline 51 & $\mathrm{C}$ & -2.809446 & 0.714341 & -0.392442 \\
\hline 52 & $\mathrm{C}$ & 7.122842 & -0.305637 & 0.425084 \\
\hline 53 & $\mathrm{C}$ & -7.038617 & -0.696833 & -1.821281 \\
\hline 54 & $\mathrm{C}$ & -2.874536 & -1.790121 & -0.57693 \\
\hline 55 & $\mathrm{C}$ & -0.313708 & 5.115273 & -1.329869 \\
\hline 56 & $\mathrm{H}$ & -0.639491 & 4.605463 & -2.230464 \\
\hline 57 & $\mathrm{C}$ & -3.56552 & -3.047752 & -0.69043 \\
\hline 58 & $\mathrm{H}$ & -4.636614 & -3.173918 & -0.72966 \\
\hline
\end{tabular}




$\begin{array}{rlrrr}59 & \mathrm{C} & -3.480569 & -0.524957 & -0.498351 \\ 6 \mathrm{O} & \mathrm{C} & 3.454878 & -0.648882 & -0.514897 \\ 61 & \mathrm{C} & 7.753546 & -0.66303 & -0.764901 \\ 62 & \mathrm{H} & 8.836945 & -0.666133 & -0.828776 \\ 63 & \mathrm{C} & 0.148561 & 7.166771 & -0.140913 \\ 64 & \mathrm{H} & 0.176017 & 8.251317 & -0.111467 \\ 65 & \mathrm{C} & -0.850883 & -1.867546 & 4.103618 \\ 66 & \mathrm{H} & -1.526284 & -2.586806 & 4.552025 \\ 67 & \mathrm{C} & 0.862933 & -0.201446 & 4.245537 \\ 68 & \mathrm{H} & 1.556586 & 0.412394 & 4.808534 \\ 69 & \mathrm{C} & -0.839903 & -1.69596 & 2.72405 \\ 70 & \mathrm{H} & -1.497495 & -2.268516 & 2.080156 \\ 71 & \mathrm{C} & 0.017181 & -1.106229 & 4.879838 \\ 72 & \mathrm{H} & 0.035176 & -1.216948 & 5.959024 \\ 73 & \mathrm{H} & 0.86761 & 6.929978 & 1.875132 \\ 74 & \mathrm{H} & -0.575417 & 7.076511 & -2.167095 \\ 75 & \mathrm{H} & 7.713576 & -0.033792 & 1.29423 \\ 76 & \mathrm{H} & 7.467643 & -1.292865 & -2.804355 \\ 77 & \mathrm{H} & -7.695285 & 0.077635 & 1.41785 \\ 78 & \mathrm{H} & -7.541548 & -0.892585 & -2.763\end{array}$

Cartesian Coordinates of $\mathbf{9 Z n}(6-311 \mathrm{G}(\mathrm{d})(\mathrm{C}, \mathrm{H}, \mathrm{N}, \mathrm{O})-\mathrm{cc}-\mathrm{pVQZ}(\mathrm{Zn})$ Basis sets) UB3LYP (singlet): $E_{\text {total }}=-7072.12031989$ Hartree

$\begin{array}{rrrrr}1 & \mathrm{C} & -0.210209 & 1.274885 & 1.615418 \\ 2 & \mathrm{C} & 0.415412 & 2.524886 & 1.92431 \\ 3 & \mathrm{H} & 1.459544 & 2.652307 & 2.165264 \\ 4 & \mathrm{C} & -0.567104 & 3.471844 & 1.857573 \\ 5 & \mathrm{H} & -0.468498 & 4.533849 & 2.021208 \\ 6 & \mathrm{C} & -1.781273 & 2.791716 & 1.47659 \\ 7 & \mathrm{C} & -2.998148 & 3.411118 & 1.152566 \\ 8 & \mathrm{C} & -4.141533 & 2.76346 & 0.621014 \\ 9 & \mathrm{C} & -5.389271 & 3.418891 & 0.311457 \\ 10 & \mathrm{H} & -5.59999 & 4.467724 & 0.450743 \\ 11 & \mathrm{C} & -6.238424 & 2.460264 & -0.152476\end{array}$




\begin{tabular}{|c|c|c|c|c|}
\hline 12 & $\mathrm{H}$ & -7.264871 & 2.585554 & -0.460713 \\
\hline 13 & $\mathrm{C}$ & $-5 \cdot 522204$ & 1.20872 & -0.105628 \\
\hline 14 & $\mathrm{C}$ & -6.094435 & -0.052332 & -0.385852 \\
\hline 15 & $\mathrm{C}$ & -5.498853 & -1.303073 & -0.114362 \\
\hline 16 & $\mathrm{C}$ & -6.156245 & -2.580259 & -0.250287 \\
\hline 17 & $\mathrm{H}$ & -7.152446 & -2.73804 & -0.632872 \\
\hline 18 & $\mathrm{C}$ & -5.289529 & -3.522377 & 0.214942 \\
\hline 19 & $\mathrm{H}$ & -5.458601 & -4.585048 & 0.29237 \\
\hline 20 & $\mathrm{C}$ & -4.082923 & -2.831883 & 0.601336 \\
\hline 21 & $\mathrm{C}$ & -2.929936 & -3.457522 & 1.139026 \\
\hline 22 & $\mathrm{C}$ & -1.730416 & -2.81436 & 1.477793 \\
\hline 23 & $\mathrm{C}$ & -0.506294 & -3.471203 & 1.868902 \\
\hline 24 & $\mathrm{H}$ & -0.389726 & -4.530564 & 2.038141 \\
\hline 25 & $\mathrm{C}$ & 0.458475 & -2.506561 & 1.93737 \\
\hline 26 & $\mathrm{H}$ & 1.503625 & -2.614298 & 2.183492 \\
\hline 27 & $\mathrm{C}$ & -0.187881 & -1.269017 & 1.619714 \\
\hline 28 & $\mathrm{C}$ & 0.504047 & 0.009113 & 1.549433 \\
\hline 29 & $\mathrm{C}$ & -3.089171 & 4.888356 & 1.372537 \\
\hline 30 & $\mathrm{C}$ & -3.103752 & 5.410836 & 2.671706 \\
\hline 31 & $\mathrm{H}$ & -3.054998 & 4.732396 & 3.51688 \\
\hline 32 & $\mathrm{C}$ & -3.192127 & 6.784729 & 2.884056 \\
\hline 33 & $\mathrm{C}$ & -3.257147 & 7.658237 & 1.800494 \\
\hline 34 & $\mathrm{H}$ & -3.322465 & 8.72892 & 1.965564 \\
\hline 35 & $\mathrm{C}$ & -3.236204 & 7.149376 & 0.503293 \\
\hline 36 & $\mathrm{C}$ & -3.157363 & $5 \cdot 775132$ & 0.290761 \\
\hline 37 & $\mathrm{H}$ & -3.137165 & $5 \cdot 382943$ & -0.720464 \\
\hline 38 & $\mathrm{C}$ & -7.479001 & -0.052354 & -0.955217 \\
\hline 39 & $\mathrm{C}$ & -8.582969 & -0.422783 & -0.177319 \\
\hline 40 & $\mathrm{H}$ & -8.431005 & -0.713331 & 0.856926 \\
\hline 41 & $\mathrm{C}$ & -9.867943 & -0.406825 & -0.715222 \\
\hline 42 & $\mathrm{C}$ & -10.067918 & -0.026034 & -2.040699 \\
\hline 43 & $\mathrm{H}$ & -11.068759 & -0.016059 & -2.460334 \\
\hline 44 & $\mathrm{C}$ & -8.976334 & 0.343823 & -2.823607 \\
\hline 45 & $\mathrm{C}$ & -7.692186 & 0.335459 & -2.283709 \\
\hline
\end{tabular}




\begin{tabular}{|c|c|c|c|c|}
\hline 46 & $\mathrm{H}$ & -6.843415 & 0.623306 & -2.895264 \\
\hline 47 & $\mathrm{C}$ & -2.987042 & -4.939773 & 1.341969 \\
\hline 48 & $\mathrm{C}$ & -2.979253 & -5.8179 & 0.251375 \\
\hline 49 & $\mathrm{H}$ & -2.925712 & -5.416078 & -0.754902 \\
\hline 50 & $\mathrm{C}$ & -3.026363 & -7.195845 & 0.449535 \\
\hline 51 & $\mathrm{C}$ & -3.089385 & -7.716822 & 1.740545 \\
\hline 52 & $\mathrm{H}$ & -3.129065 & -8.790454 & 1.894235 \\
\hline 53 & $\mathrm{C}$ & -3.099188 & -6.851692 & 2.832618 \\
\hline 54 & $\mathrm{C}$ & -3.044036 & -5.473732 & 2.634957 \\
\hline 55 & $\mathrm{H}$ & -3.052465 & -4.80161 & 3.486594 \\
\hline 56 & $\mathrm{Zn}$ & -2.76743 & -0.020326 & 0.519532 \\
\hline 57 & $\mathrm{~N}$ & -1.526565 & 1.439749 & 1.366617 \\
\hline 58 & $\mathrm{~N}$ & -4.250724 & 1.42514 & 0.355196 \\
\hline 59 & $\mathrm{~N}$ & -4.235626 & -1.489453 & 0.384756 \\
\hline 60 & $\mathrm{~N}$ & -1.499425 & -1.458101 & 1.364903 \\
\hline 61 & $\mathrm{O}$ & 1.763241 & 0.019855 & 1.446324 \\
\hline 62 & $\mathrm{C}$ & 0.210203 & -1.274867 & -1.615419 \\
\hline 63 & $\mathrm{C}$ & -0.415421 & -2.524862 & -1.924324 \\
\hline 64 & $\mathrm{H}$ & -1.459552 & -2.652276 & -2.165285 \\
\hline 65 & $\mathrm{C}$ & 0.567091 & -3.471825 & -1.857593 \\
\hline 66 & $\mathrm{H}$ & 0.468481 & -4.533827 & -2.021239 \\
\hline 67 & $\mathrm{C}$ & 1.781262 & -2.791704 & -1.476606 \\
\hline 68 & $\mathrm{C}$ & 2.998137 & -3.411113 & -1.152592 \\
\hline 69 & $\mathrm{C}$ & 4.141523 & -2.763464 & -0.62103 \\
\hline 70 & $\mathrm{C}$ & $5 \cdot 389261$ & -3.4189 & -0.311481 \\
\hline 71 & $\mathrm{H}$ & 5.599979 & -4.467732 & -0.450781 \\
\hline 72 & $\mathrm{C}$ & 6.238413 & -2.460281 & 0.152469 \\
\hline 73 & $\mathrm{H}$ & 7.264859 & -2.585574 & 0.460709 \\
\hline 74 & $\mathrm{C}$ & $5 \cdot 522196$ & -1.208734 & 0.105629 \\
\hline 75 & $\mathrm{C}$ & 6.09443 & 0.052315 & 0.385864 \\
\hline 76 & $\mathrm{C}$ & 5.498854 & 1.30306 & 0.114381 \\
\hline 77 & $\mathrm{C}$ & 6.156253 & 2.580242 & 0.25031 \\
\hline 78 & $\mathrm{H}$ & 7.152455 & 2.738016 & 0.632896 \\
\hline 79 & $\mathrm{C}$ & 5.289542 & $3 \cdot 522366$ & -0.214917 \\
\hline
\end{tabular}




\begin{tabular}{|c|c|c|c|c|}
\hline 80 & $\mathrm{H}$ & $5 \cdot 458619$ & 4.585037 & -0.292342 \\
\hline 81 & $\mathrm{C}$ & 4.082934 & 2.83188 & -0.60132 \\
\hline 82 & $\mathrm{C}$ & 2.929951 & 3.457526 & -1.139012 \\
\hline 83 & $\mathrm{C}$ & 1.730429 & 2.81437 & -1.477785 \\
\hline 84 & $\mathrm{C}$ & 0.506312 & 3.47122 & -1.868896 \\
\hline 85 & $\mathrm{H}$ & 0.38975 & 4.530582 & -2.038134 \\
\hline 86 & $\mathrm{C}$ & -0.458461 & 2.506584 & -1.937371 \\
\hline 87 & $\mathrm{H}$ & -1.50361 & 2.614326 & -2.183495 \\
\hline 88 & $\mathrm{C}$ & 0.187887 & 1.269036 & -1.619713 \\
\hline 89 & $\mathrm{C}$ & -0.50405 & -0.009091 & -1.549432 \\
\hline 90 & $\mathrm{C}$ & 3.089157 & -4.888348 & -1.372584 \\
\hline 91 & $\mathrm{C}$ & 3.103722 & -5.410809 & -2.671761 \\
\hline 92 & $\mathrm{H}$ & 3.05496 & -4.732356 & -3.516924 \\
\hline 93 & $\mathrm{C}$ & 3.192095 & -6.784699 & -2.884132 \\
\hline 94 & $\mathrm{C}$ & 3.257127 & -7.658223 & -1.800585 \\
\hline 95 & $\mathrm{H}$ & $3 \cdot 322444$ & -8.728904 & -1.965671 \\
\hline 96 & $\mathrm{C}$ & 3.2362 & -7.149381 & -0.503376 \\
\hline 97 & $\mathrm{C}$ & 3.157363 & $-5 \cdot 77514$ & -0.290822 \\
\hline 98 & $\mathrm{H}$ & 3.137176 & $-5 \cdot 382967$ & 0.720409 \\
\hline 99 & $\mathrm{C}$ & 7.478995 & 0.052328 & 0.955235 \\
\hline 100 & $\mathrm{C}$ & 8.582965 & 0.422764 & 0.177343 \\
\hline 101 & $\mathrm{H}$ & 8.431004 & 0.713325 & -0.8569 \\
\hline 102 & $\mathrm{C}$ & 9.867938 & 0.406798 & 0.71525 \\
\hline 103 & $\mathrm{C}$ & 10.067908 & 0.025991 & 2.040723 \\
\hline 104 & $\mathrm{H}$ & 11.068748 & 0.01601 & 2.460361 \\
\hline 105 & $\mathrm{C}$ & 8.976321 & -0.343873 & 2.823624 \\
\hline 106 & $\mathrm{C}$ & 7.692175 & -0.335501 & 2.283722 \\
\hline 107 & $\mathrm{H}$ & 6.843401 & -0.623355 & 2.895272 \\
\hline 108 & $\mathrm{C}$ & 2.987065 & 4.939777 & -1.34195 \\
\hline 109 & $\mathrm{C}$ & 2.979275 & 5.817901 & -0.251353 \\
\hline 110 & $\mathrm{H}$ & 2.925727 & 5.416076 & 0.754922 \\
\hline 111 & $\mathrm{C}$ & 3.026394 & 7.195846 & -0.449509 \\
\hline 112 & $\mathrm{C}$ & 3.089426 & $7 \cdot 716826$ & -1.740518 \\
\hline 113 & $\mathrm{H}$ & 3.129114 & 8.790459 & -1.894204 \\
\hline
\end{tabular}




\begin{tabular}{|c|c|c|c|c|}
\hline 114 & $\mathrm{C}$ & 3.099231 & 6.8517 & -2.832594 \\
\hline 115 & $\mathrm{C}$ & 3.044069 & 5.47374 & -2.634937 \\
\hline 116 & $\mathrm{H}$ & 3.052499 & 4.80162 & -3.486576 \\
\hline 117 & $\mathrm{Zn}$ & 2.76742 & 0.020327 & -0.519501 \\
\hline 118 & $\mathrm{~N}$ & 1.526558 & -1.439737 & -1.366618 \\
\hline 119 & $\mathrm{~N}$ & 4.250715 & -1.425147 & -0.355196 \\
\hline 120 & $\mathrm{~N}$ & 4.235628 & 1.489447 & -0.384743 \\
\hline 121 & $\mathrm{~N}$ & 1.49943 & 1.458112 & -1.364897 \\
\hline 122 & $\mathrm{O}$ & -1.763241 & -0.019825 & -1.446324 \\
\hline 123 & $\mathrm{H}$ & 3.210938 & -7.171543 & -3.898082 \\
\hline 124 & $\mathrm{H}$ & 3.278942 & -7.823018 & 0.346647 \\
\hline 125 & $\mathrm{H}$ & -3.150456 & -7.24817 & 3.841727 \\
\hline 126 & $\mathrm{H}$ & -3.010982 & -7.862724 & -0.406733 \\
\hline 127 & $\mathrm{H}$ & 3.011012 & 7.862723 & 0.406761 \\
\hline 128 & $\mathrm{H}$ & 3.150506 & 7.248181 & -3.841701 \\
\hline 129 & $\mathrm{H}$ & 9.122825 & -0.639888 & 3.857557 \\
\hline 130 & $\mathrm{H}$ & 10.713446 & 0.689731 & 0.096179 \\
\hline 131 & $\mathrm{H}$ & -3.278935 & 7.823 & -0.34674 \\
\hline 132 & $\mathrm{H}$ & -3.210982 & 7.171588 & 3.897999 \\
\hline 133 & $\mathrm{H}$ & -10.713448 & -0.689753 & -0.096145 \\
\hline 134 & $\mathrm{H}$ & -9.122841 & 0.639826 & -3.857542 \\
\hline
\end{tabular}

Cartesian Coordinates of 1oNi (cation part only, 6-311G(d)(C,H,N,O)-cc-pVQZ(Ni) Basis sets) RB3LYP (singlet): $E_{\text {total }}=-3264.778642151$ Hartree

$\begin{array}{rlrrr}1 & \mathrm{C} & 1.165875 & 2.115253 & -0.135762 \\ 2 & \mathrm{C} & 2.789629 & -1.823341 & -0.165292 \\ 3 & \mathrm{C} & 4.785464 & -0.710456 & 0.753895 \\ 4 & \mathrm{C} & 3.334704 & 1.859473 & 0.375343 \\ 5 & \mathrm{C} & -5.739851 & -1.415483 & -0.025614 \\ 6 & \mathrm{C} & -2.697696 & 0.411372 & 0.225958 \\ 7 & \mathrm{C} & 6.406769 & -1.510484 & 2.368633 \\ 8 & \mathrm{C} & -2.65004 & -2.015175 & -0.108354 \\ 9 & \mathrm{C} & -0.08184 & 2.736011 & -0.304659 \\ 10 & \mathrm{C} & 7.427598 & -0.875476 & 1.665076\end{array}$




\begin{tabular}{|c|c|c|c|c|}
\hline 11 & $\mathrm{C}$ & 3.374283 & -0.658478 & 0.287497 \\
\hline 12 & $\mathrm{C}$ & 3.503091 & -3.023744 & -0.514249 \\
\hline 13 & $\mathrm{C}$ & -1.284814 & 2.023124 & -0.205036 \\
\hline 14 & $\mathrm{C}$ & -0.155305 & 4.206403 & -0.510669 \\
\hline 15 & $\mathrm{C}$ & 2.689991 & 0.591873 & 0.245731 \\
\hline 16 & $\mathrm{C}$ & 2.398269 & 2.807106 & 0.077358 \\
\hline 17 & $\mathrm{C}$ & -2.563193 & 2.635636 & -0.013802 \\
\hline 18 & $\mathrm{C}$ & -3.440391 & 1.630774 & 0.277382 \\
\hline 19 & $\mathrm{C}$ & -3.303167 & -0.880164 & 0.321413 \\
\hline 20 & $\mathrm{C}$ & -6.316198 & -0.608096 & 2.580253 \\
\hline 21 & $\mathrm{C}$ & 5.094133 & -1.441212 & 1.91052 \\
\hline 22 & $\mathrm{C}$ & -0.679717 & 4.705435 & -1.712204 \\
\hline 23 & $\mathrm{C}$ & -0.333404 & 6.967153 & -0.942483 \\
\hline 24 & $\mathrm{C}$ & -4.705803 & -0.953166 & 0.802065 \\
\hline 25 & $\mathrm{C}$ & -0.753893 & 6.077929 & -1.929609 \\
\hline 26 & $\mathrm{C}$ & 2.618279 & -3.839596 & -1.154006 \\
\hline 27 & $\mathrm{C}$ & 1.37093 & -3.150882 & -1.138877 \\
\hline 28 & $\mathrm{C}$ & -1.149606 & -3.255211 & -1.078106 \\
\hline 29 & $\mathrm{C}$ & -7.048229 & -1.464077 & 0.446411 \\
\hline 30 & $\mathrm{C}$ & 5.81869 & -0.07254 & 0.051951 \\
\hline 31 & $\mathrm{C}$ & -3.23817 & $-3 \cdot 313647$ & -0.310124 \\
\hline 32 & $\mathrm{C}$ & $-7 \cdot 337851$ & -1.069584 & 1.75101 \\
\hline 33 & $\mathrm{C}$ & 0.123759 & -3.77706 & -1.60263 \\
\hline 34 & $\mathrm{C}$ & -5.010758 & -0.534429 & 2.106473 \\
\hline 35 & $\mathrm{C}$ & 7.131745 & -0.164876 & 0.502155 \\
\hline 36 & $\mathrm{C}$ & -2.314209 & -4.070298 & -0.966441 \\
\hline 37 & $\mathrm{C}$ & 0.16807 & 6.479387 & 0.263369 \\
\hline 38 & $\mathrm{C}$ & 0.266759 & 5.108022 & 0.477394 \\
\hline 39 & $\mathrm{H}$ & 4.368645 & 2.015661 & 0.634522 \\
\hline 40 & $\mathrm{H}$ & -5.524087 & -1.701891 & -1.048918 \\
\hline 41 & $\mathrm{H}$ & 8.451361 & -0.938328 & 2.017216 \\
\hline 42 & $\mathrm{H}$ & 4.554038 & -3.190956 & -0.33864 \\
\hline 43 & $\mathrm{H}$ & 2.535792 & 3.874781 & 0.029801 \\
\hline 44 & $\mathrm{H}$ & -2.759428 & 3.695022 & -0.053498 \\
\hline
\end{tabular}




\begin{tabular}{|c|c|c|c|c|}
\hline 45 & $\mathrm{H}$ & -4.495177 & 1.712011 & 0.48426 \\
\hline 46 & $\mathrm{H}$ & 4.301177 & -1.936886 & 2.460133 \\
\hline 47 & $\mathrm{H}$ & -1.007034 & 4.01623 & -2.483186 \\
\hline 48 & $\mathrm{H}$ & -0.40084 & 8.036517 & -1.109785 \\
\hline 49 & $\mathrm{H}$ & 2.776217 & -4.814557 & -1.587938 \\
\hline 50 & $\mathrm{H}$ & $5 \cdot 599858$ & 0.464363 & -0.864584 \\
\hline 51 & $\mathrm{H}$ & -4.231934 & -3.600353 & -0.006342 \\
\hline $5^{2}$ & $\mathrm{H}$ & -4.219447 & -0.175413 & 2.755815 \\
\hline 53 & $\mathrm{H}$ & -2.387184 & -5.088671 & -1.31482 \\
\hline 54 & $\mathrm{H}$ & 0.63958 & 4.734573 & 1.424884 \\
\hline 55 & $\mathrm{~N}$ & -1.322585 & -2.033244 & -0.564905 \\
\hline 56 & $\mathrm{~N}$ & 1.451908 & -1.947214 & $-0.5575^{21}$ \\
\hline 57 & $\mathrm{~N}$ & 1.363898 & 0.751497 & -0.045636 \\
\hline 58 & $\mathrm{~N}$ & -1.388832 & 0.653681 & -0.06907 \\
\hline 59 & $\mathrm{Ni}$ & 0.027322 & -0.638824 & -0.31692 \\
\hline 60 & $\mathrm{O}$ & 0.145129 & -4.774526 & -2.298765 \\
\hline 61 & $\mathrm{H}$ & 7.92595 & 0.314563 & -0.059638 \\
\hline 62 & $\mathrm{H}$ & 6.630769 & -2.065164 & 3.273208 \\
\hline 63 & $\mathrm{H}$ & -1.143183 & 6.451829 & -2.870246 \\
\hline 64 & $\mathrm{H}$ & 0.479813 & 7.167411 & 1.041635 \\
\hline 65 & $\mathrm{H}$ & -7.842034 & -1.806873 & -0.208171 \\
\hline 66 & $\mathrm{H}$ & -8.35697 & -1.115618 & 2.118888 \\
\hline 67 & $\mathrm{H}$ & -6.536058 & -0.302867 & 3.5974 \\
\hline
\end{tabular}

Cartesian Coordinates of $\mathbf{1 0 Z n}$ (cation part only, 6-311G(d)(C,H,N,O)-cc-pVQZ(Zn) Basis sets) RB3LYP (singlet): $E_{\text {total }}=-3535.83126679$ Hartree

$\begin{array}{lllrr}1 & \mathrm{~N} & 1.459793 & 0.738011 & -0.001012 \\ 2 & \mathrm{~N} & -1.46501 & -2.181419 & -0.055106 \\ 3 & \mathrm{~N} & 1.483453 & -2.168659 & -0.03659 \\ 4 & \mathrm{O} & 0.025123 & -5.42835 & -0.088187 \\ 5 & \mathrm{C} & 2.867205 & -1.954282 & 0.002421 \\ 6 & \mathrm{~N} & -1.467223 & 0.727094 & 0.072904 \\ 7 & \mathrm{C} & 2.810636 & 0.55448 & 0.056902 \\ 8 & \mathrm{C} & 1.248776 & 2.100549 & 0.007617\end{array}$




\begin{tabular}{|c|c|c|c|c|}
\hline 9 & $\mathrm{C}$ & 1.320098 & -3.495428 & -0.048904 \\
\hline 10 & $\mathrm{C}$ & 2.508421 & 2.781034 & 0.085603 \\
\hline 11 & $\mathrm{H}$ & 2.646937 & 3.848783 & 0.135862 \\
\hline 12 & $\mathrm{C}$ & -4.968151 & -0.72326 & 0.039088 \\
\hline 13 & $\mathrm{C}$ & 4.976839 & -0.6861 & 0.076585 \\
\hline 14 & $\mathrm{C}$ & 3.479663 & 1.819725 & 0.098335 \\
\hline 15 & $\mathrm{H}$ & 4.546384 & 1.969632 & 0.130332 \\
\hline 16 & $\mathrm{C}$ & -3.48118 & -0.744576 & 0.023621 \\
\hline 17 & $\mathrm{C}$ & -1.285112 & -3.506541 & -0.008398 \\
\hline 18 & $\mathrm{C}$ & -1.264375 & 2.086539 & -0.043513 \\
\hline 19 & $\mathrm{C}$ & 3.486082 & -0.713825 & 0.047571 \\
\hline 20 & $\mathrm{C}$ & -0.010916 & 2.739738 & -0.036417 \\
\hline 21 & $\mathrm{C}$ & -2.81387 & 0.528459 & -0.01653 \\
\hline 22 & $\mathrm{C}$ & -0.04459 & 7.026721 & -0.171596 \\
\hline 23 & $\mathrm{H}$ & -0.053513 & 8.110657 & -0.205032 \\
\hline 24 & $\mathrm{C}$ & -2.848366 & -1.980715 & 0.028843 \\
\hline 25 & $\mathrm{C}$ & $5 \cdot 712272$ & -1.128711 & -1.031523 \\
\hline 26 & $\mathrm{H}$ & 5.192709 & -1.475583 & -1.918261 \\
\hline 27 & $\mathrm{C}$ & $7 \cdot 775745$ & -0.649776 & 0.126645 \\
\hline 28 & $\mathrm{H}$ & 8.859937 & -0.636052 & 0.146897 \\
\hline 29 & $\mathrm{C}$ & -0.612 & 4.966069 & 0.950917 \\
\hline 30 & $\mathrm{H}$ & -1.04732 & 4.44902 & 1.799083 \\
\hline 31 & $\mathrm{C}$ & 5.659846 & -0.223796 & 1.210407 \\
\hline 32 & $\mathrm{H}$ & 5.101641 & 0.107202 & 2.07973 \\
\hline 33 & $\mathrm{C}$ & -0.610131 & 6.356743 & 0.912135 \\
\hline 34 & $\mathrm{C}$ & -0.021079 & 4.226878 & -0.085299 \\
\hline 35 & $\mathrm{C}$ & -5.699076 & -1.316283 & -1.001576 \\
\hline 36 & $\mathrm{H}$ & -5.174545 & -1.773368 & -1.833442 \\
\hline 37 & $\mathrm{C}$ & 0.531828 & 6.300704 & -1.21276 \\
\hline 38 & $\mathrm{C}$ & 0.019801 & -4.210024 & -0.058389 \\
\hline 39 & $\mathrm{C}$ & -2.523529 & 2.746367 & -0.229901 \\
\hline 40 & $\mathrm{H}$ & -2.66428 & 3.803244 & -0.388452 \\
\hline 41 & $\mathrm{C}$ & 7.10405 & -1.099861 & -1.007818 \\
\hline 42 & $\mathrm{C}$ & -7.050386 & -0.108095 & 1.116151 \\
\hline
\end{tabular}




\begin{tabular}{|c|c|c|c|c|}
\hline 43 & $\mathrm{C}$ & -7.768438 & -0.691486 & 0.072521 \\
\hline 44 & $\mathrm{H}$ & -8.852767 & -0.679243 & 0.086218 \\
\hline 45 & $\mathrm{C}$ & 7.050954 & -0.217861 & 1.236826 \\
\hline 46 & $\mathrm{C}$ & 0.556679 & 4.910484 & -1.166201 \\
\hline 47 & $\mathrm{H}$ & 0.999352 & 4.349906 & -1.982314 \\
\hline 48 & $\mathrm{C}$ & -3.486944 & 1.777073 & -0.210762 \\
\hline 49 & $\mathrm{H}$ & -4.547897 & 1.90752 & -0.348648 \\
\hline 50 & $\mathrm{C}$ & -5.65988 & -0.109462 & 1.094832 \\
\hline 51 & $\mathrm{H}$ & -5.106587 & 0.341181 & 1.911689 \\
\hline 52 & $\mathrm{C}$ & 2.567021 & -4.193728 & -0.017757 \\
\hline 53 & $\mathrm{H}$ & 2.674194 & -5.266852 & -0.011952 \\
\hline 54 & $\mathrm{C}$ & -7.090411 & -1.288531 & -0.988278 \\
\hline 55 & $\mathrm{C}$ & -3.497192 & -3.264019 & 0.14596 \\
\hline 56 & $\mathrm{H}$ & -4.55781 & -3.419209 & 0.262724 \\
\hline 57 & $\mathrm{C}$ & 3.534375 & -3.234133 & 0.027624 \\
\hline 58 & $\mathrm{H}$ & 4.601059 & -3.380394 & 0.087703 \\
\hline 59 & $\mathrm{C}$ & -2.51984 & -4.214651 & 0.111402 \\
\hline 60 & $\mathrm{H}$ & -2.613782 & -5.287248 & 0.173517 \\
\hline 61 & $\mathrm{H}$ & 0.962918 & 6.817024 & -2.063504 \\
\hline 62 & $\mathrm{H}$ & -1.051131 & 6.917215 & 1.729201 \\
\hline 63 & $\mathrm{H}$ & $7 \cdot 569108$ & 0.122953 & 2.126559 \\
\hline 64 & $\mathrm{H}$ & 7.662332 & -1.431407 & -1.876502 \\
\hline 65 & $\mathrm{H}$ & $-7 \cdot 574221$ & 0.34868 & 1.948696 \\
\hline 66 & $\mathrm{H}$ & -7.644041 & -1.734171 & -1.807429 \\
\hline 67 & $\mathrm{Zn}$ & 0.002143 & -0.712879 & -0.005228 \\
\hline
\end{tabular}

